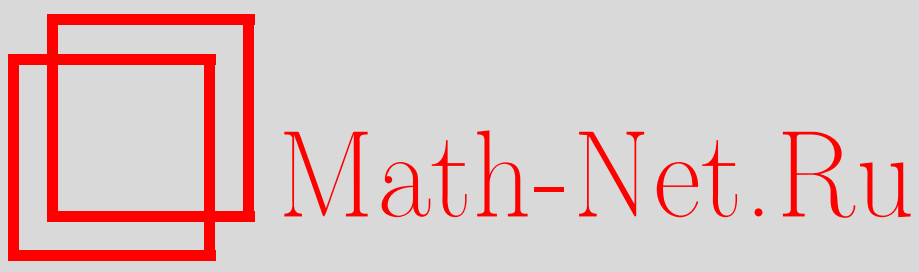

Е. М. Матвеев, Явная нижняя оценка однородной рациональной линейной формы от логарифмов алгебраических чисел. II, Изв. РАН. Сер. матем., 2000, том 64, выпуск $6,125-180$

DOI: https://doi.org/10.4213/im314

Использование Общероссийского математического портала Math-Net.Ru подразумевает, что вы прочитали и согласны с пользовательским соглашением

http: //www. mathnet.ru/rus/agreement

Параметры загрузки:

IP : 35.174 .16 .151

26 апреля 2023 г., 14:12:16 
УДК 511

Е. М. Матвеев

\title{
Явная нижняя оценка однородной рациональной линейной формы от логарифмов алгебраических чисел. II
}

\author{
В работе доказана показательная по количеству слагаемых оценка модуля \\ указанной в заглавии линейной формы. \\ Библиография: 23 наименования.
}

\section{§1. Введение}

В данной работе получено сушественное усиление результатов первой части (см. [1]). Изложение практически независимо от первой части работы, более того, некоторым темам, обсуждающимся и в [1], дан более развернутый комментарий.

Как и в [1], мы будем рассматривать только однородный рациональный случай линейных форм от логарифмов алгебраических чисел, т.е. выражения вида

$$
\Lambda=b_{1} \ln \alpha_{1}+\cdots+b_{n} \ln \alpha_{n},
$$

где $b_{1}, \ldots, b_{n} \in \mathbb{Z}$, а $\alpha_{1}, \ldots, \alpha_{n} \in \mathbb{K}^{*}$ - ненулевые числа алгебраического поля $\mathbb{K} \subseteq \mathbb{C}$ с некоторыми ненулевыми фиксированными значениями $\ln \alpha_{j}$.

Теория линейных форм от логарифмов имеет достаточно длинную историю. В последнее время здесь был достигнут ряд существенных успехов. Практически установилась зависимость оценки от $A_{j}$ - логарифмических высот чисел $\alpha_{j}$, от $\left|b_{j}\right|$, от степени поля $\mathbb{K}$ (более подробно об обозначениях будет сказано в следующих параграфах). Все последние опубликованные оценки вполне явные, позволяющие получать многочисленные приложения к другим задачам теории чисел.

Таким образом, интерес сместился к уточнению формы вхождения $n$ в оценку $\Lambda$. До недавнего времени главный член здесь имел вид $n^{2 n}$ (впервые это было сделано в [2]). При этом было замечено, что в случае так называемой сильной независимости чисел $\alpha_{1}, \ldots, \alpha_{n}$ можно получать оценки с главным членом $n^{n}$, и еше один множитель $n$ ! появляется при переходе к общему случаю. В данной работе под условием сильной независимости мы понимаем следующее равенство:

$$
\left[\mathbb{K}\left(\sqrt{\alpha_{1}}, \ldots, \sqrt{\alpha_{n}}\right): \mathbb{K}\right]=2^{n} .
$$

Полезное уточнение можно сделать для случая сильной независимости чисел $\alpha_{j}$ относительно параметра, обычно обозначаемого через $B$. Здесь можно считать (cM. [3])

$$
B=\max \left\{1, \max \left\{\left|b_{j}\right| A_{j} / A_{n}: 1 \leqslant j \leqslant n\right\}\right\},
$$

Работа выполнена при поддержке ИНТАС-РФФИ (грант № IR 97-1904).

(C) E. M. Матвеeв, 2000 
в то время как в обшем случае

$$
B^{*}=\max \left\{\left|b_{1}\right|, \ldots,\left|b_{n}\right|\right\} .
$$

Для растушего $A_{n}$ формула (1.3) дает заметное усиление оценки $\Lambda$. В ряде работ вводятся различные модификации выражения (1.3).

В работе [1] для случая сильной независимости была получена оценка без множителя $n^{n}$, а общий случай не рассматривался.

Итак, основными результатами данной статьи являются следующие.

1. Оценка линейной формы не содержит множитель $n^{n}$ во всех случаях, т.е. (1.2) устранено без ухудшения точности. В этом новизна статьи. Интересно, что ранее общий случай давал ухудшение оценки линейной формы при сведении его к случаю сильной независимости. В данной же работе получилось, что имеет место противоположная ситуация, а именно случай выполнения условия (1.2) является в некотором смысле наихудшим.

2. Из оценки $\Lambda$ удален множитель типа $\ln \left(A_{1} \ldots A_{n-1}\right)$, которьй присутствовал в работе [1]. Это достигнуто средствами, аналогичными приему работы [4].

3. Так же, как и в работе [1], в основной теореме отсутствуют индивидуальные нижние ограничения на числа $A_{j}$. Только в той работе условия теоремы включали нижнее ограничение на $\Omega$ - произведение чисел $A_{j}$. Теперь же имеется работа [5], где доказана приемлемая нижняя оценка для $\Omega$, что позволило снять указанное ограничение.

4. Так же, как и в [1], [3], под числом $B$ понимается (1.3), и этот параметр сохраняется для общего случая.

Выше указаны усиления предшествуюшей статьи. Вместе с тем данная работа содержит и некоторые ухудшения, вызванные стремлением автора сделать более прозрачным основной результат и уменьшить громоздкость изложения. Отличиями в худшую сторону от [1] являются следующие.

5. Из формулировок удален ряд параметров, позволяющих получать более точные оценки в частных случаях. Оставлен только минимальный набор основных параметров.

6. Упрошена схема арифметико-аналитического продолжения, но за счет уменьшения точности получаемых констант. Так что в случае выполнения условия (1.2) зависимость от $n$ в [1] лучше, чем в данной статье. Все же полученные константы вполне явные и лучше приведенных ранее (например, в [4]).

После устранения множителя $n^{n}$ в линейной форме $\Lambda$ основной зависимостью от $n$ становится показательная величина $C^{n}$, и вряд ли стоит рассчитывать, что такая зависимость может быть удалена. Так что зависимость полученных оценок от учтенных параметров, по-видимому, окончательная. В то же время, как будет видно из доказательства, появляются новые возможности усиления результатов.

\section{§2. Формулировка результатов}

На протяжении всей статьи мы сохраняем следующие обозначения: $\mathbb{K}$ - алгебраическое поле степени $D$ над $\mathbb{Q}$, вложенное в $\mathbb{C}$. Если $\mathbb{K} \subseteq \mathbb{R}$, то положим $\varkappa=1$, иначе $\varkappa=2$. Даны числа $\alpha_{1}, \ldots, \alpha_{n} \in \mathbb{K}^{*}$, имеющие абсолютные логарифмичес- 
кие высоты $\mathrm{h}\left(\alpha_{j}\right), 1 \leqslant j \leqslant n$ (определение см. в $\left.\S 5\right), \ln \alpha_{1}, \ldots, \ln \alpha_{n}-$ произвольные фиксированные ненулевые значения логарифмов. Пусть

$$
A_{j} \geqslant \max \left\{D \mathrm{~h}\left(\alpha_{j}\right),\left|\ln \alpha_{j}\right|\right\}, \quad 1 \leqslant j \leqslant n .
$$

В частности, допускается $\alpha_{j}=1$, где $\mathrm{h}\left(\alpha_{j}\right)=0$.

Рассматриваются линейная форма (1.1) с $b_{1}, \ldots, b_{n} \in \mathbb{Z}$ и параметром $B$ из (1.3). Положим для краткости

$$
\begin{gathered}
\Omega=A_{1} \ldots A_{n}, \\
C(n)=C(n, \varkappa)=\frac{16}{n ! \varkappa} e^{n}(2 n+1+2 \varkappa)(n+2)(4(n+1))^{n+1}\left(\frac{1}{2} e n\right)^{\varkappa}, \\
C_{0}=\ln \left(e^{4.4 n+7} n^{5.5} D^{2} \ln (e D)\right), \quad W_{0}=\ln (1.5 e B D \ln (e D)) .
\end{gathered}
$$

ТЕорема 2.1 (основная). Если $\ln \alpha_{1}, \ldots, \ln \alpha_{n}$ линейно независимы над $\mathbb{Z} u$ $b_{n} \neq 0, m o$

$$
\ln |\Lambda|>-C(n) C_{0} W_{0} D^{2} \Omega .
$$

Теорема 2.2. Пусть при некотором $r(1 \leqslant r \leqslant n) \ln \alpha_{1}, \ldots, \ln \alpha_{r}$ линейно независимы над $\mathbb{Z} ;$ при $j \leqslant r$ имеем (2.1), при $j>r$ положим

$$
A_{j} \geqslant \max \left\{D \mathrm{~h}\left(\alpha_{j}\right),\left|\ln \alpha_{j}\right|, 0.16\right\},
$$

если $b_{n}=0$, то для $j=n$ также положим (2.4).

Тогда если $\Lambda \neq 0$, mo

$$
\ln |\Lambda|>-C(n) \max \{1, n / 6\} C_{0} W_{0} D^{2} \Omega \text {. }
$$

СЛЕДСТВИЕ 2.3. Если $\Lambda \neq 0, A_{j}$ из (2.4), $B$ из (1.3), mo

$$
\begin{aligned}
\ln |\Lambda| & >-C_{1}(n) D^{2} \Omega \ln (e D) \ln (e B), \\
C_{1}(n)=C_{1}(n, \varkappa) & =\min \left\{\frac{1}{\varkappa}\left(\frac{1}{2} e n\right)^{\varkappa} 30^{n+3} n^{3.5}, 2^{6 n+20}\right\},
\end{aligned}
$$

при этом $B$ мохсет быть заменено на $B^{*}$ из (1.4).

Замечание 2.1. Мы включили множитель $D$ в определение $A_{j}$. Обычно его выносят за скобки, придавая выражению сходство с абсолютной высотой, а множитель $D$ появляется в оценке линейной формы $\Lambda$. Но поскольку при вынесении $D$ за скобки зависимость в $A_{j}$ от $D$ появляется в виде $\frac{1}{D}\left|\ln \alpha_{j}\right|$, то не видно большого смысла в выделении $D$, и во всех выкладках мы реально имеем дело с $A_{j}$ вида (2.1). 
ЗАмечаниЕ 2.2. Фиксированная нижняя гранища в (2.4) для $A_{j}$ достаточна для всех известных алгебраических чисел, кроме корней из 1 , которые могут давать малое значение величины $A$. Также отметим, что при отсутствии информации о линейной независимости логарифмов нижние границы для $A_{j}$ необходимы. Иначе, например, расшепление компоненты $b_{j} \ln \alpha_{j}$ с мальм $A_{j}$ на несколько слагаемых может привести к неверному усилению оценки.

Теорема 2.2 оставляет возможность некоторым из значений $A_{j}$ быть малыми. Более того, если в поле есть корень из единицы $\alpha_{0}$ высокой степени $\left(\alpha_{0} \neq \alpha_{j}\right.$, $j \geqslant 1$ ), то его можно формально добавить к линейной форме с $b_{0}=0$. Это только улучшит оценку линейной формы. Эффект усиления оценок для полей, содержащих корни из 1, в свое время обнаружил Фельдман (см. [6]).

ЗАмЕчАниЕ 2.3. Следствие 2.3 приведено для более простого применения результатов статьи в грубых случаях и для облегчения сравнения с предшествующими работами. Для $n=2$ известны сушественно более хорошие оценки.

\section{§ 3. Некоторые обозначения и соглашения}

Мы придерживаемся векторных обозначений, введенных в работе [1]. Далее будут встречаться векторы с различными способами нумерации координат. Для некоторых из них зафиксируем обозначения:

1) $\bar{u}=\left(u_{1}, \ldots, u_{n}\right)-$ с помошью знака $^{-}$;

2) $\tilde{u}=\left(u_{1}, \ldots, u_{n-1}\right)-$ с помощью знака ;

3) $\bar{u}^{\prime}=\left(u_{0}, \ldots, u_{n}\right)-$ с помошью знаков $^{-\prime}$;

4) $\tilde{u}^{\prime}=\left(u_{0}, \ldots, u_{n-1}\right)-$ с помошью знаков ${ }^{\prime \prime}$.

В случаях, когда нумерация координат иная или не имеет значения, будем обозначать векторы жирным шрифтом, объясняя при необходимости способ нумерации (как правило, будут использоваться индексы $j=1, \ldots, J)$. Координаты векторов будут обозначаться той же буквой, что и сам вектор.

Для действительных векторов $\mathbf{u}, \mathbf{v}$ условие $\mathbf{u} \geqslant \mathbf{v}$ (или $\mathbf{u}>\mathbf{v}$ ) означает соответствующее неравенство для каждой координаты.

Если у нас есть скалярная функция $f(x)$ одной скалярной переменной (она может зависеть от других числовых аргументов), то мы определим вектор $f(\mathbf{u})$ для $\mathbf{u}=\left(u_{1}, \ldots, u_{J}\right)$ следуюшим образом:

$$
f(\mathbf{u})=\left(f\left(u_{1}\right), \ldots, f\left(u_{J}\right)\right) ;
$$

например,

$$
\ln \bar{\alpha}=\left(\ln \alpha_{1}, \ldots, \ln \alpha_{n}\right), \quad \widetilde{m} !=\left(m_{1} !, \ldots, m_{n-1} !\right) .
$$

Для скалярной функции $f(x, y)$ и векторов $\mathbf{u}, \mathbf{v}$ положим

$$
f(\mathbf{u}, \mathbf{v})=\prod_{j=1}^{J} f\left(u_{j}, v_{j}\right) ;
$$

например,

$$
\bar{\alpha}^{\bar{l}}=\prod_{j=1}^{n} \alpha_{j}^{l_{j}}
$$


Будем здесь говорить, что $\bar{l}-$ мультистепень $\bar{\alpha}$.

Если мы хотим получить не вектор $f(\mathbf{u})$, а произведение соответствующих элементов, то следует писать

$$
f(\mathbf{u})^{\mathbf{1}}=\prod_{j=1}^{J} f\left(u_{j}\right)^{1},
$$

вводя вектор $\mathbf{1}=(1, \ldots, 1)$. Будем считать $0^{0}=1$.

Версии следующей очевидной леммы традиционно используются в схеме Бейкера.

ЛЕмма 3.1. Пусть даны наборы многочленов $f(z, m), g(z, m)$ (no $z)$; $\operatorname{deg}_{z} f=\operatorname{deg}_{z} g=m, \quad m=0,1, \ldots \quad$ Тогда многочлены $f(\mathbf{z}, \mathbf{m})$ линейно выражаются через $g(\mathbf{z}, \mathbf{t}), \mathbf{t} \leqslant \mathbf{m}$, и наоборот.

Следуя современным обозначениям (см. [4]), введем для функций $f\left(\widetilde{Y^{\prime}}\right)=$ $f\left(Y_{0}, \ldots, Y_{n}\right)$ операторы дифференцирования

$$
\partial_{0}=\frac{\partial}{\partial Y_{0}}, \quad \partial_{j}=b_{n} Y_{j} \frac{\partial}{\partial Y_{j}}-b_{j} Y_{n} \frac{\partial}{\partial Y_{n}}, \quad 1 \leqslant j \leqslant n,
$$

где $b_{j}$ - те же, что и в линейной форме $\Lambda$ (см. (1.1)). Производные высших порядков можно записьвать с помошью мультистепеней, например

$$
\tilde{\partial}^{\widetilde{m}}=\partial_{1}^{m_{1}} \ldots \partial_{n-1}^{m_{n-1}} .
$$

С указанными операторами связано следующее обозначение:

$$
\tilde{\chi}(\bar{u})=\left(\chi_{1}, \ldots, \chi_{n-1}\right), \quad \chi_{j}=u_{j} b_{n}-u_{n} b_{j}, \quad 1 \leqslant j \leqslant n-1,
$$

где обязательно $\bar{u}$ - вектор первого типа и при этом получается, что $\tilde{\chi}-$ вектор второго типа. Связь задается формулой

$$
\tilde{\partial}^{\widetilde{m}}\left(\bar{Y}^{\bar{u}}\right)=(\tilde{\chi}(\bar{u}))^{\widetilde{m}} \bar{Y}^{\bar{u}} \text {. }
$$

Множество натуральных (целых положительных) чисел будем обозначать через $\mathbb{N}$, а множество неотрицательных целых чисел - через $\mathbb{N}_{0} ;|\mathscr{L}|$ - количество элементов во множестве $\mathscr{L}$.

До $\S 21$ статья посвящена доказательству теоремы 2.1. В ее формулировку числа $A_{1}, \ldots, A_{n-1}$ входят симметрично, поэтому без ограничения общности будем считать, что $A_{1} \leqslant \cdots \leqslant A_{n-1}$.

В случае $\varkappa=1$ мы имеем $|\alpha|= \pm \alpha \in \mathbb{K}$, поэтому, положив $\alpha_{j}^{\prime}=\left|\alpha_{j}\right|$ и взяв действительное значение $\ln \alpha_{j}^{\prime}, A_{j}^{\prime}=A_{j}$, имеем

$$
|\Lambda| \geqslant\left|\Lambda^{\prime}\right|=\left|b_{1} \ln \alpha_{1}^{\prime}+\cdots+b_{n} \ln \alpha_{n}^{\prime}\right|,
$$

и поэтому здесь можно считать $\alpha_{j}>0$ и $\ln \alpha_{j} \in \mathbb{R}$. Дополнительно следует рассмотреть случай $\alpha_{j}= \pm 1$ с ненулевым значением $\ln \alpha_{j}$, поскольку здесь $\ln \alpha_{j}^{\prime}=0$, что не предусмотрено условиями теоремы 2.1. Но с учетом того, что здесь $A_{j} \geqslant \pi$, соответствуюшее слагаемое можно просто отбросить с улучшением оценки, а случай $\alpha_{n}= \pm 1$ рассматривается аналогично случаю $b_{n}=0$ в доказательстве теоремы 2.2.

В дальнейшем у нас будут встречаться выражения, зависящие от ранее введенных параметров. Для краткости мы будем при необходимости указывать только те из них, которые нужны в данный момент, например, писать $C_{0}, C_{0}(n)$ или $C_{0}(n, D)$. 


\section{§4. Решетки}

Приведем необходимые факты из основ геометрии чисел (см., например, [7] и [8]).

Пусть дано векторное пространство $\mathscr{V}$ над $\mathbb{R}$ с нормой $\|\cdot\|$. Тогда решеткой называется дискретная подгруппа $\mathrm{M} \subseteq \mathscr{V}$ (дискретность понимается в том смысле, что для любого $R$ условию $\|\mathbf{v}\| \leqslant R$ удовлетворяет только конечное число векторов $\mathbf{v} \in \mathrm{M})$. У решетки есть базис, через него целочисленно выражаются все векторы решетки. Количество векторов в базисе называется рангом решетки $(\operatorname{rank} \mathrm{M}) ;$ ввиду дискретности имеем $\operatorname{rank}_{\mathbb{Z}} \mathrm{M}=\operatorname{rank}_{\mathbb{R}} \mathrm{M}$.

Для набора векторов $\mathscr{U}$ и для множества $\mathscr{B} \subseteq \mathbb{R}$ через $\langle\mathscr{U}\rangle_{\mathscr{B}}$ мы будем обозначать множество линейных комбинаций (с конечным числом слагаемых) векторов из $\mathscr{U}$ с коэффициентами из $\mathscr{B}$. Чтобы конечно порожденная аддитивная подгруппа $\mathrm{M} \mathrm{являлась} \mathrm{решеткой,} \mathrm{должно} \mathrm{быть} \operatorname{rank} \mathrm{M}=\operatorname{dim}\langle\mathrm{M}\rangle_{\mathbb{R}}$. Если $\langle\mathrm{M}\rangle_{\mathbb{R}}=\mathscr{V}$, то говорят, что решетка полная. В [7] изучаются только полные решетки, но очевидно, что любая решетка $\mathrm{M}$ полна в $\langle\mathrm{M}\rangle_{\mathbb{R}}$.

Для заданной нормы $\|\cdot\|$ через $\mathscr{W}(\|\cdot\|)$ будем обозначать единичный шар нормы, т.е. множество векторов $\|\mathbf{v}\| \leqslant 1$. Число $\lambda_{k}$ является $k$-м последовательнылм минимумом множества М относительно нормирования $\|\cdot\|$, если это точная нижняя грань тех $\lambda>0$, для которых существуют $k$ линейно независимых векторов из $\lambda \mathscr{W}(\|\cdot\|) \cap \mathrm{M}$. У решетки ввиду ее дискретности сушествуют ненулевые последовательные минимумы, достигающиеся на некоторых векторах. Последовательные минимумы не убывают, и их число равно rank M.

Если пространство евклидово, то скалярное произведение в нем задает $m$-мерные объемы, индуцированные формулой

$$
\operatorname{Vol}_{m}\left\langle\mathbf{v}_{1}, \ldots, \mathbf{v}_{m}\right\rangle_{[0 ; 1)}=\left(\operatorname{det}\left(\mathbf{v}_{i}, \mathbf{v}_{j}\right)_{i, j=1, \ldots, m}\right)^{1 / 2},
$$

где $\left\langle\mathbf{v}_{1}, \ldots, \mathbf{v}_{m}\right\rangle_{[0 ; 1)}$ - параллелепипед, построенный на линейно независимых век$\operatorname{Topax} \mathbf{v}_{j}$.

Отметим, что наряду с евклидовой нормой в $\mathscr{V}$ можно одновременно работать и с другими нормами. Кроме того, евклидову метрику, задаюшую объемы, также можно задавать различными способами. Обычно в координатном пространстве по умолчанию берется стандартная метрика, но далее мы будем пользоваться и иными евклидовыми нормами. Если в какой-либо системе координат матрица евклидовой нормы равна $Q, V$ - матриша строк координат векторов $\mathbf{v}_{1}, \ldots, \mathbf{v}_{m}$, то

$$
\operatorname{Vol}_{m}\left\langle\mathbf{v}_{1}, \ldots, \mathbf{v}_{m}\right\rangle_{[0 ; 1)}=\left(\operatorname{det}\left(V Q V^{T}\right)\right)^{1 / 2} .
$$

Тело $\Pi \subseteq\langle\mathrm{M}\rangle_{\mathbb{R}}$ назьвают фундаментальной областью решетки $\mathrm{M}$, если множества $\Pi+\mathbf{v}, \mathbf{v} \in \mathrm{M}$, дают разбиение пространства $\langle\mathrm{M}\rangle_{\mathbb{R}}$. Так, П(М) $=\left\langle\mathbf{e}_{1}, \ldots\right.$ $\left.\ldots, \mathbf{e}_{n}\right\rangle_{[0 ; 1)}$, где $\mathbf{e}_{1}, \ldots, \mathbf{e}_{n}-$ базис решетки M, называют фундаментальным naраллелепипедом решетки. Все фундаментальные области имеют одинаковьй объем, называемый объемом решетки $\left(\operatorname{Vol}_{n} \mathrm{M}=\operatorname{Vol}_{n} \Pi(\mathrm{M})\right)$.

ПРЕДЛОЖЕНИЕ 4.1 (теорема Минковского о последовательных минимумах [7, гл. 8, теорема 5]). Пусть в евклидовом пространстве даны норма $\|\cdot\|$ и решетка $\mathrm{M}_{n}$ ранга $n$; ее последовательные минимумы $\lambda_{1}, \ldots, \lambda_{n}$ достигаются 
на векторах $\mathbf{v}_{1}, \ldots, \mathbf{v}_{n}: \mathrm{M}_{n}^{\prime}=\left\langle\mathbf{v}_{1}, \ldots, \mathbf{v}_{n}\right\rangle_{\mathbb{Z}} ;\left[\mathrm{M}_{n}: \mathrm{M}_{n}^{\prime}\right]-$ индекс $\mathrm{M}_{n}$ относительно $\mathrm{M}_{n}^{\prime}$. Тогда

$$
\frac{1}{n !} 2^{n}\left[\mathrm{M}_{n}: \mathrm{M}_{n}^{\prime}\right] \operatorname{Vol}_{n} \mathrm{M}_{n} \leqslant \lambda_{1} \ldots \lambda_{n} \operatorname{Vol}_{n} \mathscr{W}(\|\cdot\|) \leqslant 2^{n} \operatorname{Vol}_{n} \mathrm{M}_{n}
$$

Неприятный момент заключается в том, что минимальные векторы не всегда можно достроить до базиса, они порождают более разреженную решетку, что проявляется во множителе $\left[\mathrm{M}_{n}: \mathrm{M}_{n}^{\prime}\right]$.

ЛЕмма 4.2 (Малера [7, гл. 5, лемма 8]). В условиях предложения 4.1 найдется такой базис $\mathbf{e}_{1}, \ldots, \mathbf{e}_{n}$ решетки $\mathrm{M}_{n}$, что

$$
\left\|\mathbf{e}_{1}\right\| \ldots\left\|\mathbf{e}_{n}\right\| \operatorname{Vol}_{n} \mathscr{W}(\|\cdot\|) \leqslant 2^{1-n} n ! \operatorname{Vol}_{n} \mathrm{M}_{n} .
$$

Проясним также роль множителя $\left[\mathrm{M}_{n}: \mathrm{M}_{n}^{\prime}\right]$. Пусть даны некоторая решетка $\mathrm{M}$ и произвольные векторы $\mathbf{v}_{1}, \ldots, \mathbf{v}_{n} \in \mathrm{M}$. Положим

$$
\mathrm{M}_{n}^{\prime}=\left\langle\mathbf{v}_{1}, \ldots, \mathbf{v}_{n}\right\rangle_{\mathbb{Z}}, \quad \mathrm{M}_{n}=\mathrm{M} \cap\left\langle\mathbf{v}_{1}, \ldots, \mathbf{v}_{n}\right\rangle_{\mathbb{R}}
$$

тогда $\mathrm{M}_{n}$ состоит из векторов $\mathbf{v} \in \mathrm{M}$, линейно зависимых над $\mathbb{Z}$ с векторами $\mathbf{v}_{1}, \ldots, \mathbf{v}_{n}$ :

$$
m_{0} \mathbf{v}=m_{1} \mathbf{v}_{1}+\cdots+m_{n} \mathbf{v}_{n}, \quad m_{0}, \ldots, m_{n} \in \mathbb{Z}, \quad m_{0} \neq 0 .
$$

Из теории групп известно, что $\left[\mathrm{M}_{n}: \mathrm{M}_{n}^{\prime}\right] \mathbf{v} \in \mathrm{M}_{n}$, т.е. в приведенной формуле можно положить $m_{0}=\left[\mathrm{M}_{n}: \mathrm{M}_{n}^{\prime}\right]$. Кроме того, пусть $\operatorname{rank} \mathrm{M}_{n}=\operatorname{rank} \mathrm{M}_{n}^{\prime}=\nu>0$; тогда

$$
\left[\mathrm{M}_{n}: \mathrm{M}_{n}^{\prime}\right]=\frac{\mathrm{Vol}_{\nu} \mathrm{M}_{n}^{\prime}}{\mathrm{Vol}_{\nu} \mathrm{M}_{n}} .
$$

Если $\nu=n$, то соотношение между векторами единственно с точностью до целочисленного множителя, и так же можно оценивать все $m_{j}$. Приведем обобщение соответствуюшего утверждения.

ЛЕмма 4.3 [8, теорема 2]. Пусть в евклидовом пространстве $\mathscr{V}$ даны норма $\|\cdot\|$ и векторы $\mathbf{v}_{j}, \quad 0<\left\|\mathbf{v}_{j}\right\| \leqslant A_{j}, \quad 1 \leqslant j \leqslant n, \quad \mathrm{M}=\left\langle\mathbf{v}_{1}, \ldots, \mathbf{v}_{n}\right\rangle_{\mathbb{Z}}-$ решетка ранга $r<n$. Тогда между векторами имеется $n-r$ независимых целочисленных соотношений $\left(m_{i, j} \in \mathbb{Z}\right)$

$$
m_{i, 1} \mathbf{v}_{1}+\cdots+m_{i, n} \mathbf{v}_{n}=0, \quad 1 \leqslant i \leqslant n-r
$$

удовлетворяющих неравенству

$$
\prod_{i=1}^{n-r} \max _{j}\left\{\left|m_{i, j}\right| A_{j}\right\} \leqslant\left(\begin{array}{c}
n \\
r
\end{array}\right)^{\frac{1}{2}} \frac{\mathscr{B}(\|\cdot\|, r)}{\operatorname{Vol}_{r} \mathrm{M}} A_{1} \ldots A_{n}
$$


а если $r=n-1$, то неравенству (считаем $\left.m_{1, j}=m_{j}\right)$

$$
\left|m_{j}\right| A_{j} \leqslant \frac{\mathscr{B}(\|\cdot\|, r)}{\operatorname{Vol}_{r} \mathrm{M}} A_{1} \ldots A_{n}, \quad 1 \leqslant j \leqslant n,
$$

$2 \partial e$

$$
\mathscr{B}(\|\cdot\|, r)=\sup \left\{\operatorname{Vol}_{r}\left\langle\mathbf{e}_{1}, \ldots, \mathbf{e}_{r}\right\rangle_{[0 ; 1]}: \mathbf{e}_{j} \in \mathscr{V},\left\|\mathbf{e}_{j}\right\| \leqslant 1,1 \leqslant j \leqslant r\right\} .
$$

Отметим, что в неравенстве (4.2) множитель $A_{j}$ сокращается, т.е. оценка для $\left|m_{j}\right|$ не зависит от $\left\|\mathbf{v}_{j}\right\|$.

Неравенство (4.2) побуждает рассматривать в пространстве $\mathbb{R}^{n}$ векторов $\left(m_{1}, \ldots, m_{n}\right)$ max-норму с весовыми коэффициентами

$$
|\mathbf{m}|_{A}=\max \left\{\left|m_{j}\right| A_{j}: 1 \leqslant j \leqslant n\right\} ;
$$

тогда (4.2) дает оценку $|\mathbf{m}|_{A}$. Нормы с весовыми коэффициентами будут полезны и в ряде других случаев.

Линейные формы будем записывать как векторы, при этом значение линейной формы а на векторе х обозначим как

$$
\mathbf{a} \cdot \mathbf{x}=a_{1} x_{1}+\cdots+a_{n} x_{n}
$$

Если в векторном пространстве задана норма $\|\cdot\|$, то для пространства линейных форм определена сопряжснная норма $\|\cdot\|^{*}$, для которой выполняется неулучшаемое неравенство

$$
|\mathbf{a} \cdot \mathbf{x}| \leqslant\|\mathbf{a}\|^{*} \cdot\|\mathbf{x}\|
$$

Сопряженной для max-нормы является sum-норма (и наоборот)

$$
\|x\|=\left|x_{1}\right|+\cdots+\left|x_{n}\right|
$$

евклидова норма самосопряжена. Если в таких нормах присутствует набор весовых коэффициентов $\mathscr{A}$, то в сопряженной норме набор весовых коэффициентов $\mathscr{A}^{\prime}$ составлен из обратных величин.

Далее, если явно не оговорено противное, под $|\cdot|$ понимается тах-норма, а под $\|\cdot\|$-sum-норма, и если весовые коэффициенты не указаны, то считаем их равными единице.

Пусть даны линейно независимые над $\mathbb{Z}$ векторы $\mathbf{e}_{1}, \ldots, \mathbf{e}_{n}$, тогда векторы $\mathbf{v}_{i}=$ $m_{i, 1} \mathbf{e}_{1}+\cdots+m_{i, n} \mathbf{e}_{n}, i=1, \ldots, \nu \leqslant n\left(m_{i, j} \in \mathbb{Z}\right)$, можно дополнить до базиса в $\left\langle\mathbf{e}_{1}, \ldots, \mathbf{e}_{n}\right\rangle_{\mathbb{Z}}$, только в том случае, когда набор векторов $\mathbf{m}_{i}=\left(m_{i, 1}, \ldots, m_{i, n}\right) \in$ $\mathbb{Z}^{n}, i=1, \ldots, \nu$, примитивен, т.е. в матрице $\left(m_{i, j}\right)$ все миноры размера $\nu \times \nu$ в совокупности взаимно просты.

Следующая лемма является уточненным вариантом оценки (4.2). 
Лемма 4.4. Пусть даны весовье коэффициенты $A_{1}, \ldots, A_{n}>0, A_{1} \leqslant \ldots$ $\cdots \leqslant A_{n-1}$, линейно независимье векторы $\mathbf{x}_{i} \in \mathbb{Z}^{n}, \quad\left\|\mathbf{x}_{i}\right\|_{A} \leqslant X_{i} \quad(i=$ $1, \ldots, \nu \leqslant n)$, и вектор $\mathbf{b} \in \mathbb{Z}^{n}, \quad b_{n} \neq 0$, линейно зависимый с ними над $\mathbb{Z}$. Тогда имеется нетривиальное соотночение вида

$$
m_{0} \mathbf{b}=m_{1} \mathbf{x}_{1}+\cdots+m_{\nu} \mathbf{x}_{\nu}, \quad m_{0}, \ldots, m_{\nu} \in \mathbb{Z}
$$

удовлетворяющее условиям

$$
\begin{gathered}
\left|m_{0}\right| \leqslant \frac{X_{1} \ldots X_{\nu}}{A_{1} \ldots A_{\nu-1} A_{n}}, \\
\left|m_{j}\right| X_{j} \leqslant \nu|\mathbf{b}|_{A} \cdot \frac{X_{1} \ldots X_{\nu}}{A_{1} \ldots A_{\nu-1} A_{n}}, \quad 1 \leqslant j \leqslant \nu .
\end{gathered}
$$

Если для тах-нормы выполняется $\left|\mathbf{x}_{i}\right|_{A} \leqslant X_{i}, \quad 1 \leqslant i \leqslant r$, то в обоих неравенствах следует поставить справа множитель $\nu^{\nu / 2}$, а во втором при этом убрать множитель $\nu$.

ДокАЗАТЕЛЬСТВо. Поскольку векторы $\mathbf{x}_{i}$ линейно независимы, то коэффициенты $m_{i}$ можно найти следуюшим образом. В матрище координат $\left(x_{i, j}\right)$ выберем ненулевой минор размера $\nu \times \nu$ (пусть в него входят $j \in \mathscr{J}$ ); тогда получаем систему уравнений

$$
m_{1} x_{1, j}+\cdots+m_{\nu} x_{\nu, j}=m_{0} b_{j}, \quad j \in \mathscr{J},
$$

которую можно решить по правилу Крамера и получить

$$
m_{0}=\Delta, \quad m_{i}=\Delta_{i}, \quad i=1, \ldots, \nu,
$$

при этом $\Delta, \Delta_{j} \in \mathbb{Z}, \Delta \neq 0$. Оценим сверху $\left|\Delta_{i}\right|$, для чего умножим столбец $j$ на $A_{j}$, вынесем из $k$-й строки множитель $\nu|\mathbf{b}|_{A}$ при $k=i$ и множитель $X_{k}$ при $k \neq i$. Тогда в строках останутся элементы, сумма модулей которых не превосходит 1 , а такой определитель не превосходит 1. Аналогично оценивается $|\Delta|$. В случае max-нормы следует применить неравенство Адамара.

Для завершения доказательства отметим, что ввиду $b_{n} \neq 0$ среди $n$-х координат векторов $\mathbf{x}_{j}$ есть ненулевые. Поэтому индекс $n$ можно включить в $\mathscr{J}$, откуда следует утверждение леммы.

В заключение параграфа отметим, что для тах-нормы множество $\mathscr{W}(\|\cdot\|)$ является кубом, для sum-нормы - октаэдром с известными формулами для объема, а для евклидовой нормы - обычным шаром,

$$
\operatorname{Vol}_{r} \mathscr{W}\left(\|\cdot\|_{2}\right)=\frac{\pi^{r / 2}}{(r / 2) !}
$$

где $(x) !=\Gamma(x+1)$ обозначает гамма-функцию. 


\section{§5. Высоты}

Общеизвестные факты данного параграфа взяты из [9], [10], а нижние оценки логарифмических высот - из [5], [11].

Пусть $\mathbb{K}^{(\sigma)}$ - вложения поля $\mathbb{K}$ в $\mathbb{C}, 1 \leqslant \sigma \leqslant D$, соответственно имеются $D$ архимедовских нормирований поля $\mathbb{K}$ :

$$
|\alpha|_{\sigma}=\left|\alpha^{(\sigma)}\right|, \quad 1 \leqslant \sigma \leqslant D .
$$

Для комплексно-сопряженных вложений соответствующие нормирования равны, но для удобства будем учитывать их оба. Пронумеруем простые идеалы поля $\mathbb{K}$ индексами $\sigma>D$ и определим неархимедовские нормирования $\mathbb{K}$ по формуле

$$
|\alpha|_{\sigma}=(\operatorname{Norm} \mathfrak{p})^{-k}, \quad k=\operatorname{ord}_{\mathfrak{p}}(\alpha), \quad \mathfrak{p}=\mathfrak{p}_{\sigma}, \quad \sigma>D, \quad \alpha \in \mathbb{K}^{*} .
$$

Тогда имеет место формула произведения

$$
\prod_{\sigma=1}^{\infty}|\alpha|_{\sigma}=1, \quad \alpha \in \mathbb{K}^{*}
$$

Отметим, что $|\alpha|_{\sigma} \neq 1$ только для конечного количества $\sigma$.

Нумерация нормирований удобна при суммировании по всем $\sigma$. При изучении линейной формы от логарифмов мы будем работать с индексами $\sigma=1$ и $\sigma=2$, отвечающими паре комплексно-сопряженных полей. Будем опускать индекс $\sigma=1$, отождествляя $\mathbb{K} \mathrm{c} \mathbb{K}^{(1)}$.

При изучении мультипликативной группы $\mathbb{K}^{*}$ мы всегда будем предполагать $\alpha \neq 0$, не оговаривая этого в дальнейшем особо.

Рассмотрим логарифмическое отображение мультипликативной группы $\mathbb{K}^{*}$ в бесконечномерное пространство $\mathbb{R}^{\infty}$ векторов $\mathbf{x}=\left(x_{\sigma} \in \mathbb{R}: 1 \leqslant \sigma<\infty\right)$, задаваемое формулой

$$
x_{\sigma}=x_{\sigma}(\alpha)=\ln |\alpha|_{\sigma}, \quad 1 \leqslant \sigma<\infty .
$$

Очевидно, это гомоморфизм, а образ $\mathbb{K}^{*}$ лежит в подпространстве $\mathscr{E}=\mathscr{E}(\mathbb{K}) \subseteq$ $\mathbb{R}^{\infty}$, определяемом условиями: $\mathbf{x} \in \mathscr{E}$, если

a) почти все $x_{\sigma}$ равны нулю;

b) $x_{\sigma}=x_{\tau}$ для комплексно-сопряженных $\sigma$ и $\tau$;

c) выполняется аддитивный аналог формулы произведения: $\sum_{\sigma=1}^{\infty} x_{\sigma}=0$.

Будем называть $\mathscr{E}$ логарифмическим пространством.

ЗАмЕчАниЕ. Обычно комплексно-сопряженныепары $x_{\sigma}=x_{\tau}$ заменяют одним числом $2 x_{\sigma}$, но нам кажется удобнее учитывать все $\sigma$. Далее, пространство $\mathscr{E}$ бесконечномерно, но это только формальная конструкция. Реально всегда приходится работать только в некотором конечномерном координатном подпространстве, конкретно в данной работе можно обойтись только всеми архимедовскими координатами и теми $p$-адическими координатами, где хотя бы для одного из чисел $\alpha_{j}$ имеем $\left|\alpha_{j}\right|_{\sigma} \neq 1$.

Отметим, что для логарифмических образов $\mathbf{x}(\alpha)$ выполняется

$$
x_{\sigma} \in \ln \left(\operatorname{Norm} \mathfrak{p}_{\sigma}\right) \mathbb{Z}, \quad \sigma>D .
$$


Абсолютную логарифмическую высоту числа $\alpha \in \mathbb{K}$ зададим формулой

$$
\mathrm{h}(\alpha)=\frac{1}{D} \sum_{\sigma=1}^{\infty} \max \left\{0, \ln |\alpha|_{\sigma}\right\}=\left.\frac{1}{2 D} \sum_{\sigma=1}^{\infty}|\ln | \alpha\right|_{\sigma} \mid
$$

(последнее равенство верно вследствие формулы произведения). Она задает норму в логарифмическом пространстве. С точностью до множителя это - sum-норма в $\mathscr{E}$, т.е. $\mathrm{h}(\alpha)=(2 D)^{-1}\|\mathbf{x}(\alpha)\|$. Абсолютной высота названа потому, что она не зависит от поля, в котором можно разместить число $\alpha$. Для $\alpha \in \mathbb{Z}, \alpha \neq 0$, имеем $\mathrm{h}(\alpha)=\ln |\alpha|$.

По теореме Кронекера ядром логарифмического отображения является группа корней из 1 поля $\mathbb{K}$; эта группа конечна, циклична; образ $\mathbb{K}^{*}$ является решеткой B $\mathscr{E}$.

Теперь построим конструкцию, которую назовем расширенным логарифмическим пространством. Она была детально и с большей степенью общности описана в [5]. Для этого в прежнем пространстве $\mathbb{R}^{\infty}$, содержащем $\mathscr{E}$, заменим $\mathbb{R}$-компоненты, соответствуюшие координатам $\sigma=1, \varkappa$, на $\mathbb{C}$-компоненты. Соответствуюшие координаты будут $z_{\sigma}=x_{\sigma}+i y_{\sigma}$. Для координат $x_{\sigma}$ сохраняются прежние условия (a)-(c), а для $y_{\sigma}$ должно быть:

d) $y_{\sigma}=-y_{\tau}$, если $\sigma$ и $\tau$ комплексно-сопряжены.

Полученное пространство назовем расширенным логарифмическим пространством $\mathscr{E}_{\varkappa}=\mathscr{E}_{\varkappa}(\mathbb{K})$. Можно считать, что $\mathscr{E} \subseteq \mathscr{E}_{\varkappa}$, относя к $\mathscr{E}$ векторы, имеющие все $y_{\sigma}=0$. Имеется естественное отображение $\mathscr{E}_{\varkappa}$ В $\mathscr{E}$ - проекция, аннулирующая все $y_{\sigma}$.

Теперь опишем, как связана группа $\mathbb{K}^{*}$ с пространством $\mathscr{E}_{\varkappa}$. Для этого координаты $x_{\sigma}$ зададим прежним образом, а координаты $y_{\sigma}$ определим как $y_{\sigma}=\operatorname{Arg} \alpha^{(\sigma)}$. Другими словами, $z_{\sigma}=\operatorname{Ln} \alpha^{(\sigma)}, \sigma=1, \varkappa$. При этом, строя соответствуюшую решетку в $\mathscr{E}_{\varkappa}$, будем брать все возможные значения логарифма. Полученное множество точек в $\mathscr{E}_{\varkappa}$ обозначим через $\mathrm{M}_{\varkappa}(\mathbb{K})$. Тогда для $\mathbf{x} \in \mathrm{M}_{\varkappa}(\mathbb{K})$ с некоторым $\alpha \in \mathbb{K}^{*}$ выполняется

$$
\begin{cases}\exp \left(x_{\sigma}\right)=|\alpha|_{\sigma}, & \sigma>\varkappa, \\ \exp \left(z_{\sigma}\right)=\alpha^{(\sigma)}, & \sigma \leqslant \varkappa,\end{cases}
$$

что позволяет восстанавливать $\alpha$ по его образу. Таким образом, теперь само расширенное логарифмическое отображение неоднозначно, но существует однозначное обратное отображение $\mathrm{M}_{\varkappa}(\mathbb{K})$ на $\mathbb{K}^{*}$, определяемое по указанным формулам.

Как отмечалось в $\S 3$, если $\mathbb{K} \subseteq \mathbb{R}$, то для доказательства теорем достаточно ограничиться рассмотрением только положительных чисел $\alpha_{j}$ с действительными значениями логарифмов. Будем обозначать соответствующие объекты как $\mathbb{K}^{+}$, $\mathscr{E}_{\varkappa}\left(\mathbb{K}^{+}\right), \mathrm{M}_{\varkappa}\left(\mathbb{K}^{+}\right)$. В то же время вспомогательные леммы будут доказываться и для общего случая, для которого утверждения могут отличаться от случая $\mathbb{K}^{+}$.

Для изучения множества $\mathrm{M}_{\varkappa}(\mathbb{K})$ в [5] были рассмотрены несколько норм в пространстве $\mathscr{E}_{\varkappa}$, одна из которых имела следующий вид. Для $\vartheta>0$ положим

$$
\mathrm{H}_{\vartheta}^{*}(\mathbf{x})=\max \left\{\max _{\sigma \leqslant \varkappa}\left|z_{\sigma}\right|, \frac{\vartheta}{2 \varkappa} \sum_{\sigma>\varkappa}\left|x_{\sigma}\right|\right\}
$$


тогда $\mathrm{H}_{\vartheta}^{*}(\alpha)=\mathrm{H}_{\vartheta}^{*}(\mathbf{x})$, если $\mathbf{x}=\mathbf{x}(\alpha) \in \mathscr{E}_{\varkappa}$ является некоторым логарифмическим образом числа $\alpha$. Упростим это выражение, суммируя по всем $\sigma \geqslant 1$. Получим

$$
\mathrm{H}_{\vartheta}(\alpha)=\mathrm{H}_{\vartheta}(\mathbf{x})=\max \left\{\frac{D \vartheta}{\varkappa} \mathrm{h}(\alpha), \max _{\sigma \leqslant \varkappa}\left|\ln \alpha^{(\sigma)}\right|\right\} .
$$

Назовем $\mathrm{H}_{\vartheta}(\alpha)$ расширенной логарифмической выссотой $\alpha$ (с весовым коэффициентом $\vartheta)$. Отметим, что в случае $\mathbb{K}^{+}$мы имеем просто $\mathrm{H}_{\varkappa}(\alpha)=D \mathrm{~h}(\alpha)$.

В [5] рассматривался обший случай, когда $\varkappa$ принимает значения $1 \leqslant \varkappa \leqslant D$, а $\vartheta$ меняется в диапазоне от нуля до бесконечности. В данной работе для простоты изложения мы рассматриваем только $\varkappa \in\{1,2\}$, поэтому $\max \left\{\left|\ln \alpha^{(\sigma)}\right|: \sigma \leqslant \varkappa\right\}=$ $|\ln \alpha|$.

В ряде предшествуюших работ рассматривался параметр

$$
\frac{1}{E}=\max _{j}\left\{\frac{1}{\mathrm{H}_{\varkappa}\left(\alpha_{j}\right)} \max _{\sigma \leqslant \varkappa}\left|\ln \alpha_{j}^{(\sigma)}\right|\right\},
$$

по порядку соответствуюший $\vartheta$. Чем больше $E$, тем лучше получаются оценки линейных форм. Однако зависимость нормы $\mathrm{H}_{\vartheta}(\alpha)$ от $\vartheta$ оказалась полезной, поскольку для этой нормы удалось доказать нетривиальную оценку для последовательных минимумов решетки $\mathrm{M}_{\varkappa}(\mathbb{K})$, причем в виде, необходимом для оценок линейных форм от логарифмов.

ПРЕДЛОЖЕНИЕ 5.1 [5, теорема 6.2]. Пусть $\mathbf{v}_{1}, \ldots, \mathbf{v}_{n}$-линейно независимые векторы из $\mathrm{M}_{\varkappa}(\mathbb{K})$. Положим

$$
\mathrm{M}_{n}=\mathrm{M}_{\varkappa}(\mathbb{K}) \cap\left\langle\mathbf{v}_{1}, \ldots, \mathbf{v}_{n}\right\rangle_{\mathbb{R}}, \quad \mathrm{M}_{n}^{\prime}=\left\langle\mathbf{v}_{1}, \ldots, \mathbf{v}_{n}\right\rangle_{\mathbb{Z}}, \quad N=\left[\mathrm{M}_{n}: \mathrm{M}_{n}^{\prime}\right] .
$$

Тогда

$$
\begin{gathered}
\mathrm{H}_{\vartheta}^{*}\left(\mathbf{v}_{1}\right) \ldots \mathrm{H}_{\vartheta}^{*}\left(\mathbf{v}_{n}\right) \xi_{0}^{*} \max \left\{6, n-1, \frac{D}{\varkappa} \ln \left(4.64 \frac{D}{\varkappa}\right)\right\}>N, \\
\xi_{0}^{*}=\left(2+\frac{1}{2 \vartheta}\right)^{n}\left(e^{n /(1+4 \vartheta)} n\right)^{\varkappa} .
\end{gathered}
$$

Для согласования с обозначениями $(2.1)$ считаем в дальнейшем $\vartheta=\varkappa и \mathrm{H}_{\vartheta}(\alpha)=$ $\mathrm{H}_{\varkappa}(\alpha)$. Упростим формулу для $\xi_{0}^{*}$ с учетом $\varkappa \leqslant 2$ и запишем оценку предложения 5.1 в виде, достаточном для наших приложений (случай $n=1$ см. далее):

$$
\begin{gathered}
A_{1} \ldots A_{n} \xi_{0}(n) \frac{D}{N} \ln \left(e^{4+n} D\right)>1, \\
\xi_{0}(0)=1, \quad \xi_{0}(1)=1.5, \quad \xi_{0}(\nu)=3.1^{\nu} \nu^{\varkappa} / \varkappa, \quad \nu \geqslant 2
\end{gathered}
$$

(для линейно независимых над $\mathbb{Z}$ чисел $\ln \alpha_{1}, \ldots, \ln \alpha_{n}$ ). Неравенство (5.1) можно использовать и для оценки $A_{1} \ldots A_{\nu}(1 \leqslant \nu \leqslant n)$, если заменить $N$ на 1 и $n$ на $\nu$ :

$$
A_{1} \ldots A_{\nu} \xi_{0}(\nu) D \ln \left(e^{4+\nu} D\right)>1 .
$$

Отметим также, что в $(5.1),(5.2)$ величину $\ln \left(e^{4+n} D\right)$ можно заменить на $C_{0} / 2$ или $W_{0} / 2$ из (2.2) (по поводу $W_{0}$ см. (6.3)).

Случай $n=1$ был рассмотрен ранее в [11], где была получена несколько более точная оценка [11, следствие 4]. 
ПРЕДЛОЖЕНИЕ 5.2. При $\alpha \in \mathbb{K}^{*}, \operatorname{deg} \alpha=d>1$, выполняется

$$
\max \{d \mathrm{~h}(\alpha),|\ln \alpha|\} \geqslant \frac{8}{9 d \ln (2.2 d)} .
$$

Перепишем неравенство из предложения 5.2 в виде

$$
\frac{1}{A_{j}} \leqslant 1.5 D \ln (e D)
$$

верном и при $D=1$, так как здесь либо $\mathrm{h}(\alpha) \geqslant \ln 2$, либо $\alpha= \pm 1$. Этим обусловлено значение $\xi_{0}(1)=1.5$.

В 1933 г. Лемером было найдено число $\alpha=1.176 \ldots$ степени $d=10$, являющееся корнем уравнения

$$
x^{10}+x^{9}-x^{7}-x^{6}-x^{5}-x^{4}-x^{3}+x+1=0,
$$

и имеющее наименьшую известную логарифмическую высоту $d \mathrm{~h}(\alpha)=0.162 \ldots$ среди алгебраических чисел, отличных от корней из 1. Этим обусловлен выбор константы 0.16 в условии (2.4).

В лемме 4.2 приведено утверждение для решеток и норм общего вида, где оставлены параметры $\mathscr{B}(\|\cdot\|, r), \mathrm{Vol}_{r} \mathrm{M}$. При этом важно лишь их отношение, и, кроме того, мы можем сами выбирать евклидову норму, определяющую объемы. Укажем оценки $\mathscr{B}(\|\cdot\|, r), \mathrm{Vol}_{r} \mathrm{M}$ для решеток в $\mathscr{E}_{\varkappa}(\mathbb{K})$, используюшиеся в дальнейшем. При этом в качестве евклидовой нормы возьмем

$$
\|\mathbf{v}\|_{2}^{2}=\sum^{(*)} y_{\sigma}^{2}+\sum_{\sigma=1}^{\infty} x_{\sigma}^{2},
$$

где в сумме $\sum^{(*)}$ из пары комплексно-сопряженных координат берем только одну (для $\mathbb{K}^{+}$эта составляюшая отсутствует).

Лемма 5.3. В $\mathscr{E}_{\varkappa}(\mathbb{K})$ для нормы $\|\cdot\|=\mathrm{H}_{\varkappa}(\cdot)$ и скалярного произведения, индуцированного нормой (5.4) $c k$ слагаемьмм $y_{\sigma}^{2}$ в $\sum^{(*)}$, имеет место оцен$\kappa a$

$$
\mathscr{B}(\|\cdot\|, r) \leqslant(k+2)^{r / 2} \text {. }
$$

Для любой $r$-мерной решетки $\mathrm{M} \subseteq \mathrm{M}_{\varkappa}(\mathbb{K}), \quad \mathbb{K} \neq \mathbb{Q}$, выполняется

$$
\operatorname{Vol}_{r} \mathrm{M} \geqslant \frac{\pi^{r / 2}}{(r / 2) !}\left(\frac{9}{8} D^{3 / 2} \ln (e D)\right)^{-r}
$$

В случае $\mathbb{K}=\mathbb{Q}$ выполняется

$$
\mathrm{Vol}_{r} \mathrm{M} \geqslant P_{1} \ldots P_{r}
$$

$P_{1}=\ln 2, \quad P_{2}=\ln 3, \ldots, P_{9}=\ln 23, P_{10}=\pi, P_{11}=\ln 29, \ldots$ (перебираются логарифмы простых чисел), а в случае $\mathbb{Q}^{+}$следует удалить из последовательности число $\pi$. 
В случае $\mathbb{K} \neq \mathbb{Q}, \quad k \leqslant 1$, а также в случае $\mathbb{Q}^{+}, \quad k=0$, выполняется неравенство

$$
\frac{\mathscr{B}(\|\cdot\|, r)}{\operatorname{Vol}_{r} \mathrm{M}} \leqslant 2.2\left(\frac{r}{2}\right) !\left(\frac{3}{\pi}\right)^{r / 2}\left(\frac{9}{8} D^{3 / 2} \ln (e D)\right)^{r} .
$$

Отметим, что (5.7) отличается от (5.5) множителем 2.2 , который добавлен только для того, чтобы при $D=1$ правая часть (5.6) была меньше правой части (5.5) и в случае $r<4$.

Доказательство приводится в $\S 22$.

Теперь напомним понятие высоты в векторных $\mathbb{K}$-пространствах. Локальные и абсолютно логарифмические высоты набора чисел $\left\{\beta_{j} \in \mathbb{K}: j \in \mathscr{J}\right\}$ определяются следуюшим образом:

$$
\begin{gathered}
\mathrm{h}^{(\sigma)}\left(\left\{\beta_{j}\right\}\right)=\ln \max \left\{\left|\beta_{j}\right|_{\sigma}: j \in \mathscr{J}\right\}, \quad \sigma \geqslant 1, \\
\mathrm{~h}\left(\left\{\beta_{j}\right\}\right)=\frac{1}{D} \sum_{\sigma=1}^{\infty} \mathrm{h}^{(\sigma)}\left(\left\{\beta_{j}\right\}\right) .
\end{gathered}
$$

Высота $\mathrm{h}(\cdot)$ проективна, т.е. для $\lambda \in \mathbb{K}^{*}$ имеем $\mathrm{h}\left(\left\{\lambda \beta_{j}\right\}\right)=\mathrm{h}\left(\left\{\beta_{j}\right\}\right)$, что следует из формулы произведения.

В дальнейшем нам придется рассматривать значения многочленов от многих переменных в точке $\bar{\alpha}$. Здесь набор $\left\{\beta_{j}\right\}$ будет сформирован из чисел вида $\bar{\alpha}^{\bar{l}}$, где $\bar{l}$ принадлежит некоторому множеству мультистепеней $\mathscr{L} \subseteq \mathscr{W} \subseteq \mathbb{R}^{n}$. Для удобства оценок в этом случае введем следующие обозначения. Для заданного вектора $\bar{\alpha} \in\left(\mathbb{K}^{*}\right)^{n}$ и множества $\mathscr{W} \subseteq \mathbb{R}^{n}$ положим

$$
\begin{gathered}
\mathrm{h}^{(\sigma)}(\bar{\alpha}, \mathscr{W})=\sup \left\{\bar{w} \cdot \ln |\bar{\alpha}|_{\sigma}: \bar{w} \in \mathscr{W}\right\}, \quad \sigma \geqslant 1, \\
\mathrm{~h}(\bar{\alpha}, \mathscr{W})=\frac{1}{D} \sum_{\sigma=1}^{\infty} \mathrm{h}^{(\sigma)}(\bar{\alpha}, \mathscr{W}) .
\end{gathered}
$$

Лемма $5.4[1$, лемма 3.2$]$. Выссота $\mathrm{h}(\bar{\alpha}, \mathscr{W})$ обладает следующими свойствами:

(i) если $\mathscr{V} \subseteq \mathscr{W}, \operatorname{mo~} \mathrm{h}(\bar{\alpha}, \mathscr{V}) \leqslant \mathrm{h}(\bar{\alpha}, \mathscr{W})$;

(ii) $\mathrm{h}\left(\bar{\alpha}, r^{\mathscr{W}}\right)=r \mathrm{~h}(\bar{\alpha}, \mathscr{W}), r>0$;

(iii) $\mathrm{h}(\bar{\alpha}, \mathscr{V}+\mathscr{W})=\mathrm{h}(\bar{\alpha}, \mathscr{V})+\mathrm{h}(\bar{\alpha}, \mathscr{W})$;

(iv) $\mathrm{h}(\bar{\alpha},\{\bar{v}\})=0, \quad \bar{v} \in \mathbb{R}^{n}$;

(v) $\mathrm{h}(\bar{\alpha}, \mathscr{W}+\bar{v})=\mathrm{h}(\bar{\alpha}, \mathscr{W}), \quad \bar{v} \in \mathbb{R}^{n}$;

(vi) $\mathrm{h}(\bar{\alpha}, \mathscr{W})$ не зависит от выбора поля, содержащего числа $\alpha_{j}$.

\section{§6. Неравенство Лиувилля}

В данном параграфе будет доказано, что теорема 2.1 верна при $n=1$, а также при сравнительно небольших значениях $B$.

В теории диофантовых приближений неравенства, непосредственно получающиеся из формулы произведения, обычно называют лиувиллевьми. Оказывается, параметр $B$ вида (1.3) очень удобен для записи подобного рода оценок. 
Лемма 6.1 [1, лемма 13.1]. Пусть в обозначениях теоремы $2.1 \Lambda \notin 2 \pi i \mathbb{Z}$. Тогда

$$
\left|e^{\Lambda}-1\right| \geqslant 2^{1-D} e^{-n B A_{n}} .
$$

Ввиду отсутствия формальных нижних границ для учитьваемых параметров в оценке $\Lambda$ некоторые прежде очевидные утверждения требуют проверки.

ЛЕмма 6.2. Теорема 2.1 верна при $|\Lambda| \geqslant 0.001$.

ДокАЗАТЕЛЬСТво. Из (5.2) имеем $\xi_{0}(n) D \Omega C_{0} \geqslant 1$, поэтому в правой части (2.3) остается еше достаточно большой множитель, что доказывает утверждение леммы.

Таким образом, далее можно считать $|\Lambda|<0.001$. Лемма 6.1 в этом случае приводит к неравенству

$$
|\Lambda| \geqslant \frac{1}{2}\left|e^{\Lambda}-1\right| \geqslant \exp \left(-D \ln 2-n B A_{n}\right)
$$

Теперь рассмотрим случай, когда лиувиллева оценка хуже, чем (2.3). Тогда

$$
-D \ln 2-n B A_{n} \leqslant-C(n) D^{2} \Omega C_{0} W_{0},
$$

или с учетом (5.2)

$$
n B A_{n} \geqslant C(n) D^{2} \Omega C_{0} W_{0}-D \ln 2 \geqslant 0.99 C(n) D^{2} \Omega C_{0} W_{0} .
$$

После сокращения на $n A_{n}$ получаем

$$
B \geqslant 0.99 \frac{1}{n} C(n) D^{2} A_{1} \ldots A_{n-1} C_{0} W_{0} .
$$

При этом показательная часть $C(n)$ имеет вид $\left(4 e^{2}\right)^{n}$. В частности, применяя оценку (5.2) для множителя $D A_{1} \ldots A_{n-1} C_{0} / 2$, получаем $B \geqslant e^{2 n+8} D$. Это позволяет использовать неравенства $(5.1),(5.2)$ с величиной $W_{0} / 2$ вместо $\ln \left(e^{4+\nu} D\right)$. Поэтому из (6.1) следует

$$
B \geqslant 1.98 C(n) C_{0} D / n \xi_{0}(n-1),
$$

а для $W_{0}$ имеем неравенство

$$
W_{0} \geqslant 2.25 n+1.5 \ln n+13+2 \ln D
$$

непосредственно проверяемое при малых $n$ и получающееся асимптотически для больших $n$, поскольку для основания показательной зависимости от $n$ в (6.2) имеем $\ln \left(4 e^{2} / 3.1\right)>2.25$.

Кроме того, из (6.1) следует также оценка

$$
A_{1} \ldots A_{n-1} \leqslant B\left(0.99 \frac{1}{n} C(n) D^{2} C_{0} W_{0}\right)^{-1} .
$$

Таким образом, в дальнейшем можно считать неравенство (6.1) выполненным (иначе теорема 2.1 верна в силу лиувиллевой оценки), значит, выполнены также неравенства (6.2)-(6.4). 
Лемма 6.3. Теорема 2.1 верна при $n=1$.

ДокАЗАТЕЛЬСТвО. При $n=1$ имеем

$$
|\Lambda|=\left|b_{n} \ln \alpha_{n}\right| \geqslant\left|\ln \alpha_{n}\right|
$$

так что здесь можно считать $B=1$, и поэтому теорема 2.1 следует из лиувиллевой оценки.

В заключение параграфа отметим, что в приведенных вычислениях, а также в следующих далее оценках степенная зависимость от $D$ оптимальна. Впервые подобная зависимость была установлена для произвольного $n$ в [2].

\section{§7. Индуктивное предположение}

Вся статья, кроме $\S 21,22$, будет посвящена доказательству теоремы 2.1. Мы будем доказывать ее индукцией по $n$ - числу логарифмов в линейной форме $\Lambda$, предполагая ее доказанной для меньшего числа логарифмов. Основание индукции $(n=1)$ было доказано в $\S 6$, так что далее считаем $n \geqslant 2$.

Ясно также, что мы можем считать набор чисел $b_{j}$ примитивным, иначе, сократив их на общий множитель, мы уменьшим $B$ и улучшим оценку линейной формы.

Объясним еще одно допущение, вводимое в данном параграфе. Пользуясь свойствами логарифмической функции, можно уменьшить количество логарифмов в линейной форме $\Lambda$ :

$$
\Lambda=b_{1} \ln \alpha_{1}+\cdots+b_{n} \ln \alpha_{n}=\left(m_{1} \ln \vartheta_{1}+\cdots+m_{r} \ln \vartheta_{r}\right) / m_{0},
$$

например записать $\Lambda=\ln \vartheta, \vartheta=\bar{\alpha}^{\bar{b}}$. Обычно это сопровождается увеличением высот чисел $\vartheta_{i}$ и только ухудшает оценку. Однако если увеличение высот невелико, то теорему можно непосредственно доказать из индуктивного предположения для $r<n$. Опишем эту ситуацию, принимая за основу рассуждения из [4].

Напомним, что в теореме 2.1 числа $\ln \alpha_{j}$ предполагаются линейно независимыми над $\mathbb{Z}$. Пусть теперь при некотором $r(1 \leqslant r<n)$ существует набор векторов

$$
\bar{Z}_{i}=\left(z_{i, 1}, \ldots, z_{i, n}\right) \in \mathbb{Z}^{n}, \quad 1 \leqslant i \leqslant r,
$$

удовлетворяющих условиям:

(i) $\bar{Z}_{1}, \ldots, \bar{Z}_{r}$ линейно независимы над $\mathbb{Z}$;

(ii) с некоторым примитивным набором $m_{0}, \ldots, m_{r} \in \mathbb{Z}, m_{0} \neq 0$, выполняется

$$
m_{0} \bar{b}=m_{1} \bar{Z}_{1}+\cdots+m_{r} \bar{Z}_{r}
$$

(iii) для sum-нормы с весовыми коэффициентами $A_{j}$ из условий теоремы 2.1 выполняется

$$
\left\|\bar{Z}_{1}\right\|_{A} \ldots\left\|\bar{Z}_{r}\right\|_{A} \leqslant \frac{1}{2}\left(4 e^{2}\right)^{n-r} A_{1} \ldots A_{n} .
$$

ЛЕмма 7.1. При выполнении условий (i)-(iii) справедливость теоремы 2.1 для $n$ следует из ее справедливости для $r<n$. 
ДокАЗАТЕЛЬСТво. Определим алгебраические числа $\vartheta_{i}$ и их логарифмы по формулам

$$
\ln \vartheta_{i}=\bar{Z}_{i} \cdot \ln \bar{\alpha}=z_{i, 1} \ln \alpha_{1}+\cdots+z_{i, n} \ln \alpha_{n}, \quad 1 \leqslant i \leqslant r
$$

Тогда, очевидно, равенство (7.1) выполнено с коэффициентами $m_{i}$ из (7.2), и при этом логарифмы $\ln \vartheta_{i}$ линейно независимы над $\mathbb{Z}$.

Из определения чисел $A_{j}$ и свойств высоты имеем

$$
\mathrm{H}_{\varkappa}\left(\vartheta_{i}\right) \leqslant A_{i}^{\prime}=\left\|\bar{Z}_{i}\right\|_{A}, \quad 1 \leqslant i \leqslant r .
$$

Поскольку $b_{n} \neq 0$, среди чисел $z_{i, n}, 1 \leqslant i \leqslant r$, есть ненулевое с соответствуюшим $m_{i} \neq 0$. Без ограничения обшности можно считать, что $z_{r, n} \neq 0, m_{r} \neq 0$. Тогда $A_{r}^{\prime} \geqslant\left|z_{r, n}\right| A_{n} \geqslant A_{n}$.

Положим теперь по аналогии с обозначениями $\S 2$

$$
B^{\prime}=\max \left\{\left|m_{i}\right| A_{i}^{\prime} / A_{r}^{\prime}: 1 \leqslant i \leqslant r\right\}, \quad \Omega^{\prime}=A_{1}^{\prime} \ldots A_{r}^{\prime},
$$

и оценим с помошью леммы 4.3 и неравенства (7.3) число $B^{\prime}$. Имеем

$$
B^{\prime} A_{r}^{\prime}=\max \left\{\left|m_{i}\right| A_{i}^{\prime}: 1 \leqslant i \leqslant r\right\} \leqslant \frac{r B \Omega^{\prime}}{A_{1} \ldots A_{r-1}} \leqslant \frac{1}{2} r B\left(4 e^{2}\right)^{n-r} \frac{A_{1} \ldots A_{n}}{A_{1} \ldots A_{r-1}} .
$$

Сокращая на $A_{r}^{\prime} \geqslant A_{n}$ и применяя к $1 /\left(A_{1} \ldots A_{r-1}\right)$ неравенство $(5.2)$, получаем с учетом (6.4), что

$$
B^{\prime} \leqslant \frac{1}{2} r B\left(4 e^{2}\right)^{n-r} \xi_{0}(r-1) D \ln \left(e^{n+4} D\right) \frac{2 n B}{C(n) D^{2} C_{0} W_{0}} \leqslant B^{2} .
$$

Аналогично, с помошью леммы 4.3 и неравенств (7.3), (5.2), (6.4) оценивается $m_{0}$ :

$$
\begin{aligned}
\left|m_{0}\right| & \leqslant\left\|\bar{Z}_{1}\right\|_{A} \ldots\left\|\bar{Z}_{r}\right\|_{A} \frac{1}{A_{1} \ldots A_{r-1} A_{n}} \\
& \leqslant \frac{1}{2}\left(4 e^{2}\right)^{n-r} A_{1} \ldots A_{n-1} \xi_{0}(r-1) D \ln \left(e^{n+4} D\right) \\
& \leqslant \frac{1}{2}\left(4 e^{2}\right)^{n-r} \frac{2 n B}{C(n) D^{2} C_{0} W_{0}} \xi_{0}(r-1) D \ln \left(e^{n+4} D\right) \leqslant B .
\end{aligned}
$$

Применим теперь теорему 2.1 к форме $m_{0} \Lambda$ из (7.1) с числами $\vartheta_{i}$ и $m_{i}$ и получим (опять с учетом (7.3))

$$
\begin{aligned}
-\ln |\Lambda| & \leqslant \ln \left|m_{0}\right|+C(r) D^{2} \Omega^{\prime} C_{0} \ln \left(1.5 e B^{\prime} D \ln (e D)\right) \\
& \leqslant \ln B+C(r) \frac{1}{2}\left(4 e^{2}\right)^{n-r} D^{2} \Omega C_{0} \ln \left(1.5 e B^{2} D \ln (e D)\right) \\
& \leqslant W_{0}+4 e^{2} C(n-1) D^{2} \Omega C_{0} W_{0} \leqslant C(n) D^{2} \Omega C_{0} W_{0},
\end{aligned}
$$


что доказьвает теорему 2.1 в рассматриваемом случае при заданном $n$. Здесь мы использовали легко проверяемое неравенство

$$
\frac{C(n)}{C(n-1)}>4 e\left(1+\frac{1}{n}\right)^{n+2}>4 e^{2}\left(1+\frac{1}{n}\right)
$$

Итак, в дальнейшем считаем, что условия леммы 7.1 не выполнены, т.е. не сушествуют $r(1 \leqslant r \leqslant n)$ и векторы $\bar{Z}_{i}$ с условиями (i)-(iіi). Этим наша индуктивная схема несколько отличается от схемы Бейкера-Вюстхольца, которые максимально редуцировали линейную форму $\Lambda$ и далее работали с укороченной формой. Предлагаемая нами схема экономит обозначения.

Отметим, что это одно из мест в доказательстве, где за счет применения весовых коэффициентов удалось сохранить зависимость оценки $\Lambda$ от числа $B$ из (1.3), не переходя к $B^{*}$ из (1.4).

Индуктивное предположение понадобится нам только в конще доказательства в $\oint 20$.

Отметим также, что идея редукции была предложена в статье [12] в 1949 г. для перехода от $n=3$ к $r=2$. Конечно, если линейная форма $\Lambda$ допускает понижение числа переменных, то это имеет смысл сделать. Главная трудность состоит в том, как использовать информацию о том, что форма не упрощается. Недавно выяснилось, что это условие является ключевым в теореме об оценках кратностей нулей (см. $§ 19)$.

\section{§8. Обобщенные биномиальные многочлены}

Данный параграф̆ является вспомогательным. В нем приводятся сведения о базисных многочленах, обладающих нужными арифметическими свойствами. Положим

$$
\Delta(z, 0)=1, \quad \Delta(z, k)=\frac{1}{k !} z(z+1) \ldots(z+k-1), \quad k=1,2, \ldots,
$$

и для фиксированного $H \in \mathbb{N}$ определим

$$
\Delta(z, l, H)=(\Delta(z, H))^{\lambda} \Delta(z, h), \quad l=\lambda H+h, \quad 0 \leqslant h \leqslant H, \quad l \in \mathbb{N}_{0} .
$$

Будем называть $\Delta(z, l, H)$ обобщенными биномиальными многочленами.

Отметим, что для степеней $l>0$, деляшихся на $H$, возможно двоякое представление $l=\lambda H+H=(\lambda+1) H$, однако легко видеть, что $\Delta(z, l, H)$ не зависит от способа представления $l$. В оценках вьгоднее брать первую запись с меньшим значением $\lambda$. Очевидно, $\operatorname{deg} \Delta(z, l, H)=l$. Эти многочлены являются модификацией аналогичных многочленов, ранее введенных Фельдманом и Бейкером. Под $\Delta^{(m)}(c z, l, H)$ будем понимать производную $\Delta(c z, l, H)$ по параметру $z$.

В [13] доказано следующее свойство многочленов $\Delta(z, l, H)$.

Лемма 8.1. Пусть $H, L_{0} \in \mathbb{N} ; \quad m_{0}, L^{\prime} \in \mathbb{N}_{0} ; \quad L_{0}=L^{\prime} H+H_{1}, \quad 0 \leqslant H_{1} \leqslant H ;$ $d_{0}\left(m_{0}\right)$ - наименьший общий знаменатель чисел

$$
\frac{1}{m !} \Delta^{(m)}(x, l, H), \quad x \in \mathbb{Z}, \quad 0 \leqslant l \leqslant L_{0}, \quad 0 \leqslant m \leqslant m_{0} .
$$


Тогда

$$
d_{0}\left(m_{0}\right) \leqslant H !^{L^{\prime}} H_{1} !\left(2.8361 e \frac{m_{0}+L^{\prime}+1}{L_{0}}\right)^{L_{0}}
$$

a maxжe

$$
d_{0}\left(m_{0}\right) \leqslant \exp \left(1.03883 H m_{0}\right) .
$$

Отметим, что оценка (8.1) фельдмановского типа, степенная по $m_{0}$, несколько точнее, чем оценка бейкеровского типа (8.2). Для простоты мы будем использовать оценку (8.2), лучше приспособленную к оценкам остальных компонент.

В дальнейшем мы будем брать значения $x \in \frac{1}{K} \mathbb{Z}, K=2^{t}$. Для избежания дробных значений многочленов мы будем заранее брать $\Delta(K x, l, H)$. Это полезно тем, что здесь не надо еще домножать $\Delta^{(m)}(x, l, H)$ на

$$
\Pi(K, l)=\prod_{p \mid K} p^{[l /(p-1)]}
$$

( $p$ - простое число). Отметим, что при $K=2^{t}$ выполнено П $(K, l)=2^{l}$.

Кроме леммы 8.1 в [13] была доказана оценка модуля $\left|\Delta^{(m)}(z, l, H)\right|$. Здесь мы получим усиление оценки модуля.

Лемма 8.2. Пусть $H \in \mathbb{N}, \quad z, c \in \mathbb{C}, c \neq 0$. Тогдa

$$
\begin{aligned}
\left|\frac{1}{\tau ! m !} c^{-m} \Delta^{(m+\tau)}(c z, l, H)\right| & =\delta(l, m, \tau)\left(e\left(1+|c| \frac{|z|+1}{H}\right)\right)^{l}, \\
\sum_{l=0}^{\infty} \sum_{m+\tau \leqslant l} \delta(l, m, \tau) & \leqslant \frac{e}{e-1} e^{H / e}
\end{aligned}
$$

величины $\delta(\cdot)$ также зависят от $c, z, H$.

ДоКАЗАТЕЛЬСТВО. Полиномиальная составляющая в левой части первой строки (8.3) не превосходит $(|c z|+H-1)^{l^{\prime}}|c|^{\tau}$, где $l^{\prime}=l-m-\tau$. Возникающие при дифференцировании факториалы и количество слагаемых оцениваются сверху как $\frac{(m+\tau) !}{m ! \tau !}\left(\begin{array}{c}l \\ m+\tau\end{array}\right)$. Суммирование по $m+\tau \leqslant l$ (т.е. $m+\tau+l^{\prime}=l$ ) дает

$$
\sum_{m+\tau+l^{\prime}=l} \frac{l !}{m ! \tau ! l^{\prime} !}(|c z|+H-1)^{l^{\prime}}|c|^{\tau} 1^{m}=((|c z|+H-1)+|c|+1)^{l} .
$$

После вынесения за скобки множителя $(H / e)^{l}$ получаем первую строку (8.3).

Суммирование по $l=H \lambda+h \geqslant 0$ (или по $\lambda \geqslant 0,0 \leqslant h<H$ ) с учетом определения многочленов $\Delta(z, l, H)$ приводит к неравенству

$$
\sum_{l=0}^{\infty}\left(\frac{H}{e}\right)^{l} \frac{1}{H ! \lambda} \frac{1}{h !}=\sum_{\lambda=0}^{\infty}\left(\frac{1}{H !}\left(\frac{H}{e}\right)^{H}\right)^{\lambda} \sum_{h=0}^{H-1} \frac{1}{h !}\left(\frac{H}{e}\right)^{h} \leqslant \sum_{\lambda=0}^{\infty}\left(\frac{1}{e}\right)^{\lambda} e^{H / e},
$$

откуда следует вторая строка (8.3).

Докажем техническую оценку, не выделенную в [1] в самостоятельное утверждение. 
Лемма 8.3. Пусть даньц $\mathbf{x}, \mathbf{y} \in \mathbb{C}^{J}, \mathbf{m} \in \mathbb{N}_{0}^{J},\|\mathbf{m}\| \leqslant M \leqslant \mu, \quad \mu \geqslant 1, a$ marже $\gamma_{0} \geqslant\|\mathbf{m}\|+\|\mathbf{x}\|+\|\mathbf{y}\|>0$. Тогда

$$
\mathscr{D}=\sum_{\mathbf{t}} \frac{1}{\mathbf{t} !^{1}}\left|\mathbf{x}^{\mathbf{t}}\right| \cdot|\Delta(\mathbf{y}, \mathbf{m})| \leqslant \frac{1}{M !} \gamma_{0}^{M}, \quad\left(\frac{\gamma_{0}}{\mu}\right)^{\mu-M} \mathscr{D} \leqslant \frac{1.1}{\sqrt{2 \pi \mu}}\left(\frac{e \gamma_{0}}{\mu}\right)^{\mu}
$$

(суммирование ведется по наборам $\mathbf{t} \in \mathbb{N}_{0}^{J},\|\mathbf{t}\|=M-\|\mathbf{m}\| ;\|\cdot\|$ обозначает sum-норму; $\mathbf{1}=(1, \ldots, 1) \in \mathbb{Z}^{J} ;$ обозначения $\mathbf{x}^{\mathbf{t}}, \Delta(\mathbf{y}, \mathbf{m})$ см. в $\left.\S 3\right)$.

В частности, оценка верна при фиксированных $\mathbf{t}=\mathbf{0}$ и $\mathbf{x}=\mathbf{0}$ :

$$
\left(\frac{\gamma_{0}}{\mu}\right)^{\mu-M}|\Delta(\mathbf{y}, \mathbf{m})| \leqslant \frac{1.1}{\sqrt{2 \pi \mu}}\left(\frac{e \gamma_{0}}{\mu}\right)^{\mu}
$$

ДокАЗАТЕЛЬСтво. Заменяя $x_{j}, y_{j}$ на $\left|x_{j}\right|,\left|y_{j}\right|$, можно для краткости считать $\mathbf{x}, \mathbf{y} \geqslant \mathbf{0}$. Тогда, рассматривая суммирование по $\mathbf{n},\|\mathbf{n}\|=\|\mathbf{m}\|$ (среди слагаемых есть и наше $\mathbf{m})$, получаем

$$
\mathscr{D} \leqslant \frac{1}{M !} \sum_{\mathbf{n}, \mathbf{t}} \frac{M !}{\mathbf{n} !^{1} \mathbf{t} !^{1}} \mathbf{x}^{\mathbf{t}}(\mathbf{y}+\mathbf{m})^{\mathbf{n}}=\frac{1}{M !}(\|\mathbf{x}\|+\|\mathbf{y}\|+\|\mathbf{m}\|)^{M}
$$

что дает первое неравенство в (8.4).

Для доказательства второго неравенства (8.4) надо установить, что

$$
f(\mu)=\frac{1}{2} \ln (2 \pi \mu)+M \ln (\mu)-\mu-\ln (M !)<\ln (1.1) .
$$

При $M=0$ легко получаем, что худшее значение $\mu$ равно 1 , и здесь (8.5) выполнено. Дифференцируя функцию $f(\mu)$, получаем, что для $M \geqslant 1$ максимум достигается при $\mu=M+0.5$. В этом случае

$$
\begin{aligned}
f\left(M+\frac{1}{2}\right) & =\frac{1}{2} \ln (2 \pi M)+M \ln (M)-M-\ln (M !)+f_{1}(M), \\
f_{1}(M) & =\left(M+\frac{1}{2}\right) \ln \left(1+\frac{1}{2 M}\right)-\frac{1}{2} .
\end{aligned}
$$

Поскольку функция $f_{1}(M)$ убывает, наименьшее значение она принимает при $M=1$. Кроме того, из неравенства Стирлинга:

$$
x^{x} e^{-x}(2 \pi x)^{1 / 2}<(x) !<x^{x} e^{-x}(2 \pi x)^{1 / 2} e^{1 /(12 x)}, \quad x \in \frac{1}{2} \mathbb{Z}, \quad x>0
$$

получаем, что (8.5) достаточно проверить при $M=1,2$. Непосредственный подсчет подтверждает требуемое неравенство. 


\section{§9. Общий вид вспомогательных функций}

Будем записывать вспомогательные функции в современном виде, пригодном для применения введенных ранее, в (3.1), дифференциальных операторов $\partial_{j}$ и для оценки кратностей нулей (см. $§ 19)$ :

$$
\mathscr{F}_{s}\left(\bar{Y}^{\prime}\right)=\mathscr{F}_{s}\left(Y_{0}, \ldots, Y_{n}\right)=\sum^{(s)} P_{s}\left(Y_{0}, \bar{l}\right) \bar{Y}^{\bar{l}}, \quad s=0,1, \ldots, S,
$$

где суммирование идет по значениям $\bar{l}$ из некоторого набора мультистепеней $\mathscr{L}_{s}$, а $P_{s}\left(Y_{0}, \bar{l}\right)$ - некоторые многочлены от $Y_{0}$. Запишем их в базисе обобщенных биномиальных многочленов

$$
P_{s}(z, \bar{l})=\sum_{l_{0}=0}^{L_{0}} p\left(\bar{l}^{\prime}\right) \Delta\left(2^{S-s} z, l_{0}, H\right)
$$

с неопределенными коэффициентами $p\left(\bar{l}^{\prime}\right)=p\left(l_{0}, \ldots, l_{n}\right) \in \mathbb{K}$ и с некоторым $H$. Тогда

$$
\operatorname{deg}_{Y_{0}} \mathscr{F}_{s}\left(Y_{0}, \ldots, Y_{n}\right) \leqslant L_{0} .
$$

С ростом $s$ наборы мультистепеней будут определенным образом прореживаться, при этом будет

$$
\mathscr{L}_{S} \subseteq 2^{-1} \mathscr{L}_{S-1} \subseteq \cdots \subseteq 2^{1-S} \mathscr{L}_{1} \subseteq 2^{-S} \mathscr{L}_{0}
$$

что позволяет не перенумеровывать $p\left(\bar{l}^{\prime}\right)$ в зависимости от $s$. Подробнее строение множества $\mathscr{L}_{s}$ описано далее, в $\S 10$.

В [1] были использованы несколько иные обозначения. Их связь с вышеуказанными функциями (9.1) следующая:

$$
F_{s}\left(x, \widetilde{m}^{\prime}\right)=\tilde{\partial}^{\prime \tilde{m}^{\prime}} \mathscr{F}_{s}\left(x, \alpha_{1}^{x}, \ldots, \alpha_{n}^{x}\right)=\sum^{(s)} P_{s}^{\left(m_{0}\right)}(x, \bar{l}) \tilde{\chi}^{\widetilde{m}}(\bar{l}) \bar{\alpha}^{\bar{l} x}
$$

Мотивировкой для рассмотрения подобного рода функций и операторов служит следующее соображение. Предполагая малость линейной формы от логарифмов и делая в выражении $\mathscr{F}_{s}\left(z, \alpha_{1}^{z}, \ldots, \alpha_{n}^{z}\right), z \in \mathbb{C}$, замену с небольшим поправочным членом

$$
\alpha_{n}^{l_{n} z} \approx \exp \left(-(\tilde{b} \cdot \ln \tilde{\alpha}) z l_{n} / b_{n}\right)
$$

мы получаем аналитическую функцию

$$
\mathscr{G}_{s}(z)=\sum^{(s)} P_{s}(z, \bar{l}) \prod_{j=1}^{n-1} \alpha_{j}^{\left(l_{j} b_{n}-l_{n} b_{j}\right) z / b_{n}}=\sum^{(s)} P_{s}(z, \bar{l}) \tilde{\alpha}^{\tilde{\chi}(\bar{l}) z / b_{n}} .
$$

При ее дифференцировании и возникают вьшеприведенные выражения. Компоненты в $(9.3)$ будем называть соответственно: $P_{s}(x, \bar{l})$ - полиномиальной частью,

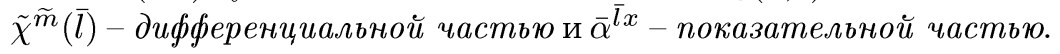


Основная часть доказательства заключается в построении нетривиального набора функций, удовлетворяющих соотношениям

$$
F_{s}\left(x, \tilde{m}^{\prime}\right)=0, \quad x \in \mathscr{X}_{s}, \quad\left\|\widetilde{m}^{\prime}\right\| \leqslant M_{s}, \quad s=0,1, \ldots, S,
$$

для некоторых чисел (кратностей) $M_{s}$ и конечных множеств (нулей) $\mathscr{X}_{s} \subseteq \mathbb{Z}$, которые будут определены в следующих параграфах.

Отметим, какие преобразования можно применять к этим функциям. Показатели $l_{j}, 1 \leqslant j \leqslant n$, могут принимать отрицательные значения. Умножая $\mathscr{F}$ на подходящую степень $\bar{Y} \bar{l}$, мы можем сдвинуть все показатели в положительную область. Это обстоятельство, позволяющее превратить $\mathscr{F}$ в многочлен, потребуется нам в конце доказательства. Степени по $Y_{j}$ будут определяться в зависимости от изменения $l_{j}$. Такое домножение на $\bar{Y} \bar{l}$ не влияет на систему уравнений $(9.5)$.

Далее, и в этом новизна доказательства, показатели $l_{j}$ будут допускать дробные значения, точнее, иметь вид

$$
\bar{l}=\bar{v}_{s}+\bar{\lambda} \in \mathscr{L}_{s}, \quad \bar{\lambda} \in \frac{1}{N} \mathbb{Z}^{n}
$$

Тогда после сдвига на $\bar{v}_{s}$ и замены переменных $\bar{Z}=\bar{Y}^{1 / N}$ получаем целочисленные показатели. Это также понадобится в конце доказательства для перехода к многочленам. При такой замене степень может вырасти в $N$ раз.

Отметим также, что аналитические свойства функций не зависят от арифметической природы векторов $\bar{v}_{s}$, а в алгебраических рассуждениях мы можем вынести соответствующий множитель за скобки, оставляя внутри только корректно определенные алгебраические выражения.

Линейная замена переменной $x$ позволяет преобразовать нули из $(9.5)$, образующие арифметическую прогрессию, к виду $x=0, \ldots, X^{\prime}$, что также оказьвается востребованным в конще доказательства для применения оценки кратностей нулей.

Все эти преобразования несколько влияют на коэффициенты функций, но принципиально не портят систему (9.5).

Приведем уравнения (9.5) к более удобному для решения виду. Заменим в функциях $F_{s}$ из (9.3) дифференциальную часть $\tilde{\chi}(\bar{l})^{\widetilde{m}}$ на $\Delta\left(2^{s} N \tilde{\chi}\left(\bar{l}-\bar{w}_{s}\right), \widetilde{m}\right)$ (которую

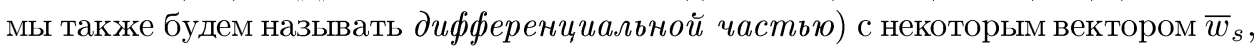
выбор которого определяется соотношениями

$$
\bar{l}-\bar{w}_{s} \in\left(2^{s} N\right)^{-1} \mathbb{Z}^{n}, \quad \bar{l} \in \mathscr{L}_{s}, \quad\left|w_{s, j}\right| \leqslant 2^{-1-s} N^{-1}, \quad 1 \leqslant j \leqslant n .
$$

Затем, умножив $F_{s}$ на $\frac{1}{m_{0} !} \bar{\alpha}^{-\bar{v}_{s} x} d_{0}\left(m_{0}\right) 2^{-(S-s) m_{0}}\left(d_{0}\right.$ из леммы 8.1$)$, получаем

$$
\begin{aligned}
\Phi_{s}\left(x, \widetilde{m}^{\prime}\right)= & \sum \sum_{l_{0}=0}^{(s)} a_{s}\left(\bar{l}^{\prime} ; x, \widetilde{m}^{\prime}\right) p\left(\bar{l}^{\prime}\right), \\
a_{s}\left(\bar{l}^{\prime} ; x, \widetilde{m}^{\prime}\right)= & d_{0}\left(m_{0}\right) 2^{-(S-s) m_{0}} \frac{1}{m_{0} !} \Delta^{\left(m_{0}\right)}\left(2^{S-s} x, l_{0}, H\right) \\
& \times \Delta\left(2^{s} N \tilde{\chi}\left(\bar{l}-\bar{w}_{s}\right), \widetilde{m}\right) \bar{\alpha}^{\bar{\lambda} x} .
\end{aligned}
$$


Согласно лемме 3.1 системы уравнений (9.5) при каждом $s$ эквивалентны соответствуюшим системам:

$$
\Phi_{s}\left(x, \widetilde{m}^{\prime}\right)=0, \quad x \in \mathscr{X}_{s}, \quad\left\|\widetilde{m}^{\prime}\right\| \leqslant M_{s}, \quad s=0,1, \ldots, S .
$$

Соответственно после замены (9.4) вместо функции $\mathscr{G}_{s}(z)$ мы получаем функции вида

$$
\begin{aligned}
\Psi_{s}\left(z, \widetilde{m}^{\prime}\right)= & \sum_{l_{0}=0}^{(s)} \sum_{0}^{L_{0}} p\left(\bar{l}^{\prime}\right) 2^{-(S-s) m_{0}} \frac{1}{m_{0} !} \Delta^{\left(m_{0}\right)}\left(2^{S-s} x, l_{0}, H\right) \\
& \times d_{0}\left(m_{0}\right) \Delta\left(2^{s} N \tilde{\chi}\left(\bar{l}-\bar{w}_{s}\right), \widetilde{m}\right) \tilde{\alpha} \tilde{\chi}(\bar{\lambda}) z / b_{n}
\end{aligned}
$$

\section{§10. Описание набора мультистепеней}

Основным приемом, позволившим в [1] убрать из оценок линейных форм множитель $n^{n}$ для случая сильной независимости, было усовершенствование в выборе набора мультистепеней $\mathscr{L}_{0}$. Этот же прием был использован в более простой ситуации в работе [5]. Устранение второго множителя $n^{n}$ в данной работе обязано еше одному усовершенствованию в конструкции мультистепеней.

Теперь приступим к построению множества $\mathscr{L}_{0}$.

Пусть $\mathbf{a}_{1}, \ldots, \mathbf{a}_{n}$ - векторы в расширенном логарифмическом пространстве $\mathscr{E}_{\varkappa}$, соответствуюшие числам $\alpha_{1}, \ldots, \alpha_{n}$ с выбранными значениями логарифмов (координаты векторов пронумерованы индексами $\sigma \geqslant 1$ ). Тогда числу $\bar{\alpha} \bar{l}$ соответствует вектор $l_{1} \mathbf{a}_{1}+\cdots+l_{n} \mathbf{a}_{n}$ из $\mathscr{E}_{\varkappa}$. Рассмотрим в $\mathscr{E}_{\varkappa}$ решетки

$$
\mathrm{M}_{n}^{\prime}=\left\langle\mathbf{a}_{1}, \ldots, \mathbf{a}_{n}\right\rangle_{\mathbb{Z}}, \quad \mathrm{M}_{n}=\left\langle\mathbf{a}_{1}, \ldots, \mathbf{a}_{n}\right\rangle_{\mathbb{R}} \cap \mathrm{M}_{\varkappa}(\mathbb{K}) .
$$

Решетке $\mathrm{M}_{n}^{\prime}$ отвечает в пространстве мультистепеней $\bar{l}$ решетка $\mathscr{M}_{n}^{\prime}=\mathbb{Z}^{n}$. Этому соответствует обычный выбор множества $\mathscr{L}_{0}$ в предшествуюших работах. Но в расширенном логарифмическом пространстве решетка $\mathrm{M}_{n}$ может содержать и другие точки. Иньми словами, условию $\bar{\alpha}^{\bar{l}} \in \mathbb{K}$ могут удовлетворять не только целые, но и дробные мультистепени. Именно это обстоятельство позволяет перейти без потерь точности к общему случаю. Пусть $\mathscr{M}_{n}$ - решетка в пространстве мультистепеней $\mathbb{R}^{n}$, соответствуюшая решетке $\mathrm{M}_{n}$. Положим

$$
N=\left[\mathrm{M}_{n}: \mathrm{M}_{n}^{\prime}\right]=\left[\mathscr{M}_{n}: \mathscr{M}_{n}^{\prime}\right]
$$

тогда

$$
\operatorname{Vol}_{n} \mathscr{M}_{n}=\frac{1}{N} \operatorname{Vol}_{n} \mathscr{M}_{n}^{\prime}=\frac{1}{N} .
$$

Укажем строение решетки $\mathscr{M}_{n}$, чтобы воспользоваться этим в дальнейшем для описания остальных множеств $\mathscr{L}_{s}$. Выберем в $\mathrm{M}_{n}$ какой-либо базис $\mathbf{e}_{1}, \ldots, \mathbf{e}_{n}$, и пусть $\mathscr{A}$-матрица строк координат (целочисленных) векторов $\mathbf{a}_{i}$ в этом базисе; тогда $N=|\operatorname{det} \mathscr{A}|$, а векторы $\mathbf{e}_{j}$ выражаются через векторы $\mathbf{a}_{i}$ с помошью обратной матрицы $\mathscr{A}^{-1}$, где $\bar{u}_{j}-$ строки матрицы $\mathscr{A}^{-1}$ - состоят из координат векторов 
$\mathbf{e}_{j}$ в базисе $\left\{\mathbf{a}_{i}\right\}$. Так что если $\bar{\mu}=\left(\mu_{1}, \ldots, \mu_{n}\right) \in \mathbb{Z}^{n}$ - координаты какого-либо вектора $\mathbf{v} \in \mathrm{M}_{n}$ в базисе $\left\{\mathbf{e}_{j}\right\}$, то его координаты в базисе $\left\{\mathbf{a}_{i}\right\}$ равны

$$
\bar{\lambda}=\bar{\lambda}(\bar{\mu})=\left(\lambda_{1}, \ldots, \lambda_{n}\right)=\mu_{1} \bar{u}_{1}+\cdots+\mu_{n} \bar{u}_{n}=\bar{\mu} \mathscr{A}^{-1} .
$$

Из определения числа $N$ следует, что $N \bar{\lambda}(\bar{\mu}) \in \mathbb{Z}^{n}$.

Теперь определим область, в которой будут лежать рассматриваемые векторы. Возьмем некоторое $L>0$ и зададим тело $\mathscr{W}$ условиями

$$
\left|w_{j}\right| \leqslant \frac{L}{2 A_{j}}, \quad 1 \leqslant j \leqslant n
$$

Именно индивидуальные границы для каждой координаты позволили Бейкеру в $[14]$ получить оценку линейной формы в зависимости от $\Omega$. Отметим, что с учетом обозначений для $A_{j}$

$$
\sup \{|\bar{w} \cdot \ln \bar{\alpha}|: \bar{w} \in \mathscr{W}\} \leqslant \eta_{0}^{-1} L, \quad \eta_{0}=2 / n .
$$

Основная идея устранения множителя $n^{n}$ в [1] заключается в том, чтобы принудительно уменьшить гранищу для $|\bar{w} \cdot \ln \bar{\alpha}|$. В данной работе мы положим

$$
|\bar{w} \cdot \ln \bar{\alpha}| \leqslant \eta^{-1} L, \quad \eta=e^{1+(n+0.53) / \varkappa},
$$

и определим тело $\mathscr{W}_{0}$ условиями (10.2), (10.3). В [1, лемма 5.1] было показано, что

$$
\operatorname{Vol}_{n} \mathscr{W}_{0} \geqslant\left(\frac{\eta}{\eta_{0}}\right)^{-\varkappa} \operatorname{Vol}_{n} \mathscr{W}=\left(\frac{1}{2} n \eta\right)^{-\varkappa} \frac{1}{\Omega} L^{n}
$$

В [1] вместо $\varkappa$ в показателе был более точньй параметр

$$
\rho=\operatorname{rank}_{\mathbb{C}}\left\{\ln \alpha_{1}, \ldots, \ln \alpha_{n}\right\} \leqslant \varkappa,
$$

которьй мы в данной работе не учитьваем (при $\varkappa=1$ имеем $\rho=1$, поскольку значения логарифмов здесь предполагаются действительными).

Небольшая проблема состоит в том, что если просто брать целочисленные точки, то их количество не обязательно соответствует объему тела. Воспользуемся теоремой Блихфельдта (см. [7, гл. 3, теорема 1]), согласно которой найдется вектор $\bar{v}_{0}$ такой, что

$$
\left|\mathscr{W}_{0} \cap\left(\mathscr{M}_{n}+\bar{v}_{0}\right)\right|>\frac{\operatorname{Vol}_{n} \mathscr{W}_{0}}{\operatorname{Vol}_{n} \mathscr{M}_{n}} \geqslant\left(\frac{1}{2} n \eta\right)^{-\varkappa} \frac{N}{\Omega} L^{n} .
$$

Именно эти точки мы и выберем в качестве набора $\mathscr{L}_{0}$, при этом, отбрасывая в случае необходимости лишние точки, можно считать

$$
\left|\mathscr{L}_{0}\right|=\left[\left(\frac{1}{2} n \eta\right)^{-\varkappa} \frac{N}{\Omega} L^{n}\right]+1 .
$$

Кроме того, можно считать, что $\bar{v}_{0} \in \mathscr{L}_{0} \subseteq \mathscr{W}_{0}$. 
Теперь положим

$$
\begin{aligned}
\bar{v}_{s} & =2^{-s} \bar{v}_{0}, \quad s \geqslant 0, \\
\mathscr{L}_{s} & =2^{-s} \mathscr{L}_{0} \cap\left(\mathscr{M}_{n}+\bar{v}_{s}\right), \quad 0 \leqslant s \leqslant S,
\end{aligned}
$$

и отметим, что при $s=0$ получаем $\mathscr{L}_{0}$ и что $\bar{v}_{s} \in \mathscr{L}_{s} \cap 2^{-s} \mathscr{W}_{0}$, а точки $\mathscr{L}_{s}$ сравнимы по модулю решетки $\mathscr{M}_{n}$. Кроме того, выполнено и условие $(9.2)$, поскольку

$$
2 \mathscr{L}_{s+1} \subseteq 2^{-s} \mathscr{L}_{0} \cap\left(2 \mathscr{M}_{n}+2 \bar{v}_{s+1}\right) \subseteq 2^{-s} \mathscr{L}_{0} \cap\left(\mathscr{M}_{n}+\bar{v}_{s}\right)=\mathscr{L}_{s}
$$

Очевидно, построенные в (10.5) множества согласуются с условиями (9.6). Отметим, что в данной статье мы работаем со сдвинутыми множествами, отдавая предпочтение их центрированности, а не арифметическим свойствам. Это создает определенные удобства и позволяет упростить выкладки. При изучении арифметических выражений множитель $\bar{\alpha} \bar{v}$ выносится за скобки.

\section{§ 11. Схема арифметико-аналитического продолжения}

Доказательства в методах, восходящих к работам Гельфонда, основаны на построении вспомогательной функции, имеющей достаточно большое число нулей с учетом их кратностей (это делается с помощью оценки, обычно называемой леммой Зигеля, см. $\S 13-15)$, а затем с помощью интерполяционной техники и в предположении малости линейной формы соотношение между нулями и их кратностями изменяется (см. §16-18) так, чтобы их число стало больше допустимого (теорема об оценке кратности нулей; $\S 19)$. Полученное противоречие нашему предположению и доказывает оценку линейной формы.

В схеме Бейкера увеличение числа нулей происходит за счет уменьшения кратности. В то же время увеличение числа нулей ухудшает оценку линейной формы, поэтому проводить его надо максимально экономно.

Важным нововведением в работе [15] было использование теории Куммера и принципа деления аргумента, позволяющего расщеплять вспомогательную функцию на части, что равноценно уменьшению ее степени. Это позволяет применять теорему об оценке кратностей нулей, уменьшая количество итераций. В [4], [1] и в данной работе на каждом шаге происходит уменьшение степени в два раза. Это позволяет наиболее экономно проводить деление аргумента.

Арифметико-аналитическоепродолжение будет организовано индуктивно по $s-$ числу делений аргумента. Основание индукции $(s=0)$ будет построено в 15 . Шаг индукции будет состоять в применении принципа деления аргумента (см. $\S 18$ ) для получения равенств (9.5), пока степень вспомогательной функции не понизится до требуемой величины.

Однако прежде чем применять деление аргумента, необходимо сначала увеличить количество нулей примерно в $2^{n}$ раз (также за счет некоторой потери кратности). В [1] была предложена довольно экономная, но технически трудная схема плавного понижения кратности с ростом $x$ - количества узлов интерполящии. В данной работе этот этап доказательства упрощен, что ухудшает оценку на множитель порядка $e^{n}$ и в абсолютной константе. Процесс предварительного размножения нулей разбит на $n$ частей и также происходит индуктивно по $\nu=0, \ldots, n$ при 
фиксированном $s$. В качестве основания $(\nu=0)$ берутся нули с кратностями, полученными на очередном шаге по $s$. Далее число нулей каждый раз удваивается, а кратность понижается на $T_{s}$; еше $T_{s}$ кратностей теряется при делении аргумента. Такая схема позволит получить константы, соответствуюшие результату [3] .

Пусть $\varepsilon_{0}>0$ - некоторая константа, а $C_{0} \xi \omega$ - некоторое (не маленькое) выражение, которые будут определены в следующем параграфе. Положим

$$
\begin{gathered}
c_{1}=2(n+1)\left(1+\varepsilon_{0}\right), \quad M_{0}=\left[C_{0} \xi \omega\right], \quad T_{s}=\left[\frac{2^{-s} C_{0} \xi \omega}{c_{1}}\right], \\
M_{s, \nu}=M_{s}-\nu T_{s}, \quad \nu=0, \ldots, n, \\
M_{s+1}=M_{s}-(n+1) T_{s}, \quad s=0, \ldots, S .
\end{gathered}
$$

Оценим кратность $M_{S, n}$ :

$$
\begin{aligned}
M_{S, n} & =M_{0}-\sum_{s=0}^{S}(n+1) T_{s}+T_{S} \\
& \geqslant C_{0} \xi \omega+T_{S}-1-\sum_{s}^{*} 2^{-s} \frac{C_{0} \xi \omega}{2\left(1+\varepsilon_{0}\right)} \geqslant \frac{\varepsilon_{0} C_{0} \xi \omega}{1+\varepsilon_{0}}
\end{aligned}
$$

(в последней сумме $\sum^{(*)}$ берутся слагаемые по $s$, большие, чем $n+1$, поэтому 1 можно заменить остатком геометрической прогрессии). Параметр $\varepsilon_{0}$ позволяет регулировать остающуюся кратность.

Теперь опишем строение наборов узлов интерполяции. Выбирая некоторое (большое) значение $G>0$, положим

$$
X_{s}=\left\lceil\frac{2 D G}{T_{s}+1}\right\rceil, \quad s \geqslant 0
$$

$(\lceil x\rceil$ означает наименьшее целое число $\geqslant x)$.

Наконец, определим множества $\mathscr{X}_{s, \nu}$ для $(9.5)$ условиями

$$
\begin{aligned}
& \mathscr{X}_{0,0}=\mathscr{X}_{0}=\left\{0, \pm 1, \ldots, \pm X_{0}\right\}, \\
& \mathscr{X}_{s, 0}=\mathscr{X}_{s}=\left\{ \pm 1, \pm 3, \ldots, \pm\left(2 X_{s}-1\right)\right\}, \quad s>0, \\
& \mathscr{X}_{s, \nu}=\left\{0, \pm 1, \ldots, \pm 2^{\nu} X_{s}\right\}, \quad 1 \leqslant \nu \leqslant n, \quad s \geqslant 0,
\end{aligned}
$$

Такой способ задания $T_{s}, \mathscr{X}_{s, \nu}$ обеспечивает выполнение двустороннего неравенства

$$
2^{\nu+2} D G \leqslant\left|\mathscr{X}_{s, \nu}\right|\left(T_{s}+1\right) \leqslant 2^{\nu}\left(4 D G+3 T_{s}+3\right) .
$$

Таким образом, мы будем доказывать равенства, включающие при $\nu=0$ условия (9.5):

$$
F_{s}\left(x, \widetilde{m}^{\prime}\right)=0, \quad x \in \mathscr{X}_{s, \nu}, \quad\left\|\widetilde{m}^{\prime}\right\| \leqslant M_{s, \nu}
$$

с помошью двойной индукции, переходя от $(s, \nu)$ к $(s, \nu+1)$ при $\nu<n$ (см. $\S 17)$ и от $(s, n)$ к $(s+1,0)$ (см. $\S 18)$, теряя каждый раз кратность $T_{s}$. В конце доказательства нам понадобятся только условия (11.6) при $(s, \nu)=(S, n)$. 
Следует заметить, что устранение индивидуальных нижних оценок для $A_{j}$ осуществлено в [1] именно благодаря аккуратной организации арифметико-аналитического продолжения. Хотя все зафиксировано в обозначениях, но рассуждения содержат некоторые тонкие моменты. Здесь мы приводим более развернутые комментарии.

Как уже отмечалось, вспомогательную функцию можно преобразовать к многочлену, имеющему степени $N L / A_{j}$ по переменным $Y_{j}$. В обычной ситуации деление на $A_{j}$ уменьшает степень и упрощает рассуждения. Если же числа $A_{j}$ малы, то степень увеличивается. Тем же недостатком обладает и новый множитель $N$. Это препятствует применению теорем об оценках кратностей нулей. В обычной схеме арифметико-аналитического продолжения это создает непреодолимые трудности, но деление аргумента позволяет, сделав несколько дополнительных шагов индукции по $s$, каждый раз уменьшаюших степень вдвое, удалить этот лишний множитель.

Кроме того, в рассуждениях есть еще один тонкий момент. Ключевую роль в доказательстве и в получаемой оценке играет степень интерполяционного многочлена $\left|\mathscr{X}_{s, \nu}\right|\left(T_{s}+1\right)($ см. $\S 16)$. Из (11.3) видно, что эта степень на каждом шаге почти не меняется, а из (11.1) - что при $T_{s} \gg 1$ (в предшествуюших работах при всех $s$ было именно так) кратность уменьшается примерно в два раза, соответственно число точек удваивается. Теперь же возможен случай $T_{s}=0$, требуюший иного подхода: как только мы получим $T_{s}=0$, то далее $\left|\mathscr{X}_{s, \nu}\right|$ не меняется (увеличение $\left|\mathscr{X}_{s, \nu}\right|$ вело бы к резкому ухудшению оценки линейной формы от логарифмов), соответственно сохраняется степень интерполяционного многочлена. Именно это отражено в формулах (11.3), (11.4).

\section{§ 12. Выбор параметров}

В данном параграфе приводятся формульные значения параметров, необходимых для доказательства основной теоремы, но не участвуюших в ее формулировке. Смысл этих параметров был разъяснен в предыдущих параграфах. Для полноты и компактности напомним некоторые из ранее приведенных формул. Также проведем предварительную оценку указанных выражений.

Как отмечалось, далее можно считать $n \geqslant 2$.

В расширенном логарифмическом пространстве рассматриваются линейно независимые векторы $\mathbf{v}_{j}=\mathbf{v}\left(\alpha_{j}\right)$, соответствуюшие выбранным значениям $\ln \alpha_{j}$. В предложении 5.1 были приведены выражения

$$
\mathrm{M}_{n}=\mathrm{M}_{\varkappa}(\mathbb{K}) \cap\left\langle\mathbf{v}_{1}, \ldots, \mathbf{v}_{n}\right\rangle_{\mathbb{R}}, \quad \mathrm{M}_{n}^{\prime}=\left\langle\mathbf{v}_{1}, \ldots, \mathbf{v}_{n}\right\rangle_{\mathbb{Z}}, \quad N=\left[\mathrm{M}_{n}: \mathrm{M}_{n}^{\prime}\right],
$$

удовлетворяюшие неравенствам (5.1), (5.2).

Введем следующие параметры:

$$
\begin{aligned}
\eta & =e^{1+(n+0.53) / \varkappa}, \quad \xi=\frac{8}{n !}(n+2.1)(2(n+1))^{n+1}\left(\frac{1}{2} n \eta\right)^{\varkappa}, \\
\varepsilon_{0} & =3 n^{3} / \xi, \quad \varepsilon_{1}=\xi_{0}(n) /(2 \xi), \quad \varepsilon_{2}=(4(n+1)(2.25 n+1.5 \ln n+13))^{-1} .
\end{aligned}
$$

Положим

$$
C_{0}=\ln \left(e^{4.4 n+7} n^{5.5} N D^{2} \ln (e D)\right), \quad W_{0}=\ln (1.5 e B N D \ln (e D))
$$


(по сравнению с $(2.2)$ в $C_{0}, W_{0}$ добавлено $N$, так что $C_{0}, W_{0}$ из $(2.2)$ получаются, если положить здесь $N=1)$.

Пусть, далее,

$$
\gamma=\frac{C_{0}(1) W_{0}(1)}{C_{0}(N) W_{0}(N)} N, \quad \omega=\frac{\gamma}{N} D \Omega=\frac{\gamma}{N} D A_{1} \ldots A_{n} .
$$

Отметим, что такой выбор числа $\gamma$ дает

$$
C_{0}(N) W_{0}(N) \omega(N, \gamma)=C_{0}(1) W_{0}(1) \omega(1,1)
$$

и мы можем просто писать $C_{0} W_{0} \omega$, устраняя параметры $N, \gamma$ из оценок линейных форм. Так что в дальнейшем мы будем считать $C_{0}, W_{0}$ зависяшими от параметра $N$, как это определено в данном параграфе, но обычно в записи $C_{0}, W_{0}$ будем его опускать.

Пусть

$$
S=\left\lceil\log _{2}\left(0.5 e^{-3} n^{2} \xi N D \ln (e D)\right)\right\rceil, \quad G=C_{0} W_{0} \xi \omega, \quad c_{1}=2(n+1)\left(1+\varepsilon_{0}\right)
$$

(см. (11.1)),

$$
M_{0}=\left[C_{0} \xi \omega\right], \quad T_{s}=\left[\frac{1}{c_{1}} 2^{-s} C_{0} \xi \omega\right], \quad X_{s}=\left\lceil\frac{2 D G}{T_{s}+1}\right\rceil, \quad s \geqslant 0
$$

(см. (11.3)),

$$
L=\frac{1}{c_{1}} C_{0} \xi \omega, \quad L_{0}=\left[W_{0} \xi \omega\right], \quad H=[0.96 \ln (1.5 B N D \ln (e D))]
$$

(см. $\S 8$ ), а параметры $K, c$ в леммах $8.1,8.2$ будут равны $2^{S-s}$.

Укажем простейшие оценки введенных вьше параметров. Поскольку выражение $C_{0}(N) W_{0}(N)$ с ростом $N$, очевидно, растет, а $C_{0}(N) W_{0}(N) / N$ убывает, то имеем $1 \leqslant \gamma \leqslant N$. Выбор $\varepsilon_{1}$ дает ввиду (5.1) неравенство

$$
\varepsilon_{1} \xi \omega \min \left\{C_{0}, W_{0}\right\} \geqslant 1 .
$$

Из (6.2) видно, что можно считать $W_{0}>C_{0} / 2$, а для $\varepsilon_{2}$ мы имеем $2 c_{1} W_{0} \varepsilon_{2}>1$.

Таким образом, $\varepsilon_{1}, \varepsilon_{2}$ являются оценками относительной погрешности при замене $M_{0}, L_{0}, X_{0}$ на их правые части из соответствуюших формул без округления.

Проведем также предварительную оценку параметров, чтобы в дальнейшем можно было упрошать рассматриваемые выражения. С ростом $n$ имеем

$$
\begin{gathered}
\xi=\left(2 e^{2}\right)^{n} n^{\varkappa+1.5} O(1)>1.2 \cdot 10^{5}, \quad M_{0} \geqslant 2 \xi / \xi_{0}(n)>1.3 \cdot 10^{4} \\
N L / A_{j} \geqslant 2 \xi /\left(2(n+1) \xi_{0}(n-1)\right)>2.8 \cdot 10^{4} \\
\varepsilon_{0}=\left(2 e^{2}\right)^{-n} n^{1.5-\varkappa} O(1)<2 \cdot 10^{-4} \\
\varepsilon_{1}=\left(2 e^{2} / 3.1\right)^{-n} n^{-1.5} O(1)<10^{-4} \\
\varepsilon_{2}=O\left(n^{-2}\right)<5 \cdot 10^{-3} .
\end{gathered}
$$


Таким образом, параметры $\varepsilon_{0}, \varepsilon_{1}$ экспоненциально малы, а $M_{0}, L_{0}, L, N L / A_{j}$ экспоненциально растут с ростом $n$. Мальм оказывается также параметр $\varepsilon_{2}$. Экспоненциальный рост $M_{0}, L_{0}, L, N L / A_{j}$ ухудшает оценку линейной формы, но упрощает доказательство, поскольку позволяет взять значения параметров $\varepsilon_{i}$ малыми. Сразу отметим, что вклад параметров $\varepsilon_{i}$ во всех последуюших оценках будет лиш увеличивать множители не более чем на 0.1 ; так, например, в дальнейшем мы используем округления:

$$
\begin{gathered}
2(n+2)\left(\frac{2 n+1}{\varkappa}+2\right)>e^{0.53}(n+2.1)\left(\frac{2 n+1}{\varkappa}+2+\frac{1}{\eta}+n \varepsilon_{1}\right)\left(1+2 \varepsilon_{2}\right), \\
n+2.1>\left(1+2 \varepsilon_{2}\right)\left(\left(1+\varepsilon_{0}\right)\left(1+n \varepsilon_{1}\right)\right)^{n+1}\left(n+2+2 n \varepsilon_{2}+3 n \varepsilon_{1}\right),
\end{gathered}
$$

проверяемые непосредственно при малых $n$ и верные для больших $n$ вследствие асимптотического поведения $\varepsilon_{i}$.

На шаге индукции $(s, \nu)$ комплексная переменная $z$ будет меняться в диапазоне $|z| \leqslant R_{s, \nu}$, где

$$
R_{s, \nu}=2^{s} \eta\left|\mathscr{X}_{s, \nu}\right|\left(T_{s}+1\right) / L .
$$

Для линейной формы предполагаем выполненной оценку

$$
\ln |\Lambda|<-2^{n+1}\left(\frac{2 n+1}{\varkappa}+2+\frac{1}{\eta}+n \varepsilon_{1}\right) \xi D \omega C_{0} W_{0},
$$

ввиду (12.1) противоположную (2.3), так что для доказательства теоремы 2.1 достаточно привести (12.2) к противоречию.

На примере верхней оценки $M_{0}$ покажем, как мы будем оценивать различные выражения. Для этого расщепим формулу для $M_{0}$ на две составляющие: часть, оцениваемую числом $\exp \left(a C_{0}\right)$ с соответствуюшим значением $a$ за счет выбора $C_{0}$, и часть $x$, включающую все остальное, для которой применим неравенство $x \leqslant e^{x / e}$. В итоге с учетом (5.1) и формулы для $\varepsilon_{1}$ имеем

$$
M_{0}+n \leqslant\left(\left(1+n \varepsilon_{1}\right) \xi\right)\left(C_{0} \omega\right) \leqslant e^{0.7 C_{0}} e^{C_{0} \omega / e} \leqslant e^{0.7 C_{0} \varepsilon_{1} \xi \omega W_{0}} e^{C_{0} \omega / e} \leqslant e^{\varepsilon_{1} G} .
$$

Аналогичньм образом оцениваются сверху $L_{0}, L, L / A_{j}, G, X_{0}, 2^{S}$ и другие подобные выражения.

Наконец, мы можем считать $\mathbb{K}=\mathbb{Q}\left(\alpha_{1}, \ldots, \alpha_{n}\right)$, так как при увеличении степени поля оценка линейной формы ухудшается.

\section{§13. Лемма Зигеля}

В теории чисел результаты о том, что неполная система линейных однородных уравнений имеет ненулевое решение со сравнительно небольшими значениями неизвестных, обычно называют леммой Зигеля. В случае алгебраических линейных уравнений современная оценка решений была получена в [15]. Мы используем ее уточненный вариант [8], учитьваюший весовые коэффициенты. Для этого предварительно введем некоторые обозначения.

Для изучения векторов и линейных форм над $\mathbb{K}$ в [8] были введены понятия соответствуюших (евклидовых) длин. 
Пусть дан набор положительных чисел (весовых коэффициентов)

$$
\mathscr{Q}=\left\{q_{j, \sigma}: 1 \leqslant j \leqslant J ; \sigma \geqslant 1\right\} .
$$

Будем считать, что $q_{j, \sigma}=q_{j, \tau}$ для комплексно-сопряженных $\sigma, \tau$, а при $\sigma>D$ выполняется $q_{j, \sigma}=\left(\operatorname{Norm~p}_{\sigma}\right)^{l_{j, \sigma}}$ с некоторыми $l_{j, \sigma} \in \mathbb{Z}$. Кроме того, $q_{j, \sigma}=1$ для почти всех $\sigma$.

Через $\mathscr{Q}^{\prime}$ будем обозначать набор обратных к $\mathscr{Q}$ величин.

Определим локальные и абсолютную длины вектора

$$
\mathbf{a}=\left(a_{1}, \ldots, a_{J}\right) \in \mathbb{K}^{J}
$$

с весовыми коэффициентами $\mathscr{Q}^{\prime}$ с помощью равенств

$$
\begin{aligned}
\|\mathbf{a}\|_{\mathscr{Q}^{\prime}}^{(\sigma)}= & \left(\sum_{j=1}^{J}\left|a_{j}\right|_{\sigma}^{2} / q_{j, \sigma}^{2}\right)^{1 / 2}, \quad 1 \leqslant \sigma \leqslant D, \\
\|\mathbf{a}\|_{\mathscr{Q}^{\prime}}^{(\sigma)}= & \max \left\{\left|a_{j}\right|_{\sigma} / q_{j, \sigma}: 1 \leqslant j \leqslant J\right\}, \quad \sigma>D, \\
& \|\mathbf{a}\|_{\mathscr{Q}^{\prime}}=\left(\prod_{\sigma=1}^{\infty}\|\mathbf{a}\|_{\mathcal{Q}^{\prime}}^{(\sigma)}\right)^{1 / D}
\end{aligned}
$$

(мы пишем индекс $\mathscr{Q}^{\prime}$, чтобы согласовать форму вхождения весовых коэффициентов в норму с формулой (4.3) и с обозначениями работы [8]). Обозначение $\|\cdot\|_{\mathcal{Q}^{\prime}}$ будет зарезервировано именно для вьшеуказанных норм.

Лемма 13.1 [8, теорема 3]. Пусть даньь векторы $\mathbf{a}_{i} \in \mathbb{K}^{J}, \quad 1 \leqslant i \leqslant I, u$ набор $\mathscr{Q}$ весовых коэффичиентов. Положим

$$
q_{j}=\left(\prod_{\sigma=1}^{\infty} q_{j, \sigma}\right)^{1 / D}, \quad 1 \leqslant j \leqslant J, \quad q=\left(q_{1} \ldots q_{J}\right)^{1 / J} .
$$

Тогда система линейных уравнений ранга $r \leqslant I<J$

$$
\mathbf{a}_{i} \cdot \mathbf{p}=0, \quad 1 \leqslant i \leqslant I,
$$

имеет ненулевое решение $\mathbf{p} \in \mathbb{K}^{J}$, удовлетворяющее условию

$$
\|\mathbf{p}\|_{\mathscr{Q}} \leqslant \gamma\left(q^{-J} \prod_{i=1}^{I} \max \left\{q,\left\|\mathbf{a}_{i}\right\|_{\mathcal{Q}^{\prime}}\right\}\right)^{1 /(J-I)},
$$

где в случае $r=0$ или $r=I=J-1$ можно взять $\gamma=1$, при $r=I<J-1$ можно взять

$$
\gamma=2 \rho_{(J-I) D}|\Delta(\mathbb{K})|^{1 /(2 D)} / \sqrt{D},
$$

здесь $\rho_{m}$ обозначает радиус т-мерного шара единичного оббема, а $\Delta(\mathbb{K})$ дискриминант поля $\mathbb{K}$, и всегда можно взять

$$
\gamma=\sqrt{J}|\Delta(\mathbb{K})|^{1 /(2 D)} .
$$


ЗАмЕчАНИЕ 13.1. В [8] прототип формулы (13.2) содержал коэффициент 0.5 для случая $J>4$. В подтверждение этому в доказательстве приводилось неравенство, основанное на формуле Стирлинга для факториала, присутствующего в $\rho$, верное асимптотически и проверяемое численно для малых $J$. Повторная проверка показала, что 0.5 можно ставить только при $J>68$, а выражение (13.2) (без множителя 0.5) верно для любых $J$.

ЗАмЕчАниЕ 13.2. Хотя в данной работе это не понадобится, отметим, что в [8] при доказательстве леммы Зигеля были оценены также и локальные длины решения $\mathbf{p}$. При $\sigma>D$ получалось $\|\mathbf{p}\|_{\mathscr{Q}}^{(\sigma)} \leqslant 1$. Для $q_{j, \sigma}=1, \sigma>D$, это означает, что $\mathbf{p}$ имеет целые алгебраические коэффициенты.

Для изучения компонент рассматриваемых в дальнейшем векторов приведем простую лемму с учетом понятий, введенных в $§ 5$.

ЛЕмма 13.2. Если компоненты некоторого набора $\left\{\gamma_{j}\right\}$ разлагаются в поле $\mathbb{K}$ на сомножители $\gamma_{j}=\delta_{j} \varepsilon_{j}$, то

$$
\begin{aligned}
& \left\|\left\{\gamma_{j}\right\}\right\|_{\mathscr{Q}^{\prime}}^{(\sigma)} \leqslant \exp \left(\mathrm{h}^{(\sigma)}\left(\left\{\delta_{j}\right\}\right)\right)\left\|\left\{\varepsilon_{j}\right\}\right\|_{\mathscr{Q}^{\prime}}^{(\sigma)}, \quad \sigma \geqslant 1, \\
& \left\|\left\{\gamma_{j}\right\}\right\|_{\mathscr{Q}^{\prime}} \leqslant \exp \left(\mathrm{h}\left(\left\{\delta_{j}\right\}\right)\right)\left\|\left\{\varepsilon_{j}\right\}\right\|_{\mathscr{Q}^{\prime}}
\end{aligned}
$$

ДокАЗАТЕЛЬСтво. Первое неравенство означает, что из рассматриваемых норм можно выносить за скобки максимальный элемент, отвечающий $\mathrm{h}^{(\sigma)}\left(\left\{\delta_{j}\right\}\right)$. Второе неравенство получается путем взятия произведения по $\sigma$ первых неравенств.

Эта лемма позволяет изучать нормы векторов, расщепляя их координаты на компоненты, причем ясно, что можно выделять не только два, но и большее число сомножителей.

Напомним также, что для $\mathbf{a}, \mathbf{p} \in \mathbb{K}^{J}$ вьполнено

$$
|\mathbf{a} \cdot \mathbf{p}|_{\sigma} \leqslant\|\mathbf{a}\|_{\mathscr{Q}^{\prime}}^{(\sigma)} \cdot\|\mathbf{p}\|_{\mathscr{Q}}^{(\sigma)} .
$$

\section{§14. Дискриминант}

Для оценки модуля дискриминанта $\Delta(\mathbb{K})$, участвуюшего в лемме Зигеля, понадобится следуюшее утверждение.

ЛЕмма 14.1 [1, лемма 6.2]. Пусть даны алъебраические $\alpha_{1}, \ldots, \alpha_{n}, \quad \mathbb{K}=$ $\mathbb{Q}\left(\alpha_{1}, \ldots, \alpha_{n}\right), \quad D=[\mathbb{K}: \mathbb{Q}]$. Тогда

$$
\ln |\Delta(\mathbb{K})| \leqslant D \ln D+2 D(D-1) \max \left\{\mathrm{h}\left(\alpha_{j}\right): 1 \leqslant j \leqslant n\right\} .
$$

Отметим, что результаты статьи [1] были доложены на конференциях в Венгрии (г. Эгер, 29.07-2.08.1996 г.) и в Беларуси (г. Минск, 1-8.09.1996 г.). Эта же лемма приводится в [17] со ссылкой на письмо Массера Вюстхольцу, датированное 08.12 .1990 г.

В оценке $|\Delta(\mathbb{K})|$ отсутствует параметр $N$, что не только препятствует его включению в формулировку теорем, но и создает определенную трудность в доказательстве, которая будет обойдена в данном параграфе и для чего ранее, в 112 , был введен параметр $\gamma$. 
ЛЕмма 14.2. В принятых обозначениях имеет место оченка

$$
\frac{1}{2 D} \ln |\Delta(\mathbb{K})| \leqslant 0.3 \varepsilon_{1} G .
$$

ДокаЗАТЕЛЬСтво. С учетом $(5.1),(5.2)$, оценок для $C_{0}, W_{0}$ и определения $\varepsilon_{1}$ имеем

$$
\begin{aligned}
0.3 \varepsilon_{1} G & =0.3 \varepsilon_{1} \xi C_{0}(N) W_{0}(N) \omega(N, \gamma)=0.3 \varepsilon_{1} \xi C_{0}(1) W_{0}(1) \omega(1,1) \\
& \geqslant(0.25+0.05) \frac{1}{2} 3.1^{n} n^{\varkappa} \frac{D}{\varkappa} \Omega C_{0} W_{0} \geqslant \frac{1}{2} \ln D+\max A_{j} .
\end{aligned}
$$

Теперь лемма сразу получается из леммы 14.1.

В заключение параграфа заметим, что техника доказательства оценок линейных форм от логарифмов с помошью интерполяционных определителей (см. [18]) не использует дискриминанта $\Delta(\mathbb{K})$ и поэтому, по-видимому, позволит улучшить оценки в данном аспекте.

\section{$\S 15$. Построение исходной вспомогательной функции}

Основной целью данного параграфа является построение с помощью леммы Зигеля ненулевого набора небольших коэффициентов $p\left(\bar{l}^{\prime}\right) \in \mathbb{K}$ для решения системы (9.9) при $s=0$ с оценкой полученного решения.

В этой системе индексу переменных $p\left(\bar{l}^{\prime}\right)$ соответствует мультииндекс $\bar{l}^{\prime}=\left(l_{0}, \bar{l}\right)$ $=\left(l_{0}, l_{1}, \ldots, l_{n}\right)$, где $0 \leqslant l_{0} \leqslant L_{0}$, а $\bar{l} \in \mathscr{L}_{0}$. Количество переменных при $s=0$ равно $J=\left(L_{0}+1\right)\left|\mathscr{L}_{0}\right|$.

Уравнениям соответствует набор $\left(x, \widetilde{m}^{\prime}\right)=\left(x, m_{0}, \ldots, m_{n-1}\right)$, где $x \in \mathscr{X}_{0}$, $\left\|\widetilde{m}^{\prime}\right\| \leqslant M_{0}$. Количество уравнений (9.9) при $s=0$ равно

$$
I=\left(2 X_{0}+1\right)\left(\begin{array}{c}
M_{0}+n \\
n
\end{array}\right)
$$

Набор коэффициентов $a_{s}\left(\bar{l}^{\prime} ; x, \widetilde{m}^{\prime}\right)$ из $(9.8)$ будем обозначать как вектор $\mathbf{a}_{s}\left(x, \widetilde{m}^{\prime}\right)$. Кроме того, зададим для леммы 13.1 весовые коэффициенты по формуле

$$
\begin{aligned}
& q_{\sigma}\left(\bar{l}^{\prime}\right)=q\left(\bar{l}^{\prime}\right)=e^{C_{0}\left(L_{0}-l_{0}\right)}, \quad 1 \leqslant \sigma \leqslant D, \\
& q_{\sigma}\left(\bar{l}^{\prime}\right)=1, \quad \sigma>D .
\end{aligned}
$$

Тогда параметр $q$ из леммы 13.1 равен

$$
q=\exp \left(\sum_{l_{0}=0}^{L_{0}} \sum^{(0)} C_{0} \frac{L_{0}-l_{0}}{J}\right)=\exp \left(C_{0} \sum_{l_{0}=0}^{L_{0}} \frac{L_{0}-l_{0}}{L_{0}+1}\right)=e^{C_{0} L_{0} / 2} .
$$

Проделаем еще предварительную работу, необходимую также и для следующих параграфов. Мы будем применять лемму 8.3 , взяв

$$
\mu=C_{0} \xi \omega, \quad \gamma_{0}=C_{0} \xi \omega B N 1.5 D \ln (e D),
$$


и векторы $\mathbf{x}, \mathbf{y}$, имеюшие координаты

$$
\begin{aligned}
& x_{j}=\frac{1}{b_{n}}\left(b_{n} l_{j}-b_{j} l_{n}\right) \ln \alpha_{j} \in \mathbb{C}, \quad 1 \leqslant j \leqslant n-1, \\
& y_{j}=2^{s} N\left(b_{n}\left(l_{j}-w_{s, j}\right)-b_{j}\left(l_{n}-w_{s, n}\right)\right) \in \mathbb{Z}, \quad 1 \leqslant j \leqslant n-1 .
\end{aligned}
$$

Оценим $\|\mathbf{x}\|$, принимая во внимание обозначения для $A_{j}, B$, а также условия (10.2). Имеем

$$
\left|x_{j}\right| \leqslant \frac{1}{\left|b_{n}\right|}\left(\left|b_{n} l_{j}\right|+\left|b_{j} l_{n}\right|\right)\left|\ln \alpha_{j}\right| \leqslant \frac{1}{2} L(1+B)
$$

Для оценки $\|\mathbf{y}\|$ надо также воспользоваться (9.7):

$$
\begin{aligned}
& \left|b_{n} 2^{s} N\left(l_{j}-w_{s, j}\right)\right| \leqslant \frac{1}{2}\left|b_{n}\right|\left(\frac{N L}{A_{j}}+1\right) \leqslant \frac{B L N}{2 A_{j}}\left(1+\frac{1}{N L / A_{j}}\right) \\
& \left|b_{j} 2^{s} N\left(l_{n}-w_{s, n}\right)\right| \leqslant \frac{1}{2}\left|b_{j}\right|\left(\frac{N L}{A_{n}}+1\right) \leqslant \frac{B L N}{2 A_{j}}\left(1+\frac{1}{N L / A_{n}}\right),
\end{aligned}
$$

и с учетом (5.3) имеем

$$
\|\mathbf{x}\|+\|\mathbf{y}\| \leqslant(n-1) B N L\left(\frac{1}{1.5}+\frac{1}{1.5 B}+1+\max _{j} \frac{1}{N L / A_{j}}\right) 1.5 D \ln (e D) .
$$

Теперь, учитывая величины $B, L, N L / A_{n}$, а также формулы для $M_{0}, L$ из $\oint 12$ и $\mu, \gamma_{0}$ из $(15.3)$, получаем

$$
M_{0}+\|\mathbf{x}\|+\|\mathbf{y}\| \leqslant B N C_{0} \xi \omega 1.5 D \ln (e D)=\gamma_{0} .
$$

Рассмотрим знаменатель $d_{0}\left(m_{0}\right)$, определенный в лемме 8.1. Здесь $m_{0} \leqslant M_{0}-$ $M, M=\|\tilde{m}\|$, и из (8.2) и определения чисел $H, \gamma_{0}$ имеем

$$
d_{0}\left(m_{0}\right) \leqslant e^{1.04 H(\mu-M)} \leqslant\left(\gamma_{0} / \mu\right)^{\mu-M}
$$

и также

$$
d_{0}\left(m_{0}\right)(M+\|\mathbf{x}\|+\|\mathbf{y}\|)^{M} \frac{1}{M !} \leqslant \frac{1.1}{\sqrt{2 \pi \mu}}\left(\frac{e \gamma_{0}}{\mu}\right)^{\mu} \leqslant 0.01 e^{G}
$$

что вместе с (8.4) позволяет получать необходимые оценки.

ЛЕмма 15.1. При $s \leqslant S,\left\|\tilde{m}^{\prime}\right\| \leqslant M_{0}, \quad x \in 2^{s-S} \mathbb{Z},|x| \leqslant R_{s, \nu}$, имеем

$$
\left\|\mathbf{a}_{s}\left(x, \widetilde{m}^{\prime}\right)\right\|_{\mathscr{Q}^{\prime}} \leqslant 0.02 \exp \left(\frac{1}{D} 2^{-s} L n|x|+2 G+n \varepsilon_{1} G\right) .
$$


ДокАЗАТЕЛЬСТво. Будем оценивать вклад сомножителей, входящих в компоненты векторов а, по отдельности.

Для оценки вклада показательной части $\bar{\alpha} \bar{\lambda} x, \bar{\lambda} \in \mathscr{L}_{s}-\bar{v}_{s} \subseteq \mathscr{M}_{n}$, учтем способ построения в $\S 10$ множеств $\mathscr{L}_{s}, \mathscr{W}_{0}(L)$. Тогда, принимая во внимание обозначения $\S 5$ и лемму 5.3, имеем

$$
\mathrm{h}^{(\sigma)}\left\{\bar{\alpha}, x\left(\mathscr{L}_{s}-\bar{v}_{s}\right)\right\} \leqslant \sum_{j=1}^{n} \max _{ \pm}\left\{x\left( \pm \frac{2^{-s} L}{2 A_{j}}-v_{s, j}\right) \ln \left|\alpha_{j}\right|_{\sigma}\right\}
$$

Возьмем произведение по всем $\sigma \geqslant 1$ и извлечем корень степени $D$, чтобы получить $\|\mathbf{a}\|_{Q^{\prime}}$. С учетом определения (2.1) чисел $A_{j}$ в первом слагаемом мы получим $2^{-s} L n|x| / D$, а слагаемое $v_{s, j} \ln \left|\alpha_{j}\right|_{\sigma}$ при суммировании по $\sigma$ исчезнет вследствие формулы произведения.

При анализе дифференциальной части $\Delta\left(2^{s} N \tilde{\chi}\left(\bar{l}-\bar{w}_{s}\right), \widetilde{m}\right)$ отметим, что первый аргумент целочисленный (ради этого мы заменили сдвиг на $\bar{v}_{s}$ сдвигом на $\bar{w}_{s}$ и домножили все на $2^{s} N$ ). В результате и биномиальное выражение будет целочисленным (в этом и также в делении на $m$ ! заключается смысл перехода от степенных к биномиальным многочленам). Применение леммы 8.3 и (15.4) тогда дает

$$
d_{0}\left(m_{0}\right)\left|\Delta\left(2^{s} N \tilde{\chi}\left(\bar{l}-\bar{w}_{s}\right), \widetilde{m}\right)\right| \leqslant 0.01 e^{G} .
$$

Оценку полиномиальной части проведем совместно с весовым коэффициентом $q\left(\bar{l}^{\prime}\right)$ из (15.1) с помошью леммы 8.2 при $\tau=0$ и с учетом выбора $C_{0}$, а также того, что $|x| \leqslant R_{s, \nu}$. Тогда

$$
\begin{aligned}
& \frac{1}{m_{0} !} 2^{-(S-s) m_{0}}\left|\Delta^{\left(m_{0}\right)}\left(2^{S-s} x, l_{0}, H\right)\right| q\left(\bar{l}^{\prime}\right) \\
& \quad \leqslant \delta\left(l_{0}, m_{0}, 0\right)\left(e\left(1+2^{S-s} \frac{|x|+1}{H}\right)\right)^{l_{0}} e^{C_{0}\left(L_{0}-l_{0}\right)} \\
& \quad \leqslant \delta\left(l_{0}, m_{0}, 0\right) e^{C_{0} L_{0}} \leqslant \delta\left(l_{0}, m_{0}, 0\right) e^{G}
\end{aligned}
$$

где

$$
\sum_{l_{0}=0}^{L_{0}} \delta\left(l_{0}, m_{0}, 0\right) \leqslant 1.6 e^{H / e} \leqslant 1.6 e^{0.4 \varepsilon_{1} G} .
$$

Осталось оценить $J$. С учетом (10.4) имеем

$$
\begin{aligned}
J & =\left(L_{0}+1\right)\left(\left[\left(\frac{1}{2} n \eta\right)^{-\varkappa} \frac{N}{\Omega} L^{n}\right]+1\right) \\
& \leqslant 2\left(1+\varepsilon_{1}\right) W_{0} \xi \omega\left(\frac{1}{2} n \eta\right)^{-\varkappa} \frac{N}{\Omega}\left(\frac{C_{0} \xi \omega}{c_{1}}\right)^{n} \\
& =\left(2\left(1+\varepsilon_{1}\right)\left(\frac{1}{2} n \eta\right)^{-\varkappa} \frac{\xi}{c_{1}} \frac{\left(20 n \xi / \xi_{0} e\right)^{n}}{\left(2 c_{1} W_{0}\right)^{n-1}} \gamma D\right)\left(\frac{e C_{0} W_{0} \omega \xi_{0}}{20 n}\right)^{n} \\
& \leqslant e^{(n-0.6) C_{0}} e^{0.1 \varepsilon_{1} G} \leqslant e^{(n-0.5) \varepsilon_{1} G} .
\end{aligned}
$$


Показатель $(n-0.6) C_{0}$ получен с учетом того, что $\gamma D$ входит в скобку в первой степени, а для зависимости от $n$ неравенство проверяется численно с учетом $2 c_{1} W_{0}>1 / \varepsilon_{2}$. В итоге получаем

$$
J \leqslant e^{(n-0.5) \varepsilon_{1} G} .
$$

Объединяя полученные оценки и учитывая множитель $\sqrt{J}$ для евклидовой нормы, получаем (15.5).

Лемма 15.2. Существует ненулевое решение $\mathbf{p} \in \mathbb{K}^{J}$ системы уравнений (9.9) для $s=0$, удовлетворяющее условию

$$
\|\mathbf{p}\|_{\mathscr{Q}} \leqslant e^{n \varepsilon_{1} G} .
$$

ДокАЗАТЕЛЬСТво. Оценим сначала $I$ и $J$. Мы имеем

$$
\begin{aligned}
I & =\left|\mathscr{X}_{0}\right| \Delta\left(M_{0}+1, n\right) \leqslant\left(2 X_{0}+1\right) \frac{1}{n !}\left(C_{0}\left(1+n \varepsilon_{1}\right) \xi \omega\right)^{n} \\
& \leqslant 4 c_{1} D W_{0}\left(1+\frac{1}{4} \varepsilon_{2}\right) \frac{1}{n !}\left(C_{0} \xi\left(1+n \varepsilon_{1}\right) \omega\right)^{n}
\end{aligned}
$$

а также

$$
\begin{aligned}
J & =\left(L_{0}+1\right)\left|\mathscr{L}_{0}\right|>W_{0} \xi \omega\left(\frac{1}{2} n \eta\right)^{-\varkappa} \frac{N}{\Omega}\left(\frac{C_{0} \xi \omega}{c_{1}}\right)^{n} \\
& =W_{0} \gamma D \xi\left(\frac{1}{2} n \eta\right)^{-\varkappa}\left(\frac{C_{0} \xi \omega}{c_{1}}\right)^{n} .
\end{aligned}
$$

Теперь оценим произведение в (13.1). Отметим, что оценка (15.4) для нормы $\left\|\mathbf{a}_{0}\left(x, \widetilde{m}^{\prime}\right)\right\|_{\mathscr{Q}^{\prime}}$ превосходит $q$ из $(15.2)$, так что

$$
\begin{aligned}
\Pi & =\prod_{i=1}^{I} \max \left\{q,\left\|\mathbf{a}_{i}\right\|_{\mathcal{Q}^{\prime}}\right\}=\prod_{x=-X_{0}}^{X_{0}} \prod_{\left|\tilde{m}^{\prime}\right| \leqslant M_{0}}\left\|\mathbf{a}_{0}\left(x, \tilde{m}^{\prime}\right)\right\|_{\mathscr{Q}^{\prime}} \\
& \leqslant \exp \left\{I n L \frac{X_{0}\left(X_{0}+1\right)}{\left(2 X_{0}+1\right) D}+\left(2+n \varepsilon_{1}\right) G I\right\} \\
& \leqslant \exp \left(I G\left(n+\frac{3}{2} n \varepsilon_{2}+2+n \varepsilon_{1}\right)\right) .
\end{aligned}
$$

Покажем, что $q^{J} \geqslant$ П. Для этого рассмотрим отношение

$$
\frac{\ln \Pi}{J \ln q} \leqslant \frac{I G\left(n+2 n \varepsilon_{2}+2+n \varepsilon_{1}\right)}{J C_{0} L_{0} / 2},
$$

в котором подстановка вместо $I, J$ их оценок из $(15.8),(15.9)$, с учетом обозначений из $\S 12$ после сокрашений одинаковых членов, дает

$$
\frac{\frac{8}{n !}\left(1+2 \varepsilon_{2}\right)\left(n+2 n \varepsilon_{2}+2+n \varepsilon_{1}\right)\left(\frac{1}{2} n \eta\right)^{\varkappa}\left(\left(1+n \varepsilon_{1}\right) c_{1}\right)^{n+1}}{\xi \gamma} .
$$


Согласно выбору параметра $\xi$ и неравенству (12.1) последняя величина не превосходит единицы. Отсюда следует, что выражение в скобках в (13.1) не больше 1. Таким образом, выполнение условий данного параграфа обеспечено в основном за счет выбора параметра $\xi$.

Для оценки сверху числа $J$ в (13.1) используем неравенство (15.7), а для оценки $|\Delta(\mathbb{K})|$ - лемму 14.2. Коэффициент при $\varepsilon_{1} G$ в показателе неравенства леммы равен $(n-0.5) / 2+0.3<n$. Лемма 15.2 доказана.

Наконец, обозначим вектор из пространства мультистепеней, соответствуюший какому-либо ненулевому значению $p\left(\bar{l}^{\prime}\right)$, через $\bar{v}_{0}$.

\section{§16. Интерполяционная формула}

В данном параграфе приводится количественный вариант интерполяционной формулы, основанной на теореме Коши о вычетах. Подобные леммы являются стандартным элементом техники арифметико-аналитического продолжения. Формулировка леммы взята из [1] с тем упрощением, что рассматриваются узлы только одинаковой кратности.

Пусть дано конечное множество $\mathscr{X} \subseteq \mathbb{C}$ (узлов интерполяции) и целое неотрицательное $T$ ( $T+1$ есть кратность узлов). Положим

$$
Q(z)=\prod_{x \in \mathscr{X}}(z-x)^{T+1} .
$$

Если $f(z)$ - функция, аналитическая в достаточно большом круге, то

$$
\begin{aligned}
f(z) & =f_{1}(z)+f_{2}(z) \\
f_{1}(z) & =\frac{1}{2 \pi i} \int_{\Gamma(0, R)} \frac{Q(z)}{Q(\zeta)} \cdot \frac{f(\zeta) d \zeta}{\zeta-z} \\
f_{2}(z) & =\sum_{x \in \mathscr{X}} \sum_{\tau=0}^{T} \frac{f^{(\tau)}(x)}{2 \pi i \tau !} \int_{\Gamma(x, r)} \frac{Q(z)}{Q(\zeta)} \cdot \frac{(\zeta-x)^{\tau} d \zeta}{\zeta-z} .
\end{aligned}
$$

Здесь окружность $\Gamma(x, r)$ с центром в $x$ радиуса $r$ обходится по часовой стрелке и не содержит внутри точку $z$ и остальные точки из $\mathscr{X} ; \Gamma(0, R)$ обходится против часовой стрелки и охватывает $z$ и все точки из $\mathscr{X}$. Будем называть выражение $Q(z) / Q(\zeta)$ интерполяционной частью.

ЛЕмма 16.1 [1, лемма 9.1]. Пусть даны $T \geqslant 0, \mathscr{X} \subseteq \mathbb{R}, z \in \mathbb{R} \backslash \mathscr{X}$. Точки $\mathscr{X}$ образуют арифметическую прогрессию с разностью $\delta>0$ и расположень симметрично относительно нуля. Положим

$$
z_{0}=\max \left\{|z|, \frac{1}{2} \delta|\mathscr{X}|\right\}, \quad r=\frac{1}{2} \min \{\delta, \min \{|z-x|: x \in \mathscr{X}\}\} .
$$

Тогда для $\zeta \in \mathbb{C} c|\zeta|>z_{0}$ выполняется

$$
\left|\frac{Q(z)}{Q(\zeta)}\right| \leqslant\left(\frac{z_{0}}{|\zeta|}\right)^{|\mathscr{X}|(T+1)},
$$

а для $\zeta \in \Gamma(x, r), \quad x \in \mathscr{X}$, выполняется

$$
\left|\frac{Q(z)}{Q(\zeta)}\right| \leqslant\left(\frac{2 e z_{0}}{\delta|\mathscr{X}|}\right)^{|\mathscr{X}|(T+1)} .
$$




\section{§17. Экстраполяция нулей}

Целью этого параграфа является доказательство индукционного шага по $\nu$ при фиксированном значении $s$ для уравнений (11.6), которые мы преобразуем к виду, аналогичному уравнениям (9.9):

$$
\begin{aligned}
\Phi_{s}\left(x, \widetilde{m}^{\prime}\right) & =0, \quad x \in \mathscr{X}_{s, \nu}, \quad\left\|\widetilde{m}^{\prime}\right\| \leqslant M_{s, \nu} \\
\nu & =0, \ldots, n, \quad s=0,1, \ldots, S
\end{aligned}
$$

где $\Phi_{s}\left(x, \widetilde{m}^{\prime}\right)$ определены в (9.8). Итак, мы считаем выполненными условия (17.1) при каких-либо $s$ и $\nu(0 \leqslant \nu<n)$ и хотим доказать, что они вьполнены и для $\nu+1$.

Для доказательства мы воспользуемся формулой произведения: если мы получим, что

$$
\prod_{\sigma=1}^{\infty}\left|\Phi_{s}\left(z, \tilde{m}^{\prime}\right)\right|_{\sigma}<1, \quad z \in \mathscr{X}_{s, \nu+1}
$$

то должно быть $\Phi_{s}\left(z, \widetilde{m}^{\prime}\right)=0$. Отметим, что при $z \in \mathbb{Z}$ выполняется

$$
\Phi_{s}\left(z, \widetilde{m}^{\prime}\right) \in \mathbb{K}
$$

При $\sigma>\varkappa$ мы будем оценивать $\left|\Phi_{s}\left(z, \widetilde{m}^{\prime}\right)\right|_{\sigma}$ тривиально с помошью (13.3), (15.5) и леммы 15.2. При $\sigma=1$ мы с помошью замены (9.4) перейдем к функциям $\Psi_{s}\left(\zeta, \widetilde{m}^{\prime}\right)$ из $(9.10)$ и будем оценивать их, пользуясь интерполяционной техникой $\S 16$ в предположении о малости линейной формы, взяв в качестве множества узлов интерполяции $\mathscr{X}=\mathscr{X}_{s, \nu}$ с кратностями $T=T_{s}$. В комплексном случае оценка при $\sigma=2$ будет такая же, как и при $\sigma=1$. В качестве функции $f(\zeta)$ из (16.1) возьмем

$$
f(\zeta)=\Psi_{s}\left(\zeta, \widetilde{m}^{\prime}\right) \bar{\alpha}^{\bar{v}_{s} z} \prod_{\sigma>\varkappa}\left|\Phi_{s}\left(z, \widetilde{m}^{\prime}\right)\right|_{\sigma}^{1 / \varkappa}
$$

При замене (9.4) и переходе от функций $\Phi$ к $\Psi$ возникает погрешность

$$
\begin{aligned}
\left|\bar{\alpha}^{\bar{l} \zeta}-\tilde{\alpha}^{\tilde{\chi}(\bar{l}) \zeta / b_{n}}\right| & =\left|e^{-\Lambda \zeta l_{n} / b_{n}}-1\right| \cdot\left|\bar{\alpha}^{\bar{l} \zeta}\right| \\
& \leqslant\left|\bar{\alpha}^{\bar{l} \zeta}\right| \cdot 1.1 \cdot\left|\Lambda \zeta l_{n}\right| \leqslant\left|\bar{\alpha}^{\bar{l} \zeta}\right| \cdot e^{1.1 \varepsilon_{1} G} \cdot|\Lambda|
\end{aligned}
$$

содержащая малый множитель $\Lambda$. Здесь мы использовали оценку

$$
1.1\left|\zeta l_{n}\right| \leqslant R_{s, \nu} 2^{-s} L / A_{n}=\eta\left|\mathscr{X}_{s, \nu}\right|\left(T_{s}+1\right) / A_{n},
$$

из которой с учетом (5.3), (11.5) получаем $1.1\left|\zeta l_{n}\right|<\exp \left(1.1 \varepsilon_{1} G\right)$. В итоге имеем

$$
\prod_{\sigma=1}^{\infty}\left|\Phi_{s}\left(z, \widetilde{m}^{\prime}\right)\right|_{\sigma}^{1 / \varkappa}=f(z)+\Delta_{0},
$$

где $\Delta_{0}$ - погрешность, возникаюшая при замене (9.4). 
Далее, применим к $f(z)$ интерполяционную формулу (16.1) и в выражениях для $f_{1}(z), f_{2}(z)$ сделаем обратную замену (9.4). В результате получим

$$
\prod_{\sigma=1}^{\infty}\left|\Phi_{s}\left(z, \widetilde{m}^{\prime}\right)\right|_{\sigma}^{1 / \varkappa}=J_{1}+\Delta_{1}+J_{2}+\Delta_{2}+\Delta_{0},
$$

где $J_{1}, J_{2}$ - соответствуюшие выражения в (16.1), а $\Delta_{1}, \Delta_{2}$ - погрешности, получающиеся в результате обратной замены (9.4).

В формуле (16.1) для $f_{2}(z)$ присутствуют производные $f^{(\tau)}$. Для них с учетом (9.10) имеем

$$
\begin{aligned}
& \frac{1}{\tau !} \Psi_{s}^{(\tau)}\left(x, \widetilde{m}^{\prime}\right)=\sum_{\left\|\tilde{\tau}^{\prime}\right\|=\tau} \sum^{(s)} \sum_{l_{0}=0}^{L_{0}} p\left(\bar{l}^{\prime}\right) \tilde{\alpha}^{\tilde{\chi}(\bar{l}) x / b_{n}} \bar{\alpha}^{-\bar{v}_{s} x} \\
& \times \frac{1}{m_{0} ! \tau_{0} !} 2^{-(S-s) m_{0}} \Delta^{\left(m_{0}+\tau_{0}\right)}\left(2^{S-s} z, l_{0}, H\right) \\
& \times d_{0}\left(m_{0}\right) \Delta\left(\tilde{\chi}\left(\bar{l}-\bar{w}_{s}\right), \tilde{m}\right)\left(\tilde{\chi}(\bar{l}) / b_{n}\right)^{\tilde{\tau}}(\ln \tilde{\alpha})^{\tilde{\tau}}(\tilde{\tau} !)^{-\tilde{1}}
\end{aligned}
$$

Отсюда и из леммы 3.1 видно, что $\Psi_{s}^{(\tau)}\left(x, \widetilde{m}^{\prime}\right)$ линейно выражаются через $\Psi_{s}\left(x, \tilde{t}^{\prime}\right),\left\|\tilde{t}^{\prime}\right\| \leqslant\left\|\tilde{m}^{\prime}\right\|+\tau$. То же самое будет и при обратном переходе (9.4) только для функций $\Phi$ вместо $\Psi$. Поэтому по предположению индукции (17.1) в (17.5) будет $J_{2}=0$.

Появление новых членов при дифференцировании полиномиальной части было заранее предусмотрено леммой 8.2 (в $\S 15$ мы пользовались только случаем $\tau=0$, но при выборе параметра $W_{0}$ учли и возможность роста $\left.\tau\right)$. При дифференшировании показательной части появилось выражение последней строки формулы (17.6). Оно предусмотрено леммой 8.3 , а (15.4) показывает, что параметр $W_{0}$ учитывает все новые компоненты. Поэтому в (17.6) из (8.5), (15.4) имеем

$$
d_{0}\left(m_{0}\right) \sum_{\|\tilde{\tau}\| \leqslant M_{0}-m_{0}}\left|\Delta\left(\tilde{\chi}\left(\bar{l}-\bar{w}_{s}\right), \tilde{m}\right)\left(\tilde{\chi}(\bar{l}) / b_{n}\right)^{\tilde{\tau}}(\ln \tilde{\alpha})^{\tilde{\tau}}(\tilde{\tau} !)^{-\tilde{1}}\right| \leqslant 0.01 e^{G} .
$$

Поскольку в лемме 15.2 для $x$ были предусмотрены границы, охватываюшие все встречаемые далее значения $z, \zeta$, то оценка полиномиальной части будет такой же, как в $\S 15$.

В показательной части (15.6) произошли некоторые изменения. Компонента $|\bar{\alpha}|_{\sigma}^{\bar{v}_{s} z}$ при умножении по всем $\sigma$ за счет формулы произведения исчезнет. Но сейчас мы рассматриваем не абсолютные высоты (извлечение корня степени $D$ ), а извлекаем корень степени $\varkappa$. Поскольку тело $\mathscr{W}$ расположено симметрично относительно нуля, то все локальные высоты показательной части неотрицательны, и отсутствие сомножителя при $\sigma=1, \varkappa$ только упрощает ситуащию и позволяет воспользоваться прежней оценкой показательной части:

$$
\frac{1}{\varkappa} 2^{-s} \operatorname{Ln}|z| \leqslant \frac{1}{\varkappa} 2^{-s} \operatorname{Ln} 2^{\nu+1} X_{s} .
$$


Кроме того, в $J_{1}$ у нас появился показательньй сомножитель, содержащий $\zeta$, который из-за условия (10.3) оценивается как $\exp \left(\eta^{-1} 2^{-s} L|\zeta|\right)$.

Для оценки интерполяционной части $|Q(z) / Q(\zeta)|$ в $J_{1}$ мы используем (16.2), при этом для оценки $\operatorname{deg} Q=\left|\mathscr{X}_{s, \nu}\right|\left(T_{s}+1\right)$ имеется двустороннее неравенство (11.5).

Отметим, что для $z \in \mathscr{X}_{s, \nu+1}$ в лемме 16.1 выполняется неравенство $z_{0} \leqslant$ $\left|\mathscr{X}_{s, \nu}\right|$. Тогда, собрав оценки для компонент $J_{1}$ из лемм $15.1,15.2$ с учетом (13.2), (11.5), а также положив $|\zeta|=R=R_{s, \nu}$, имеем

$$
\begin{aligned}
\ln \left(8\left|J_{1}\right|\right) \leqslant & \frac{1}{\varkappa}\left(2^{\nu+1} X_{s}\right)\left(2^{-s} L\right) n+\frac{D}{\varkappa}\left(2+n \varepsilon_{1}\right) G \\
& +\eta^{-1} 2^{-s} L R_{s, \nu}+\left|\mathscr{X}_{s, \nu}\right|\left(T_{s}+1\right) \ln \left(\frac{\left|\mathscr{X}_{s, \nu}\right|}{R_{s, \nu}}\right) \\
\leqslant & \frac{1}{\varkappa}\left|\mathscr{X}_{s, \nu}\right|\left(T_{s}+1\right) n+\frac{D}{\varkappa} 2.1 G \\
& -\left|\mathscr{X}_{s, \nu}\right|\left(T_{s}+1\right) \ln \left(e^{(n+0.53) / \varkappa} \frac{\left(T_{s}+1\right)}{2^{-s} L}\right) \\
\leqslant & \frac{1}{\varkappa}\left|\mathscr{X}_{s, \nu}\right|\left(T_{s}+1\right)\left(n+\frac{1}{4} \cdot 2.1-(n+0.53)\right)<0,
\end{aligned}
$$

т.е. $\left|J_{1}\right|<1 / 8$. А из (17.4), (12.1) находим $\left|\Delta_{1}\right| \leqslant 2\left|J_{1}\right|<1 / 4$. Выполнение этих неравенств в основном обеспечено за счет выбора параметра $\eta$ из (10.3).

Малость $\Delta_{0}, \Delta_{2}(<1 / 4)$ будет обеспечена за счет гипотезы $(12.2)$ о малости $|\Lambda|$ и проверена в следуюшем параграфе. Здесь $J_{2}=0$. Тогда из формулы (17.5) получаем неравенство (17.2), и тем самым доказываем шаг индукции.

\section{§18. Деление аргумента}

В данном параграфе будет доказан индукционный шаг по $s$, т.е. из равенств (11.6) (эквивалентно (17.1)) при $(s, n)$ будут получены такие же равенства при $(s+1,0)$.

Сначала мы получаем равенства

$$
F_{s}\left(\frac{x}{2}, \widetilde{m}^{\prime}\right)=0, \quad\left\|\widetilde{m}^{\prime}\right\| \leqslant M_{s+1}, \quad x \in \mathscr{X}_{s+1,0}
$$

а затем с помошью теории Куммера - условия (17.1).

Мы докажем (18.1) в конще параграфа, а пока посмотрим, что из этих равенств следует. Обратимся к способу построения множеств $\mathscr{L}_{s}$. В расширенном логарифмическом пространстве числам $\alpha_{1}, \ldots, \alpha_{n}$ соответствуют векторы $\mathbf{a}_{1}, \ldots, \mathbf{a}_{n}$ (согласно условию теоремы 2.1 линейно независимые над $\mathbb{Z}$ ). По ним построена решетка $\mathrm{M}_{n}$ с базисом $\mathbf{e}_{1}, \ldots, \mathbf{e}_{n}$. Этим векторам соответствуют в поле $\mathbb{K}$ числа $\vartheta_{1}, \ldots, \vartheta_{n}$, обладающие важным алгебраическим свойством.

Лемма 18.1. Пусть $\mathbb{K}$ содержит корень степени $p$ из 1 ( $p$ - некоторое простое число). Тогда вышеприведенные $\vartheta_{1}, \ldots, \vartheta_{n}$ удовлетворяют условию

$$
\left[\mathbb{K}\left(\vartheta_{1}^{1 / p}, \ldots, \vartheta_{n}^{1 / p}\right): \mathbb{K}\right]=p^{n}
$$


ДокАЗАТЕЛЬСтво. Лемма практически доказана в работах [15] (см. также [4]), но поскольку явно не сформулирована, мы приведем ее доказательство.

Так как поле $\mathbb{K}$ содержит корень степени $p$ из 1, то для доказательства неважно, какое значение $\vartheta_{i}^{1 / p}$ выбирается. Мы будем считать его согласованным с выбором $\ln \vartheta_{i}$.

Доказывать будем индукцией по $j$, рассматривая поля

$$
\mathbb{K}_{0}=\mathbb{K}=\mathbb{Q}\left(\vartheta_{1}, \ldots, \vartheta_{n}\right), \quad \mathbb{K}_{j}=\mathbb{K}\left(\vartheta_{1}^{1 / p}, \ldots, \vartheta_{j}^{1 / p}\right), \quad 1 \leqslant j \leqslant n .
$$

Надо доказать, что $\left[\mathbb{K}_{j+1}: \mathbb{K}_{j}\right]=p$. Если при каком-либо $j$ это не так, то при некоторых $\alpha \in \mathbb{K}$ и $r_{i} \in\{0,1, \ldots, p-1\}, 1 \leqslant i \leqslant j$, выполняется

$$
\vartheta_{j+1}=\vartheta_{1}^{r_{1}} \ldots \vartheta_{j}^{r_{j}} \alpha^{p}
$$

(см. [15, лемма 3]). Но тогда в расширенном логарифмическом пространстве вектор, соответствуюший числу $\alpha$ с естественным выбором ветви логарифма, линейно зависим над $\mathbb{Z} \mathbf{c} \mathbf{e}_{1}, \ldots, \mathbf{e}_{n}$, но целочисленно через них не выражается, что противоречит условию о том, что векторы $\mathbf{e}_{j}$ образуют базис решетки $\mathrm{M}_{n}$. Это и доказывает лемму.

Мы будем использовать лемму 18.1 при $p=2$, что согласуется с (1.2) и исключает вопросы о корне из 1 (равном здесь -1 ).

Вернемся к доказательству теоремы. Координатам в $\mathrm{M}_{n}$ соответствуют мультистепени при соответствуюших наборах чисел, при этом согласно (10.1)

$$
\bar{\alpha}^{\bar{\lambda}}=\bar{\vartheta}^{\bar{\mu}}, \quad \bar{\lambda} \in \mathscr{M}_{n}, \quad \bar{\mu} \in \mathbb{Z}^{n}, \quad \bar{\lambda}=\bar{\mu} \mathscr{A}^{-1},
$$

где $\mathscr{A}$ - матрица перехода от базиса $\left\{\mathbf{a}_{i}\right\}$ к базису $\left\{\mathbf{e}_{j}\right\}$.

Теперь положим

$$
x=2 y+1, \quad \bar{\mu}=2 \bar{\tau}+\bar{\delta}, \quad \bar{\delta} \in\{0,1\}^{n}, \quad \bar{\tau} \in \mathbb{Z}^{n} .
$$

Тогда из (18.1) с учетом замены (18.2) и отбрасывания общего множителя $\bar{\alpha} \bar{v} x / 2$ имеем

$$
\begin{aligned}
0 & =F_{s}\left(\frac{x}{2}, \widetilde{m}^{\prime}\right)=\sum_{\bar{\mu}}^{(s)} P_{s}^{\left(m_{0}\right)}\left(\frac{x}{2}, \bar{l}\right) \tilde{\chi}^{\widetilde{m}}(\bar{l}) \bar{\vartheta}^{\bar{\mu} x / 2} \\
& =\sum_{\bar{\delta}}\left(\sum_{\bar{\tau}} P_{s}^{\left(m_{0}\right)}\left(\frac{x}{2}, \bar{l}\right) \tilde{\chi}^{\widetilde{m}}(\bar{l}) \bar{\vartheta}^{\bar{\tau} x+\bar{\delta} y}\right) \bar{\vartheta}^{\bar{\delta} / 2}
\end{aligned}
$$

В этот момент мы используем условие Куммера, согласно которому числа $\bar{\vartheta} \bar{\delta} / 2$, $\bar{\delta} \in\{0,1\}^{n}$, линейно независимы над $\mathbb{K}$. Множители в (18.3), стоящие перед $\bar{\vartheta}^{\bar{\delta} / 2}$, из поля $\mathbb{K}$, значит, все они исчезают, и мы получаем, что в (18.3) слагаемые при каждом значении $\bar{\delta}$ равны нулю, или после обратного перехода с помошю (18.2) к вектору $\bar{\alpha}$, что

$$
\begin{aligned}
0 & =\sum_{\bar{\tau}}^{(s+1)} P_{s}^{\left(m_{0}\right)}\left(\frac{x}{2}, \bar{l}\right) \tilde{\chi}^{\widetilde{m}}(\bar{l}) \bar{\vartheta}^{\bar{\mu} x / 2} \\
& =\sum_{\bar{\tau}}^{(s+1)} P_{s}^{\left(m_{0}\right)}\left(\frac{x}{2}, \bar{l}\right) \tilde{\chi}^{\widetilde{m}}(\bar{l}) \bar{\alpha}^{\bar{\lambda} x / 2}
\end{aligned}
$$


Мы будем работать только с $\bar{\delta}$, соответствуюшим изначально выбранному значению $\bar{l}$, для которого имеется ненулевое $p\left(l_{0}, \bar{l}\right)$. Переобозначив $\bar{\lambda} / 2$ и учитывая, что $\bar{\tau}$ выражается через $\bar{\lambda}$, мы можем считать, что суммирование в (18.4) проводится по $\bar{\lambda} \in \mathscr{L}_{s+1}$. Это согласуется с требованиями к $\mathscr{L}_{s+1}$ из $\S 9,10$, поскольку, очевидно, $\mathscr{L}_{s+1} \subseteq \mathscr{L}_{s} / 2$ и

$$
\frac{1}{2} \bar{\lambda}=\frac{1}{2} \bar{\mu} \mathscr{A}^{-1}=\bar{\tau} \mathscr{A}^{-1}+\frac{1}{2} \bar{\delta} \mathscr{A}^{-1} \in \mathscr{M}_{n}+\frac{1}{2} \bar{\delta} \mathscr{A}^{-1}
$$

Мы не трогаем индексы $\bar{l}$ в полиномиальной и дифференциальной части, подразумевая, что они выражаются через $\bar{\lambda}$ и $\bar{\delta}$. Теперь снова домножив выражение в (18.4) на $\bar{\alpha} \bar{v} x / 2$ и обозначив его через $F_{s+1}$, мы получаем выполнение условия (11.6) при $s+1$ и $\nu=0$.

Перейдем теперь к доказательству (18.1), где функции $F$ могут быть заменены на $\Phi$. В отличие от $\S 17$ здесь нам приходится работать в поле $\mathbb{K}_{n}$ степени $2^{n} D$. Пронумеруем его нормирования индексами $\sigma$ и будем рассматривать функцию $f(\zeta)$ из (17.3) как определенную над $\mathbb{K}_{n}$. Параметром $z$ в ней будет

$$
z=\frac{1}{2} x, \quad x \in \mathscr{X}_{s+1}, \quad|z| \leqslant X_{s+1} .
$$

Аналогичным образом определяются в (17.5) величины $\Delta_{0}, J_{1}, \Delta_{1}, J_{2}, \Delta_{2} \mathrm{c} \nu=n$. При этом также выполнено $J_{2}=0$. В лемме 16.1 полагаем $T=T_{s}, \mathscr{X}=\mathscr{X}_{s, n}$, $\delta=1$ (разность прогрессии), $r=1 / 4, z_{0}=\left|\mathscr{X}_{s, n}\right| / 2$, а радиус $R=R_{s, n}$ определен в $\S 12$.

Оценка $J_{1}$ получается, как в $(17.7)$, только с учетом новой границы для $z(|z|=$ $|x| / 2 \leqslant X_{s+1}$, см. (11.4)) и степени поля:

$$
\begin{aligned}
\ln \left(8\left|J_{1}\right|\right) \leqslant & \frac{2^{n}}{\varkappa} 2^{-s} \operatorname{Ln} X_{s+1}+\frac{2^{n} D}{\varkappa}\left(2+n \varepsilon_{1}\right) G \\
& +\eta^{-1} 2^{-s} L R_{s, n}+\left|\mathscr{X}_{s, n}\right|\left(T_{s}+1\right) \ln \left(\left|\mathscr{X}_{s, n}\right| / 2 R_{s, n}\right) \\
\leqslant & \frac{2^{n}}{\varkappa} 2^{-s} \operatorname{Ln} X_{s+1}+\frac{2^{n} D}{\varkappa}\left(2+n \varepsilon_{1}\right) G \\
& -\left|\mathscr{X}_{s, n}\right|\left(T_{s}+1\right) \ln \left(e^{(n+0.53) / \varkappa} \frac{\left(T_{s}+1\right)}{2^{1-s} L}\right) \\
\leqslant & \frac{D}{\varkappa} 2^{n+2} G\left(n\left(1+\varepsilon_{2}\right)+\frac{1}{4}\left(2+n \varepsilon_{1}\right)-(n+0.53)\right)<0,
\end{aligned}
$$

откуда $\left|J_{1}\right|<1 / 8$ и $\left|\Delta_{1}\right|<1 / 4$ (см. $\left.\S 17\right)$. Это также в основном обеспечено за счет выбора параметра $\eta$.

Осталось убедиться, что предположение (12.2) обеспечивает $\left|\Delta_{0}\right|,\left|\Delta_{2}\right|<1 / 4$. Тогда (17.2) будет выполнено и шаг индукции доказан. Мы будем брать худший случай на всех шагах индукции, получающийся при переходе от $s$ к $s+1$.

Оценка $\Delta_{2}$ проводится аналогично оценке $J_{1}$. Отличия состоят в следуюшем. При оценке $J_{1}$ нам надо было мажорировать в формуле для $f_{1}(z)$ из (16.1) величину $|f(\zeta)|,|\zeta| \leqslant R_{s, \nu}$. При оценке $\Delta_{2}$ нам надо мажорировать $\left|f^{(\tau)}(x)\right|,|x| \leqslant 2^{n} X_{s}$. Из (17.6) видно, что оценка $\left|f^{(\tau)}(x)\right| / \tau$ ! такая же, как оценка $|f(\zeta)|$ (это (17.7) 
при $\nu<n$ и (18.5) при $\nu=n)$, только $R_{s, \nu}$ надо заменить на $2^{n} X_{s}$. Далее, при оценке интерполяционной части вместо неравенства (16.2) следует использовать неравенство (16.3), с учетом формулы для $z_{0}$ имеющего вид

$$
\begin{aligned}
& \ln |Q(z) / Q(\zeta)| \leqslant\left|\mathscr{X}_{s, \nu}\right|\left(T_{s}+1\right) \ln (2 e), \quad \nu<n, \\
& \ln |Q(z) / Q(\zeta)| \leqslant\left|\mathscr{X}_{s, \nu}\right|\left(T_{s}+1\right) \ln (e), \quad \nu=n .
\end{aligned}
$$

Так что во второй строке неравенств (17.7), (18.5) второе слагаемое, соответствующее оценке (16.2), следует заменить на вьшеуказанную оценку.

Кроме того, надо добавить в оценку множитель из (17.4):

$$
|\Lambda| R_{s, n} 2^{-s} L / A_{n}<|\Lambda| e^{1.1 \varepsilon_{1} G} .
$$

И, наконец, надо оценить еще сумму в $f_{2}$ из (16.1). Ранее этого не требовалось, так как было $J_{2}=0$. Поскольку во всех случаях имеем $r \leqslant 1 / 2$, то сумма по $\tau$ в $(16.1)$ с учетом наличия в интеграле члена $r^{\tau}$ оценивается числом 2 . Так что суммирование в $f_{2}$ даст $4|\mathscr{X}| \leqslant e^{1.1 \varepsilon_{1} G}$. В итоге, взяв худший случай $\nu=n$, получаем

$$
\begin{aligned}
\ln \left(8\left|\Delta_{2}\right|\right) \leqslant & \frac{1}{\varkappa} 2^{-s} L n 2^{n} X_{s+1}+\frac{D}{\varkappa} 2^{n}\left(2+3 n \varepsilon_{1}\right) G \\
& +\eta^{-1} 2^{-s} L 2^{n} X_{s}+\left|\mathscr{X}_{s, n}\right|\left(T_{s}+1\right)+\ln |\Lambda| \\
\leqslant & \frac{D}{\varkappa} 2^{n+1} G\left(1+2 \varepsilon_{2}\right)\left(2 n+1+2 n \varepsilon_{1}+\frac{\varkappa}{\eta}+2 \varkappa\right)+\ln |\Lambda|,
\end{aligned}
$$

и с учетом $(11.5),(12.1)$ и предположения $(12.2)$ выполнено $\ln \left|8 \Delta_{2}\right|<0$. Аналогично, для $\Delta_{0}$ можно получить неравенство $\left|\Delta_{0}\right|<1 / 2$. Тогда с помошью $(17.5),(17.2)$ завершаются рассуждения, связанные с экстраполяцией нулей, и аналогично доказывается индукционный шаг.

\section{§19. Оценка кратностей нулей}

В данном параграфе доказывается оценка кратностей нулей для показательного квазимногочлена от многих переменных. Эта теорема представляет собой адаптированную версию результата Вюстхольца [19] специально для случая однородных линейных форм от логарифмов алгебраических чисел. В формулировке учтены нюансы, указанные в работах [4], [17], [20]. Переработке подверглась геометрическая часть рассуждений из работ [19], [4], что позволило улучшить значение константы $\mathscr{C}(\nu)$ (см. предложение 19.2). Для формулировки будем использовать буквенные обозначения, связь которых с параметрами из предыдущих параграфов будет установлена в $\S 20$. Кроме того, для формулировки и доказательства утверждений данного параграфа нам потребуются некоторые понятия об алгебраических группах (см. [19], [20]).

Алгебраические подгруппы в $\mathbb{G}_{a} \times \mathbb{G}_{m}^{n}$ имеют вид $\mathbb{H}=\mathbb{H}_{a} \times \mathbb{H}_{m}, \mathbb{H}_{a} \subseteq \mathbb{G}_{a}$, $\mathbb{H}_{m} \subseteq \mathbb{G}_{m}$, при этом либо $\mathbb{H}_{a}=\{0\}$, либо $\mathbb{H}_{a}=\mathbb{G}_{a}$.

Далее, при $\nu>0$ связная группа $\mathbb{I}_{m}$ ранга $\nu$ определяется соотношениями вида $\bar{Y}^{\bar{l}}=1$, где $\bar{l} \in \mathrm{M}$, а $\mathrm{M}=\left\langle\bar{v}_{1}, \ldots, \bar{v}_{\nu}\right\rangle_{\mathbb{Z}} \subseteq \mathbb{Z}^{n}$ - некоторая решетка ранга $\nu$. Тогда $r=\operatorname{rank} \mathbb{H}=\nu+\varepsilon$, где $\varepsilon$ равно 0 или 1 в зависимости от $\mathbb{H}_{a}$. Положим

$$
d\left(j_{1}, \ldots, j_{\nu}\right)=\left|\operatorname{det}\left(v_{i, j_{k}}\right)_{1 \leqslant i, k \leqslant \nu}\right|, \quad 1 \leqslant j_{1}<\cdots<j_{\nu} \leqslant n,
$$


и отметим, что эти выражения не зависят от выбора базиса М.

Наконец, с $\mathbb{H}$ связан многочлен Гильберта, для коэффициентов $\delta\left(\mathbb{H} ; i_{1}, \ldots, i_{r}\right)$ которого нам понадобится оценка

$$
\begin{aligned}
\delta\left(\mathbb{H} ; 0, i_{2}, \ldots, i_{r}\right) \geqslant d\left(i_{2}, \ldots, i_{r}\right), & r=\nu+1 \\
\delta\left(\mathbb{H} ; i_{1}, \ldots, i_{r}\right) \geqslant d\left(i_{1}, \ldots, i_{r}\right), \quad r & =\nu .
\end{aligned}
$$

Теперь пусть даны набор чисел $\bar{b}=\left(b_{1}, \ldots, b_{n}\right), b_{n} \neq 0$, и набор $\bar{\vartheta}=\left(\vartheta_{1}, \ldots, \vartheta_{n}\right)$ ненулевых алгебраических чисел. В кольце многочленов $\mathbb{C}\left[Y_{0}, \ldots, Y_{n}\right]$ рассматриваются операторы дифференцирования $\partial_{j}$, определенные в (3.1).

Пусть также даны целые числа:

$$
\begin{gathered}
L_{0}^{\prime}, \ldots, L_{n}^{\prime} \geqslant 0 \quad \text { (степени) } \\
X_{0}^{\prime} \geqslant \cdots \geqslant X_{n}^{\prime} \geqslant 0, \quad X_{0}^{\prime}+\cdots+X_{n}^{\prime} \leqslant X^{\prime} \quad \text { (нули) }, \\
M_{0}^{\prime} \geqslant \cdots \geqslant M_{n}^{\prime} \geqslant 0, \quad M_{0}^{\prime}+\cdots+M_{n}^{\prime} \leqslant M^{\prime} \quad \text { (кратности) },
\end{gathered}
$$

удовлетворяющие дополнительному условию: при всех $0 \leqslant \nu \leqslant n$ и при всех $\bar{m}^{\prime}=\left(m_{0}, \ldots, m_{n}\right) \in\{0,1\}^{n+1},\left\|\bar{m}^{\prime}\right\|=\nu+1$ ( $\|\cdot\|$-sum-норма $)$, выполняется неравенство

$$
\left(X_{\nu}^{\prime}+1\right)\left(\begin{array}{c}
M_{\nu}^{\prime}+\nu+1-\delta_{\nu, n} \\
\nu+1-\delta_{\nu, n}
\end{array}\right)>(\nu+1) ! L_{0}^{\prime m_{0}} \ldots L_{n}^{\prime m_{n}}
$$

где $\delta_{\nu, n}-$ символ Кронекера и полагается $0^{0}=1$.

Выделим также для рассматриваемой группы $\mathbb{H}$ случаи:

(i) Lie $\mathbb{H} \subseteq\left(\partial_{0}, \ldots, \partial_{n-1}\right)$; тогда положим $\sigma=r-1$, иначе $\sigma=r$;

(ii) для некоторого $x \in\left\{1, \ldots, X^{\prime}\right\}$ имеем $\left(x, \vartheta_{1}^{x}, \ldots, \vartheta_{n}^{x}\right) \in \mathbb{H}(\overline{\mathbb{Q}})$; тогда положим $E=1$, иначе $E=X_{r-1}^{\prime}+1$.

ПРЕДЛОЖЕНИЕ 19.1 [19, теорема 1.1]. Пусть в указанных обозначениях имеется многочлен $P \in \mathbb{C}\left[Y_{0}, \ldots, Y_{n}\right], \quad P \neq 0, \operatorname{deg}_{Y_{j}} P \leqslant L_{j}^{\prime}, \quad 0 \leqslant j \leqslant n$, удовлетворяющий условию

$$
\partial_{0}^{\mu_{0}} \ldots \partial_{n-1}^{\mu_{n-1}} P\left(x, \vartheta_{1}^{x}, \ldots, \vartheta_{n}^{x}\right)=0
$$

для $x=0, \ldots, X^{\prime}$ u всех $\tilde{\mu}^{\prime} \in \mathbb{N}_{0}^{n} c\left\|\tilde{\mu}^{\prime}\right\| \leqslant M^{\prime}$.

Тогда найдется связная алгебраическая группа $\mathbb{H} \subseteq \mathbb{G}_{a} \times \mathbb{G}_{m}^{n}$ ранга $r \leqslant n$, для которой при любых $0 \leqslant i_{1}<\cdots<i_{r} \leqslant n$ выполняется

$$
\begin{gathered}
\delta\left(\mathbb{H} ; i_{1}, \ldots, i_{r}\right) \leqslant \gamma_{r} L_{i_{1}}^{\prime} \ldots L_{i_{r}}^{\prime} \\
\gamma_{r}=r ! E^{-1}\left(\begin{array}{c}
M_{r-1}^{\prime}+\sigma \\
\sigma
\end{array}\right)^{-1} .
\end{gathered}
$$

Перейдем к формулировке той оценки кратностей нулей, на которую мы будем опираться для завершения доказательства основной теоремы 2.1. Предварительно введем несколько дополнительных ограничений, естественным образом получающихся из предыдущих параграфов. Все они сужают возможные случаи предложения 19.1. 
У нас имеется набор $\bar{A}=\left(A_{1}, \ldots, A_{n}\right)$ положительных чисел и фиксированы значения $\ln \vartheta_{1}, \ldots, \ln \vartheta_{n}$, при этом логарифмы линейно независимы над $\mathbb{Z}$ и выполняются неравенства

$$
\begin{gathered}
\left|b_{1} \ln \vartheta_{1}+\cdots+b_{n} \ln \vartheta_{n}\right|<\frac{2 \pi}{X^{\prime}+1}, \\
M_{0}^{\prime}+1>L_{j}^{\prime}, \quad 1 \leqslant j \leqslant n \\
n(n+1) L_{0}^{\prime}>M_{n}^{\prime}+n .
\end{gathered}
$$

ПРЕДЛОЖЕНИЕ 19.2. При выполнении условий (19.1), (19.2), (19.4)--(19.6) найдутся $\nu(1 \leqslant \nu<n)$ и набор независимых векторов $\bar{Z}_{i} \in \mathbb{Z}^{n}, \quad 1 \leqslant i \leqslant \nu$, такие, что $\bar{b} \in\left\langle\bar{Z}_{1}, \ldots, \bar{Z}_{\nu}\right\rangle_{\mathbb{Q}}$ и для чисел

$$
A_{i}^{\prime}=\left\|\bar{Z}_{i}\right\|_{A}=A_{1}\left|Z_{i, 1}\right|+\cdots+A_{n}\left|Z_{i, n}\right|, \quad 1 \leqslant i \leqslant \nu,
$$

выполняется хотя бы одно из двух неравенств:

$$
\begin{aligned}
& \left(X_{\nu-1}^{\prime}+1\right)\left(\begin{array}{c}
M_{\nu-1}^{\prime}+\nu-1 \\
\nu-1
\end{array}\right) A_{1}^{\prime} \ldots A_{\nu}^{\prime} \leqslant \mathscr{C}(\nu) \max \left\{\bar{A}^{\bar{m}} \bar{L}^{\prime \bar{m}}\right\} \\
& \left(X_{\nu}^{\prime}+1\right)\left(\begin{array}{c}
M_{\nu}^{\prime}+\nu \\
\nu
\end{array}\right) A_{1}^{\prime} \ldots A_{\nu}^{\prime} \leqslant \mathscr{C}(\nu)(\nu+1) L_{0}^{\prime} \max \left\{\bar{A}^{\bar{m}} \bar{L}^{\prime \bar{m}}\right\}
\end{aligned}
$$

где максимум берется по наборам $\bar{m} \in\{0,1\}^{n},\|\bar{m}\|=\nu, a$

$$
\mathscr{C}(\nu)=2^{\nu}\left(\frac{2 n}{\pi}\right)^{\nu / 2}\left(\frac{\nu}{2}\right) ! \nu !\left(\begin{array}{l}
n \\
\nu
\end{array}\right)^{1 / 2}
$$

ПримечАниЕ. В работе [4] было $\mathscr{C}(\nu)=2^{1-\nu}(\nu !)^{2}(n \nu)^{\nu}$, а также было требование примитивности набора векторов $\bar{Z}_{i}$.

ДокАЗАТЕЛЬСТво. Сначала, как и в [4], исключим некоторые возможности предложения 19.1. В случае (ii) мы имеем соотношения

$$
l_{1} \ln \vartheta_{1}+\cdots+l_{n} \ln \vartheta_{n}=\frac{2 \pi i}{x} l_{0}, \quad 1 \leqslant x \leqslant X^{\prime}, \quad l_{0} \in \mathbb{Z}, \quad \bar{l} \in \mathrm{M},
$$

где $\mathrm{M}$ - решетка ранга $\nu=r$, определяюшая группу $\mathbb{H}_{m}$. Линейная независимость чисел $\ln \vartheta_{j}$ дает, что должно быть $\nu=1$ (иначе $2 \pi i$ можно исключить) и при этом $l_{0} \neq 0$. Вектор $\bar{l}$ можно считать примитивньм. Если при этом имеем еще случай (i), то $\bar{b}=m_{0} \bar{l}, m_{0} \in \mathbb{Z}$. Но тогда это противоречит условию (19.4), поэтому случай (i) не выполняется, и мы имеем $\sigma=r=\nu=1, E=1, \gamma_{r}=\left(M_{0}^{\prime}+1\right)^{-1}$. Но тогда (19.3) противоречит условию (19.5), поскольку для какого-то из индексов мы имеем $\delta(\mathbb{H} ; i) \geqslant 1,1 \leqslant i \leqslant n$.

Таким образом, случай (ii) полностью исключен и $E=X_{r-1}^{\prime}+1$.

Если не выполняется случай (i), то $\sigma=r$ и рассмотрение $\delta\left(\mathbb{H} ; i_{1}, \ldots, i_{r}\right) \geqslant 1$ приводит (19.3) в противоречие с (19.1). 
В случае (i) мы имеем $\bar{b} \in\langle\mathrm{M}\rangle_{\mathbb{Q}}$, так что $\nu \geqslant 1$. Если предположить $\nu=n$, то ввиду $\nu \leqslant r \leqslant n$ имеем $r=n=\sigma$ и рассмотрение $\delta(\mathbb{H} ; 1 \ldots, n) \geqslant 1$ приводит (19.3) в противоречие с (19.1) при $\nu=n$ (с учетом (19.6) и монотонности $X_{j}^{\prime}, M_{j}^{\prime}$ ).

Таким образом, мы нашли нужную решетку $\mathrm{M} \subseteq \mathbb{Z}^{n}$ ранга $\nu(1 \leqslant \nu<n)$. Осталось доказать существование независимых векторов $\bar{Z}_{1}, \ldots, \bar{Z}_{\nu} \in \mathrm{M}$, удовлетворяющих условиям (19.7) или (19.8).

В пространстве $\mathscr{V}_{n}=\mathbb{R}^{n}$ у нас есть sum-норма $\|\cdot\|_{A}$. Ее единичный шар - это октаэдр $\mathscr{W}_{n}$, задаваемый в стандартных координатах $\left(x_{1}, \ldots, x_{n}\right)$ неравенством $\left|x_{1}\right| A_{1}+\cdots+\left|x_{n}\right| A_{n} \leqslant 1$.

Введем в $\mathscr{V}_{n}$ новые координаты $x_{j}^{\prime}=A_{j} x_{j}, 1 \leqslant j \leqslant n$, и будем считать их евклидовыми. Тогда в прежних координатах $\left\{x_{j}\right\}$ матрица соответствующей квадратичной формы будет иметь вид $Q=\operatorname{diag}\left\{A_{1}^{2}, \ldots, A_{n}^{2}\right\}$. Обозначим введенную метрику через $|\cdot|_{Q}$ :

$$
|\mathbf{v}|_{Q}^{2}=x_{1}^{\prime 2}+\cdots+x_{n}^{\prime 2}=\left(x_{1}, \ldots, x_{n}\right) Q\left(x_{1}, \ldots, x_{n}\right)^{T} .
$$

В новых координатах октаэдр $\mathscr{W}_{n}$ определяется условием $\left|x_{1}^{\prime}\right|+\cdots+\left|x_{n}^{\prime}\right| \leqslant 1$, т.е. является правильным. Легко видеть, что $Q$-евклидов шар $\mathscr{S}$, задаваемый условием

$$
|\mathbf{v}|_{Q}^{2}=x_{1}^{\prime 2}+\cdots+x_{n}^{\prime 2} \leqslant R^{2}
$$

при $R=1 / \sqrt{n}$ будет содержаться в $\mathscr{W}_{n}$.

Оценим $\nu$-мерный $Q$-евклидов объем решетки М. Выберем какой-либо базис $\bar{v}_{1}, \ldots, \bar{v}_{\nu}$ решетки. Пусть $V$-матрица строк координат базисных векторов: тогда, применяя формулу Бине-Коши, а также (19.3) с $\sigma=r-1$ (случай (i)), имеем

$$
\begin{aligned}
\operatorname{Vol}_{\nu}^{2} \mathrm{M} & =\operatorname{det}\left(V Q V^{T}\right)=\sum A_{j_{1}}^{2} \ldots A_{j_{\nu}}^{2} d^{2}\left(j_{1}, \ldots, j_{\nu}\right) \\
& \leqslant \sum\left(A_{j_{1}} \ldots A_{j_{\nu}} \gamma_{r} L_{0}^{\prime \varepsilon} L_{j_{1}}^{\prime} \ldots L_{j_{\nu}}^{\prime}\right)^{2} \leqslant\left(\begin{array}{c}
n \\
\nu
\end{array}\right)\left(\gamma_{r} L_{0}^{\prime \varepsilon} \max \left\{\bar{A}^{\bar{m}} \bar{L}^{\prime \bar{m}}\right\}\right)^{2} .
\end{aligned}
$$

Здесь сумма берется по наборам $1 \leqslant j_{1}<\cdots<j_{\nu} \leqslant n$, а максимум берется по наборам $\bar{m} \in\{0,1\}^{n},\|\bar{m}\|=\nu, \varepsilon$ зависит от $\mathbb{H}_{a}$.

Теперь применим предложение 4.1 к решетке $\mathrm{M}$, норме $\|\cdot\|_{A}$ и евклидовой метрике $|\cdot|_{Q}$. Отметим, что мы действуем в $\nu$-мерном пространстве $\mathscr{V}_{\nu}=\langle\mathrm{M}\rangle_{\mathbb{R}}$. Здесь единичным шаром нормы будет $\mathscr{W}_{\nu}\left(\|\cdot\|_{A}\right)=\mathscr{W}_{n} \cap \mathscr{V}_{\nu}$. Оценим его $Q$-евклидов объем с помощью неравенства

$$
\operatorname{Vol}_{\nu} \mathscr{W}_{\nu}\left(\|\cdot\|_{A}\right) \geqslant \operatorname{Vol}_{\nu}\left(\mathscr{S}_{R} \cap \mathscr{V}_{\nu}\right)=R^{\nu}(\pi / 2)^{\nu / 2} /(\nu / 2) !
$$

Тогда предложение 4.1 дает существование независимых векторов $\bar{Z}_{1}, \ldots, \bar{Z}_{\nu} \in$ М, удовлетворяюших неравенству

$$
\begin{aligned}
& \left\|\bar{Z}_{1}\right\|_{A} \ldots\left\|\bar{Z}_{\nu}\right\|_{A} \leqslant 2^{\nu} \frac{\mathrm{Vol}_{\nu} \mathrm{M}}{\operatorname{Vol}_{\nu} \mathscr{W}_{\nu}\left(\|\cdot\|_{A}\right)} \\
& \quad \leqslant 2^{\nu}\left(\frac{2 n}{\pi}\right)^{\nu / 2}\left(\frac{\nu}{2}\right) !\left(\begin{array}{c}
n \\
\nu
\end{array}\right)^{1 / 2} \gamma_{r} L_{0}^{\prime \varepsilon} \max \left\{\bar{A}^{\bar{m}} \bar{L}^{\prime \bar{m}}\right\} .
\end{aligned}
$$

Неравенства (19.7), (19.8) получаются отсюда соответственно с учетом вида группы $\mathbb{H}$. Если $r=\nu$, то мы имеем (19.7), а если $r=\nu+1$, то получаем (19.8). Это доказывает предложение 19.2. Отметим, что нам не пришлось пользоваться леммой Малера 4.2. 


\section{§20. Доказательство основной теоремы}

В предыдущих параграфах мы построили, в предположении о малости линейной формы от логарифмов (см. (12.2)), ненулевое решение системы $(9.9)$ для $(s, \nu)=$ $(S, n)$. Далее символ $\nu$ в этом контексте не используется. Система (9.9) эквивалентна системе (9.5), т.е. имеет вид (19.2) с многочленом $P=\mathscr{F}_{S}$ из (9.1).

Как уже отмечалось во введении, в отношении параметров $A_{j}$ в данной работе получены два усиления оценок линейных форм. Во-первых, сняты нижние ограничения на числа $A_{j}$, т.е. они теперь могут быть малыми (усиление работы [4], частично проведенное в [1]). И, во-вторых, множители $A_{j}$ удалены из параметра $C_{0}$ (сделано в [4] с помощью предложения 19.1, но не сделано в [1]). Это разноплановые усиления, поскольку наличие $A_{j}<1$ в $C_{0}$ только улучшило бы оценку $|\Lambda|$. Так что в этом случае целесообразнее было бы оставить в $C_{0}$ такие множители, но для простоты и единообразия изложения мы этого не делаем.

Все же возможная малость $\Omega$ создает в применении предложения 19.2 некоторые проблемы, поэтому, прежде чем его исследовать, рассмотрим более простой случай, а именно когда

$$
C_{0} \omega<e^{-3} n^{2} c_{1} / 3
$$

В этом случае с учетом определения параметра $S$ и оценки (5.3) получаем, что суммирование в $\mathscr{F}_{S}$ по $l_{j}, 1 \leqslant j \leqslant n$, исчезает, поскольку диапазон для изменения этих индексов становится $<1 / N$. Таким образом, $\mathscr{F}_{S}$ становится просто многочленом от $Y_{0}$.

Из неравенства (11.5) видно, что нулей $\mathscr{F}_{S}$ с учетом кратностей не меньше чем

$$
\left|\mathscr{X}_{S, n}\right|\left(T_{S}+1\right) \geqslant 2^{n+2} G>L_{0},
$$

что противоречит условию $\mathscr{F}_{S} \neq 0$ и тем самым доказывает теорему в случае малого $\omega$. Так что далее считаем

$$
C_{0} \omega \geqslant e^{-3} n^{2} c_{1} / 3
$$

В случае выполнения (20.1) можно увеличить нижнюю оценку для $X_{S}$, а именно

$$
X_{S} \geqslant 0.5 e^{-3} n^{2} c_{1} \xi D W_{0} .
$$

При $T_{S}=0$ неравенство получается непосредственно из (11.3) и (20.1), а при $T_{S} \geqslant 1$ - из определения числа $S$, поскольку

$$
T_{S}+1 \leqslant 2^{1-S} C_{0} \xi \omega / c_{1}
$$

Как отмечалось в $\S 9$, линейной заменой переменных можно сделать показатели целыми и неотрицательными, а нули сместить в неотрищательную область и придать им вид $x=0, \ldots, X^{\prime}$.

Займемся теперь применением предложения 19.2. Для этого установим соответствие параметров $\S 19$ с ранее введенными переменными. Поскольку в функции $\mathscr{F}$ показатели были дробные со знаменателем $N$, то надо определить числа $\vartheta_{j}$ из 
равенств $\ln \vartheta_{j}=\frac{1}{N} \ln \alpha_{j}$ (числа остаются алгебраическими, а степень поля в предложении 19.2 не имеет значения). Теперь положим

$$
\begin{aligned}
X^{\prime} & =\left|\mathscr{X}_{S, n}\right|-1 \geqslant 2^{n+1}\left\lceil 0.5 e^{-3} n^{2} \xi c_{1} D W_{0}\right\rceil, \\
X_{\nu}^{\prime} & =\left[\frac{X^{\prime}}{n+1}\right], \quad 0 \leqslant \nu \leqslant n, \\
M^{\prime} & =M_{S, n} \geqslant \frac{\varepsilon_{0} \xi C_{0} \omega}{1+\varepsilon_{0}}=\frac{6 n^{3} C_{0} \omega(n+1)}{c_{1}}, \\
M_{\nu}^{\prime} & =\left[\frac{M^{\prime}}{n+1}\right], \quad 0 \leqslant \nu \leqslant n, \\
L_{0}^{\prime} & =L_{0}, \quad L_{j}^{\prime}=\left[2^{-S} \frac{N L}{A_{j}}\right], \quad 1 \leqslant j \leqslant n,
\end{aligned}
$$

и оценим введенные выше параметры.

С учетом порядка величин $c_{1}$ и $\varepsilon_{0}$ имеем

$$
\begin{aligned}
X_{\nu}^{\prime} \geqslant \frac{X^{\prime}}{n+1}-1 & \geqslant \frac{1}{n+1} 2^{n} e^{-3} n^{2} \xi c_{1} D W_{0}-1 \geqslant 2^{n+1} e^{-3} n^{2} \xi D W_{0}, \\
M_{\nu}^{\prime}+1 & \geqslant \frac{6 n^{3} C_{0} \omega}{c_{1}}, \quad 0 \leqslant \nu \leqslant n .
\end{aligned}
$$

Из формулы для $S$ и неравенства (5.3) следует, что

$$
L_{j}^{\prime} \leqslant 2^{-S} \frac{N L}{A_{j}} \leqslant \frac{3 e^{3}}{n^{2} c_{1}} C_{0} \omega \min \left\{1, \frac{1}{1.5 A_{j}}\right\}, \quad 1 \leqslant j \leqslant n,
$$

откуда, в частности, $L_{j}^{\prime} \leqslant M_{j}^{\prime}$.

Теперь проверим выполнение других условий предложения 19.1.

Поскольку $W_{0}>C_{0} / 2$ и $\varepsilon_{0}$ достаточно мало, то $L_{0}^{\prime}>M^{\prime}+1$ и $L_{0}^{\prime} \geqslant L_{j}^{\prime}$, $j \geqslant 1$. Для упрощения проверки условия (19.1) упорядочим степени $L_{j}^{\prime}$ каким-либо образом, например будем считать, что

$$
L_{0}^{\prime} \geqslant L_{1}^{\prime} \geqslant \cdots \geqslant L_{n}^{\prime}
$$

Это позволяет считать в (19.1), что $m_{0}=\cdots=m_{\nu}=1, m_{\nu+1}=\cdots=m_{n}=0$.

Отметим также, что при $L_{j}^{\prime}=0$ можно считать $m_{j}=0$, иначе неравенство (19.1) становится тривиальным. Теперь начнем проверку выполнения условия (19.1). В случае $\nu<n$ достаточно проверить неравенство

$$
\left(X_{\nu}^{\prime}+1\right)\left(\frac{M^{\prime}}{n+1}\right)^{\nu+1}>((\nu+1) !)^{2} L_{0}^{\prime} L_{1}^{\prime} \ldots L_{\nu}^{\prime}
$$

или после подстановки оценок для $X_{\nu}^{\prime}, M^{\prime}, L_{0}, L_{j}^{\prime}$ и замены $(\nu+1)$ ! на выражение $e^{-\nu}(\nu+1)^{\nu+1.5}$ неравенство

$$
\frac{2^{n+1} n^{2} \xi D W_{0}}{e^{3}}\left(\frac{6 n^{3} C_{0} \omega}{c_{1}}\right)^{\nu+1} \geqslant e^{-2 \nu}(\nu+1)^{3+2 \nu} W_{0} \xi \omega\left(\frac{3 e^{3} C_{0} \omega}{n^{2} c_{1}}\right)^{\nu} .
$$


После сокрашения одинаковых множителей и логарифмирования остается проверить неравенство

$$
\ln \left(\frac{3 \cdot 2^{n+2} n^{5} D C_{0}}{e^{3}(\nu+1)^{3} c_{1}}\right)+\nu \ln \left(\frac{6 n^{5}}{3 e(\nu+1)^{2}}\right) \geqslant 0
$$

Левая сторона выпукла вверх по $\nu$, поэтому неравенство достаточно проверить при $\nu=0$ и $\nu=n-1$, что элементарно.

При $\nu=n$ с учетом множителей $A_{j}$ в $(20.2)$ необходимо проверить, что

$$
\frac{2^{n+1} n^{2} \xi D W_{0}}{e^{3}}\left(\frac{6 n^{3} C_{0} \omega}{c_{1}}\right)^{\nu} \geqslant e^{2-2 n}(n+1)^{2} n^{2 n} W_{0} \xi \omega\left(\frac{3 e^{3} C_{0} \omega}{n^{2} c_{1}}\right)^{n} \frac{1}{1.5^{n} \Omega} .
$$

Тогда после подстановки формулы для $\omega$ и сокращения одинаковых членов получаем

$$
\frac{2 n^{2}}{e^{5}(n+1)^{2}}\left(\frac{6 n^{3}}{e}\right)^{n} \geqslant \frac{\gamma}{N}
$$

Последнее неравенство также верно ввиду того, что $\gamma \leqslant N$ и $n \geqslant 2$.

Для проверки условия (19.4) отметим, что

$$
X^{\prime} \leqslant 2^{n+1} e^{-3} n^{3} \xi c_{1} D W_{0} \leqslant e^{C_{0}} e^{W_{0} / e} \leqslant e^{2 \varepsilon_{1} G},
$$

и в предположении (12.2) выполнено

$$
\left|b_{1} \ln \vartheta_{1}+\cdots+b_{n} \ln \vartheta_{n}\right|=\frac{1}{N}|\Lambda|<e^{-4 \varepsilon_{1} G}<\frac{2 \pi}{X^{\prime}+1} .
$$

Так же легко проверяются условия (19.5), (19.6).

Таким образом, условия $\S 19$ выполнены и можно применить предложение 19.2. Если мы покажем, что из любого неравенства (19.7), (19.8) следует (7.3), то получим противоречие с индуктивным предположением $\S 7$ и тем самым докажем теорему 2.1 .

Поскольку числа $A_{j} L_{j}^{\prime}, j \geqslant 1$, оцениваются одинаково и не превосходят $L_{0}^{\prime}$, то мы можем считать, что в (19.7), (19.8)

$$
m_{1}=\cdots=m_{\nu}=1, \quad m_{\nu+1}=\cdots=m_{n}=0 .
$$

Далее, поскольку все $X_{\nu}^{\prime}$ равны и равны $M_{\nu}^{\prime}$ и мы имеем

$$
W_{0}\left(M_{\nu}^{\prime}+\nu\right) / \nu \leqslant(\nu+1) C_{0} L_{0}^{\prime}
$$

то из неравенств $(19.7),(19.8)$ достаточно рассмотреть только $(19.8)$, или

$$
\frac{2^{n+1} n^{2} \xi D W_{0}}{e^{3}}\left(\frac{6 n^{3} C_{0} \omega}{c_{1}}\right)^{\nu} A_{1}^{\prime} \ldots A_{\nu}^{\prime} \geqslant(\nu+1) ! \mathscr{C}(\nu) W_{0} \xi \omega\left(\frac{2 e^{3} C_{0} \omega}{n^{2} c_{1}}\right)^{\nu} .
$$


После сокращений одинаковых членов, подстановки (19.9) для $\mathscr{C}(\nu)$ с заменой $\left(\begin{array}{l}n \\ \nu\end{array}\right) \leqslant n^{\nu} / \nu !$ и $x ! \leqslant e \nu^{\nu+1 / 2} e^{-\nu}$ получаем

$$
A_{1}^{\prime} \ldots A_{\nu}^{\prime} \leqslant \frac{\gamma}{N} g(\nu) \Omega, \quad g(\nu)=\frac{e^{11 / 2}(\nu+1) \nu^{5 / 4}}{2^{n+3 / 2} n^{2}}\left(\frac{4 e^{2} \nu^{4}}{9 \pi n^{8}}\right)^{\nu / 2} .
$$

При этом легко проверяется, что

$$
g(\nu) \leqslant \frac{1}{2}\left(4 e^{2}\right)^{n-\nu}, \quad 1 \leqslant \nu \leqslant n .
$$

Но тогда из (20.3) следует условие (7.3). Это противоречит индуктивной гипотезе и доказывает теорему.

Отметим еше один элемент новизны в доказательстве теоремы 2.1. Как было видно, числа $\vartheta_{1}, \ldots, \vartheta_{n}$ из леммы 18.1 использовались только для обоснования деления аргумента. В остальном вычисления проводились непосредственно с числами $\alpha_{1}, \ldots, \alpha_{n}$. В предшествующих работах в той или иной форме осуществлялся переход с помощью леммы 4.2 к $\vartheta_{j}$, что требовало пересчета высот и коэффициентов линейной формы. Именно на этом этапе и появлялся множитель $n !$, а также происходил переход от $B$ из (1.3) к $B^{*}$ из (1.4).

\section{§21. Доказательство следствий}

Докажем теорему 2.2 путем сведения ее к теореме 2.1 за счет исключения зависимых логарифмов. Без ограничения общности можно считать, что $r$ - максимальное число независимых логарифмов.

При $r=1$ с некоторым $\alpha \in \mathbb{K}^{*}$ выполнено

$$
\ln \alpha_{j}= \pm m_{j} \ln \alpha, \quad m_{j} \in \mathbb{N}, \quad 1 \leqslant j \leqslant n,
$$

при этом $A=H_{\varkappa}(\alpha) \leqslant \min \left\{A_{j}: 1 \leqslant j \leqslant n\right\}$ и $|\Lambda| \geqslant|\ln \alpha|$. Здесь оценка получается сразу из неравенства Лиувилля, как в $\S 6$,

$$
|\Lambda| \geqslant \exp (-D \ln 2-A)
$$

Так что далее считаем $n \geqslant r>1$.

Разберем теперь случай $r=n, b_{n}=0$. Пусть, например, $b_{n-1} \neq 0$. Здесь можно индуктивно применить оценку (2.3) при $n-1$ :

$$
\begin{aligned}
-\ln |\Lambda| & \leqslant C(n-1) C_{0} D^{2} A_{1} \ldots A_{n-1} \ln \left(1.5 e B_{n-1} D \ln (e D)\right), \\
B_{n-1} & =\max \left\{\left|b_{j}\right| A_{j} / A_{n-1}: 1 \leqslant j \leqslant n-1\right\} \geqslant 1
\end{aligned}
$$

(так как при $j=n-1$ имеем $\left|b_{n-1}\right| \geqslant 1$ ).

Теперь, оценивая в $B_{n-1}$ компоненту $1 / A_{n-1}$ с помошью (5.3) и переходя к $B$, получаем

$$
-\ln |\Lambda| \leqslant C(n-1) C_{0} D^{2} A_{1} \ldots A_{n-1} \ln \left((1.5 D \ln (e D))^{2} e B A_{n}\right) .
$$


Вопрос состоит в том, можно ли добавить в правую часть (21.1) множитель $x=A_{n} \geqslant 0.16$ и разделить выражение под знаком логарифма на $x$. Тогда в правой части (21.1) будет находиться функция вида $f(x)=a x \ln (b / x)$, возрастающая на интервале $1 \leqslant x \leqslant b / e$ (включающем $A_{n}$ ), а при $x=1$ мы просто получим (21.1). Поэтому при $A_{n} \geqslant 1$ оценка (2.5) получается из (21.1) за счет мажорирования логарифма величиной $2 W_{0}$ и перехода к $C(n)>4 e^{2} C(n-1)$.

При $0.16 \leqslant A_{n} \leqslant 1$ мы, заменяя в $(21.1)$ величину $A_{n}$ под знаком логарифма на 1 и добавляя лишний множитель $A_{n}$ при переходе к $C(n)$, тоже получаем $(2.5)$, что полностью доказьвает случай $b_{n}=0$.

Пусть далее $1<r<n$. Мы можем также считать $b_{i} \neq 0, i>r$, иначе нулевые слагаемые можно исключить из линейной формы, а из оценки - множители $A_{i} \geqslant 0.16$, что компенсируется улучшением оценки при переходе к $C\left(n^{\prime}\right)$ с меньшим числом слагаемых $n^{\prime}$. Случай $b_{n}=0$ здесь аналогичен ситуации, когда $r=n$.

Кроме того, с учетом (2.4) для зависимых логарифмов получаем неравенство

$$
A_{1} \ldots A_{n} \xi_{0}(r) 0.16^{r-n} D \ln \left(e^{n+4} D\right) \geqslant 1
$$

и аналогичные оценки для меньшего числа сомножителей.

Предварительная часть доказательства проводится, как в $\S 6$, только с использованием (21.2) вместо (5.1), (5.2). Мы также можем считать вьполненными условия (6.1)-(6.4) с заменой (ввиду (21.2)) условия (6.2) на условие

$$
B \geqslant 0.99 \frac{1}{n \xi_{0}(r)} 0.16^{n-r} C(n) C_{0} D,
$$

или, огрубляя с учетом $r>1$, на условие

$$
B \geqslant B_{0}=0.16^{n} C(n) C_{0} D / n .
$$

Итак, пусть имеются целочисленные соотношения вида

$$
\Lambda_{i}=x_{i, 1} \ln \alpha_{1}+\cdots+x_{i, r} \ln \alpha_{r}-x_{i, i} \ln \alpha_{i}=0, \quad x_{i, i} \neq 0, \quad r<i \leqslant n .
$$

Однако их коэффищиенты могут быть не столь малыми, как требуется. Поэтому мы возьмем независимые целочисленные соотношения, удовлетворяюшие условиям леммы 4.3:

$$
\Lambda_{i}^{\prime}=m_{i, 1} \ln \alpha_{1}+\cdots+m_{i, n} \ln \alpha_{n}=0, \quad 1 \leqslant i \leqslant n-r .
$$

Поскольку линейные формы $\Lambda_{i}^{\prime}$ имеют максимальный ранг, то через них выражаются и $\Lambda_{i}$. Отсюда следует, что у матрицы коэффициентов $\left(m_{i, j}\right)$ минор с индексами $j>r$ ненулевой.

Теперь построим новую линейную форму

$$
\begin{aligned}
\Lambda^{\prime} & =z_{0} \Lambda=z_{0} \Lambda-z_{1} \Lambda_{1}^{\prime}-\cdots-z_{n-r} \Lambda_{n-r}^{\prime} \\
& =b_{1}^{\prime} \ln \alpha_{1}+\cdots+b_{n}^{\prime} \ln \alpha_{n} \neq 0, \quad z_{0}, \ldots, z_{n-r} \in \mathbb{Z}, \quad z_{0} \neq 0,
\end{aligned}
$$


с условием $b_{r+1}^{\prime}=\cdots=b_{n}^{\prime}=0$. Для этого достаточно положить согласно правилу Крамера $z_{0}=\Delta$, где $\Delta$ - вышеупомянутый ненулевой минор, а $z_{i}$ получается заменой в миноре $i$-й строки на строку коэффициентов $b_{j}, j>r$.

Поскольку $\Lambda^{\prime} \neq 0$, то без ограничения общности можно считать, что $b_{r}^{\prime} \neq 0$. Положим

$$
\begin{aligned}
B^{\prime}=\left|\mathbf{b}^{\prime}\right|_{A} / A_{r} & =\max \left\{\left|b_{j}\right| A_{j} / A_{r}: 1 \leqslant j \leqslant r\right\} \geqslant 1, \\
W_{0}^{\prime} & =\ln \left(1.5 e B^{\prime} D \ln (e D)\right) .
\end{aligned}
$$

ЛЕмма 21.1. С учетом принятых обозначений и допущений (2.4), (6.1) при $1<r<n$ выполняется

$$
W_{0}^{\prime} \leqslant \frac{3}{4} n \ln \left(1.5 e B A_{n} D \ln (e D)\right) .
$$

ДокАЗАТЕЛЬСтво. Воспользуемся неравенством (4.1), леммой 5.3, а также формулой Стирлинга и неравенством $\left(\begin{array}{c}n \\ r\end{array}\right) \leqslant n^{r} / r !$ :

$$
\begin{aligned}
\prod_{i=1}^{n-r}\left|\mathbf{m}_{i}\right|_{A} & \leqslant 2.2 \cdot 3^{r / 2}\left(\begin{array}{c}
n \\
r
\end{array}\right)^{1 / 2}\left(\frac{9}{8} D^{3 / 2} \ln (e D)\right)^{r} \frac{(r / 2) !}{\pi^{r / 2}} A_{1} \ldots A_{n} \\
& \leqslant 4 \sqrt{n}(n R)^{r / 2} A_{1} \ldots A_{n}, \quad R=D^{3} \ln ^{2}(e D) .
\end{aligned}
$$

Для оценки коэффициентов $z_{i}$ из представления формы $\Lambda^{\prime}$ применим лемму 4.4 c тах-нормой для последних $n-r$ коэффищиентов. Тогда

$$
\left|z_{0}\right| \leqslant n^{(n-r) / 2} \frac{\left|\mathbf{m}_{1}\right|_{A} \ldots\left|\mathbf{m}_{n-r}\right|_{A}}{A_{r+1} \ldots A_{n}}
$$

откуда

$$
\left|z_{0} b_{j}\right| A_{j} \leqslant B A_{n} n^{(n-r) / 2} \frac{\left|\mathbf{m}_{1}\right| A \ldots\left|\mathbf{m}_{n-r}\right|_{A}}{A_{r+1} \ldots A_{n}} .
$$

Так же оцениваются величины $\left|z_{i} m_{i, j}\right| A_{j}, 1 \leqslant i \leqslant n-r ; 1 \leqslant j \leqslant r$. Поэтому

$$
B^{\prime}=\left|\mathbf{b}^{\prime}\right|_{A} / A_{r} \leqslant B A_{n} n^{1+(n-r) / 2} \frac{\left|\mathbf{m}_{1}\right|_{A} \ldots\left|\mathbf{m}_{n-r}\right|_{A}}{A_{r} A_{r+1} \ldots A_{n}} .
$$

Теперь применим неравенство (21.5). При этом один множитель $A_{n}$ сократится, для множителя $A_{1} \ldots A_{n-1}$ в числителе применим оценку (6.4), для $A_{r}$ в знаменателе применим (5.3), а для остальных $A_{j}$ в знаменателе воспользуемся оценкой $A_{j} \geqslant 0.16(r<j<n)$. Тогда

$$
\begin{aligned}
B^{\prime} & \leqslant B A_{n} n^{1+(n-r) / 2} 4 \sqrt{n}(n R)^{r / 2} \frac{A_{1} \ldots A_{n-1}}{A_{r} \ldots A_{n-1}} \\
& \leqslant 4 B A_{n} n^{(n+3) / 2} R^{(n-1) / 2} 0.16^{3-n} \frac{1.5 D \ln (e D) B n}{0.99 C(n) D^{2} C_{0} W_{0}} \\
& =f_{0}\left(B, A_{n}, D, n\right) .
\end{aligned}
$$


Для завершения доказательства неравенства (21.4) покажем, что

$$
f_{1}\left(B, A_{n}, D, n\right)=\left(1.5 e B A_{n} D \ln (e D)\right)^{3 n / 4} \geqslant f_{0} .
$$

Заметим, что в $f_{0}$ множитель $A_{n}$ входит в первой степени, а в $f_{1}-$ в степени $3 n / 4 \geqslant 2$. Поэтому для проверки неравенства $f_{1}>f_{0}$ следует взять минимально возможное $A_{n}=0.16$. Аналогично для $B$ тоже надо взять минимально возможное значение, определяемое из условия (21.3), и подставить $B_{0}$ в $W_{0}$. После такой подстановки зависимость от $D$ в $f_{1} / f_{0}$ станет равной

$$
\frac{\left(D^{2} C_{0} \ln (e D)\right)^{3 n / 4} D^{2} C_{0} W_{0}}{\left(C_{0} D\right)^{2} D \ln (e D)\left(D^{3} \ln ^{2}(e D)\right)^{(n-1) / 2}},
$$

и с учетом $C_{0}>\ln (e D), n \geqslant 3$, вышеприведенное выражение растет с ростом $D$. Поэтому далее можно считать $D=1$.

После указанных подстановок $B, A_{n}, D$ получаем

$$
\begin{aligned}
f_{1} / f_{0} \geqslant & \left(1.5 e 0.16^{n+1} C(n) C_{0} / n\right)^{3 n / 4-2} \\
& \times 0.99 \frac{1}{4} n^{-(n+5) / 2} 0.16^{n-2} C(n) C_{0} W_{0} \\
\geqslant & \left(0.5 n^{-2} 0.16^{n} C(n) C_{0}\right)^{3 n / 4-2} \\
& \times 0.24 n^{n / 4-9 / 2} 0.16^{n-2} C(n) C_{0} W_{0} \geqslant 1,
\end{aligned}
$$

где каждая строка $\geqslant 1$ за счет роста $C(n)$. Лемма 21.1 доказана.

$\mathrm{B} \Lambda^{\prime}$ появился дополнительный множитель $z_{0}$, оцениваемый с помощью (21.5), (21.6) аналогично оценке $B^{\prime}$ :

$$
\begin{aligned}
\left|z_{0}\right| & \leqslant n^{(n-r) / 2} 4 \sqrt{n}(n R)^{r / 2} \frac{A_{1} \ldots A_{n}}{A_{r+1} \ldots A_{n}} \\
& \leqslant 4 \sqrt{n} n^{n / 2}\left(D^{3} \ln ^{2}(e D)\right)^{r / 2} 0.16^{r-n} A_{1} \ldots A_{n} \\
& \leqslant e^{2(n-1) C_{0}} e^{\Omega / e} \leqslant e^{2 n \varepsilon_{1} G}
\end{aligned}
$$

$\left(\varepsilon_{1}, G\right.$ из $\left.\S 12\right)$.

Применим теперь к $\Lambda^{\prime}$ теорему 2.1 с заменой $n$ на $r$. Тогда с учетом (21.4) и $A_{j} \geqslant 0.16$ (при $j>r$ ) получаем

$$
\begin{aligned}
-\ln |\Lambda| & \leqslant \ln \left|z_{0}\right|-\ln \left|\Lambda^{\prime}\right| \leqslant 2 n \varepsilon_{1} G+C(r) D^{2} A_{1} \ldots A_{r} C_{0} W_{0}^{\prime} \\
& \leqslant 2 n \varepsilon_{1} G+\frac{3}{4} n C(n-1) D^{2} A_{1} \ldots A_{n-1} C_{0} \ln \left(1.5 e B A_{n} D \ln (e D)\right) \\
& \leqslant \frac{1}{6} n C(n) D^{2} A_{1} \ldots A_{n} C_{0} \ln (1.5 e B D \ln (e D)),
\end{aligned}
$$

что доказывает (2.5) в случае зависимых логарифмов. Здесь мы поступили с коэффициентом $A_{n}$ так, как в случае $r=n, b_{n}=0$, а также применили неравенство

$$
2 n \varepsilon_{1} C(n)+\frac{3}{4 \cdot 0.16} n C(n-1) \leqslant \frac{1}{6} n C(n) .
$$


Для доказательства следствия 2.3 заметим, что ввиду (21.3) можно считать $W_{0} \leqslant 2 \ln (e B)$, поэтому достаточно проверить для $n \geqslant 2, \varkappa \leqslant 2$ неравенство

$$
2 C(n, \varkappa) C_{0}(n, D) \min \{1, n / 6\} \leqslant C_{1}(n, \varkappa) \ln (e D) .
$$

С учетом очевидного неравенства $C_{0}(n, D) \leqslant C_{0}(n, 1) \ln (e D)$ для проверки (21.7) достаточно рассмотреть $D=1$. Далее, главная зависимость от $n$ в $C(n)$ имеет вид $\left(4 e^{2}\right)^{n}$, при этом $4 e^{2}=29.5 \ldots<30$. Значит, $(21.7)$ выполняется для больших $n$, а при малых $n$ оценка (21.7) проверяется непосредственным вычислением. Это доказывает (2.6).

Чтобы заменить $B$ на $B^{*}$ из (1.4), достаточно считать (меняя при необходимости местами слагаемые), что $A_{n}$ - максимальное из $A_{j}$ (если для всех $A_{j}$ имеем $(2.4)$, то они становятся равноправными). Тогда $B \leqslant B^{*}$, откуда получаем последнее утверждение следствия.

\section{§22. Дополнение}

В данном параграфе доказываются результаты геометрического характера, относящиеся преимущественно к предшествующим работам автора.

Оценка объема усеченного множества $\mathscr{W}_{0}$ из $§ 10$ была получена в [1] с помощью теоремы Брунна-Минковского. Также эта теорема была использована в работах автора [5], [8]. Поскольку там применение теоремы было не прямым и не столь очевидным, мы считаем полезным явно сфформулировать и доказать соответствующее утверждение.

ПРЕДЛОЖЕНИЕ 22.1 (теорема Брунна-Минковского [21, теорема 21.1]). Пусть в $\mathbb{R}^{d}$ даны компактные выпуклые тела $K_{1}, K_{2}$. Тогда для любых $\lambda_{1}, \lambda_{2}>0$, $\left(\lambda_{1}+\lambda_{2}=1\right)$ справедливо неравенство

$$
\mathrm{Vol}_{d}^{1 / d}\left(\lambda_{1} K_{1}+\lambda_{2} K_{2}\right) \geqslant \lambda_{1} \operatorname{Vol}_{d}^{1 / d} K_{1}+\lambda_{2} \operatorname{Vol}_{d}^{1 / d} K_{2} .
$$

Мы использовали следующий результат.

Лемма 22.2. Пусть в $\mathbb{R}^{n}$ даны $d$-мерное подпространство $\mathscr{V} \quad(1 \leqslant d<n)$ и компактное выпуклое тело $\mathscr{W}, \mathscr{V} \perp-$ ортогональное дополнение $\mathscr{V}, f(\mathbf{v})=$ $\operatorname{Vol}_{d}^{1 / d}(\mathscr{W} \cap(\mathbf{v}+\mathscr{V})), \quad \mathbf{v} \in \mathscr{V}^{\perp}$. Тогда $f(\mathbf{v})$ - вылуклая вверх функция на множестве 'W", являющемся проекцией $\mathscr{W}$ на $\mathscr{V} \perp$.

Если $\mathscr{W}$ - $\mathbf{0}$-симметричное выпуклое тело, то для любого $\mathbf{v} \in \mathscr{V}^{\perp}, \mathbf{v} \neq 0$, функиия $f(t \mathbf{v})$ убьввает при $t \geqslant 0$.

ДокАЗАТЕЛЬСТво. Пусть $K(\mathbf{v})=\mathscr{W} \cap(\mathbf{v}+\mathscr{V}), \mathbf{v} \in \mathscr{V} \perp, K^{\prime}$ - проекция $K$ на $\mathscr{V}$. Поскольку проекция сохраняет выпуклость, линейные соотношения между множествами и $d$-мерные объемы тел, расположенных вдоль $\mathscr{V}$, то по теореме 22.1 для $\lambda_{1}, \lambda_{2}>0\left(\lambda_{1}+\lambda_{2}=1\right)$ имеем

$$
\begin{aligned}
\lambda_{1} f & \left(\mathbf{v}_{1}\right)+\lambda_{2} f\left(\mathbf{v}_{2}\right)=\lambda_{1} \operatorname{Vol}_{d}^{1 / d} K\left(\mathbf{v}_{1}\right)+\lambda_{2} \operatorname{Vol}_{d}^{1 / d} K\left(\mathbf{v}_{2}\right) \\
& =\lambda_{1} \operatorname{Vol}_{d}^{1 / d} K^{\prime}\left(\mathbf{v}_{1}\right)+\lambda_{2} \operatorname{Vol}_{d}^{1 / d} K^{\prime}\left(\mathbf{v}_{2}\right) \\
& \leqslant \operatorname{Vol}_{d}^{1 / d}\left(\lambda_{1} K^{\prime}\left(\mathbf{v}_{1}\right)+\lambda_{2} K^{\prime}\left(\mathbf{v}_{2}\right)\right)=\operatorname{Vol}_{d}^{1 / d}\left(\lambda_{1} K\left(\mathbf{v}_{1}\right)+\lambda_{2} K\left(\mathbf{v}_{2}\right)\right) \\
& \leqslant \operatorname{Vol}_{d}^{1 / d} K\left(\lambda_{1} \mathbf{v}_{1}+\lambda_{2} \mathbf{v}_{2}\right)=f\left(\lambda_{1} \mathbf{v}_{1}+\lambda_{2} \mathbf{v}_{2}\right) .
\end{aligned}
$$


Последнее неравенство выполняется в силу выпуклости $\mathfrak{W}$.

Для симметричного тела $\mathscr{W}$ функция $f(t \mathbf{v})$ выпукла вверх в области $f(t \mathbf{v})>0$ и четна по $t$, откуда следует второе утверждение леммы.

Использование леммы 22.2 в $\S 10$ состояло в том, что при отсечении краев у выпуклого тела $\mathscr{W}$ остается тело $\mathscr{W}_{0}$ достаточно большого объема.

ДокАЗАТЕЛЬСТВо ЛЕммы 5.3. В общем случае из (5.4) имеем

$$
\|\mathbf{v}\|_{2}^{2} \leqslant \sum^{(*)} y_{\sigma}^{2}+\left(\sum^{(+)} x_{\sigma}\right)^{2}+\left(\sum^{(-)} x_{\sigma}\right)^{2} \leqslant(k+2)\|\mathbf{v}\|^{2}
$$

(в суммах $\sum^{( \pm)}$сгруппированы слагаемые, имеющие одинаковый знак). Поэтому если $\left\|\mathbf{e}_{j}\right\| \leqslant 1,1 \leqslant j \leqslant r$, то $\left\|\mathbf{e}_{j}\right\|_{2} \leqslant(k+2)^{1 / 2}, 1 \leqslant j \leqslant r$, и объем построенного на них параллелепипеда не больше $(k+2)^{r / 2}$. Это доказывает оценку для шара $\mathscr{B}(\|\cdot\|, r)$.

Для оценки объема решеток достаточно указать в пространстве $\mathscr{V}_{r}=\langle\mathrm{M}\rangle_{\mathbb{R}}$ тело $\mathscr{S}_{r}$, удовлетворяюшее условию $2 \mathscr{S}_{r} \cap \mathrm{M}=\{\mathbf{0}\}$. Тогда по теореме Минковского $\operatorname{Vol}_{r} \mathrm{M} \geqslant \operatorname{Vol}_{r} \mathscr{S}_{r}$.

Возьмем в случае $\mathbb{Q}$ тело

$$
\mathscr{S}=\left\{\mathbf{v} \in \mathscr{E}_{1}\left(\mathbb{Q}^{+}\right):\left|x_{\sigma}\right|<\frac{1}{2} \ln p_{\sigma}, \quad \sigma>1\right\},
$$

а если в $\mathbb{Q}$ можно брать отрицательные числа, то добавим условие

$$
\left|y_{1}\right|<\frac{1}{2} \pi
$$

Тогда при сечении $\mathscr{S}$ каким-либо $r$-мерным подпространством получается тело объема не менее $P_{1} \ldots P_{r}$ (согласно лемме Ваалера о сечениях куба [22] случай параллелепипеда см. в [8, лемма 7] или [23, оценка (3.2)]). Кроме того, ясно, что $\mathrm{M}_{\varkappa}(\mathbb{Q}) \cap 2 \mathscr{S}=\{\mathbf{0}\}$, откуда следует $(5.6)$.

В случае $D>1$ рассмотрим тело

$$
\mathscr{S}=\left\{\mathbf{v} \in \mathscr{E}_{\varkappa}(\mathbb{K}):\|\mathbf{v}\|_{2}<R\right\}, \quad R=\left(\frac{9}{8} D^{3 / 2} \ln (e D)\right)^{-1},
$$

и покажем, что $\mathrm{M}_{\varkappa}(\mathbb{K}) \cap 2 \mathscr{S}=\{\mathbf{0}\}$.

Пусть $\mathbf{x}=\mathbf{x}(\alpha)$. Если $\alpha$ не единица, то для какой-либо $p$-адической координаты имеем $\left|x_{\sigma}\right| \geqslant \ln 2>2 R$, поэтому $\mathbf{x} \notin 2 \mathscr{S}$. Так что далее считаем $\alpha$ единицей, т.е. $x_{\sigma}=0, \sigma>D$.

Для $D=2,3$ малые алгебраические числа известны. Здесь, если $\alpha$ не корень из 1 , то при $D=2$ имеем $\max \left|x_{\sigma}\right|>0.48$ (минимум достигается для корня уравнения $x^{2}-x-1=0$ ), а при $D=3$ имеем $\max \left|x_{\sigma}\right|>0.28$ (минимум достигается для корня уравнения $x^{3}-x-1=0$ ); в обоих случаях $\max \left|x_{\sigma}\right|>2 R$. Для $\alpha \neq 1$, являющегося корнем из 1 , здесь имеем $|\ln \alpha| \geqslant \pi / 3$. Во всех случаях получаем $\|\mathbf{x}(\alpha)\|_{2}>2 R$ и $\mathrm{M}_{\varkappa}(\mathbb{Q}) \cap 2 \mathscr{S}=\{\mathbf{0}\}$.

Если $D \geqslant 4$ и $\mathbf{x}(\alpha) \in 2 \mathscr{S}$, то $x_{1}^{2}+\cdots+x_{D}^{2}<(2 R)^{2}$, значит, $\left|x_{1}\right|+\cdots$ $\cdots+\left|x_{D}\right|<2 R \sqrt{D}$ и $D \mathrm{~h}(\alpha)<R \sqrt{D}$. Кроме того, $|\ln \alpha|=\left|z_{1}\right|<2 R \leqslant R \sqrt{D}$, значит, $\mathrm{H}_{\varkappa}(\alpha)<R \sqrt{D}$ и согласно предложению $5.2 \alpha=1$.

Теперь, рассматривая $\mathscr{S}_{r}=\mathscr{S} \cap\langle\mathrm{M}\rangle_{\mathbb{R}}$, получаем шар радиуса $R$, объем которого представлен правой частью неравенства (5.5). 
ПРимечАниЕ. Лемма 5.3 дает рабочую формулу для оценки с помощью леммы 4.2 коэффициентов в соотношениях между логарифмами. Следует отметить, что в аналогичных оценках из [8] (теоремы 4 и 6), получаюшихся соединением леммы 4.2 и теоремы Минковского, был пропущен множитель, соответствующий объему единичного шара нормы. Теорема 5 из [8] верна, поскольку аналогична случаю $\mathbb{Q}^{+}$леммы 5.3, только нумеровать в ней следует простые идеалы поля, а не простые числа. Исправленная версия теоремы 4 из [8] указана в [23] (без явной рабочей формулы).

Отметим, что для доказательства леммы 5.3 мы взяли обычный шар, сечения которого также являются шарами, а формула для их объема известна. При доказательстве аналогичной леммы в работе [2] был фактически взят куб, вписанньй в наш шар, что привело к худшей оценке.

В заключение автор выражает благодарность профессору Ю.В. Нестеренко, сделавшему ряд ценных замечаний.

\section{Список литературы}

1. Матвеев E.M. Явная нижняя оценка однородной рациональной линейной формы от логарифмов алгебраических чисел // Изв. РАН. Сер. матем. 1998. Т. 62. № 4. С. 81-136.

2. Waldschmidt M. A lower bound for linear forms in logarithms // Acta Arithm. 1980. V. 37. P. 257-283.

3. Матвеев E. M. Оценка линейной формы от логарифмов алгебраических чисел: Тез. докл. всесоюзн. конф. "Теория трансцендентных чисел и ее приложения" (М., 2-4 февр. 1983 г.). М.: Изд-во МГУ, 1983.

4. Baker A., Wüstholz G. Logarithmic forms and group varieties // J. reine angew. Math. 1993. V. 442. Р. 19-62.

5. Матвеев E. M. О последовательных минимумах расширенной логарифмической высоты алгебраических чисел // Матем. сб. 1999. Т. 190. № 3. С. 89-108.

6. Feldman N. I. Approximations of number $\pi$ by algebraic numbers from special fields // J. Number Theory. 1977. V. 9. № 1. P. 48-60.

7. Касселс Дж. В. С. Введение в геометрию чисел. М.: Мир, 1965.

8. Матвеев E. М. О линейных и мультипликативных соотношениях // Матем. сб. 1993. T. 184. № 4. C. 23-40.

9. Боревич З. И., Шафаревич И. Р. Теория чисел. М.: Наука, 1972.

10. Ленг C. Основы диофантовой геометрии. М.: Мир, 1986.

11. Матвеев E. М. Об алгебраических числах малой логарифмической высоты // Диофантовы приближения: Сб. статей, посвященных памяти Н. И. Фельдмана / Ред. Ю.В. Нестеренко. М.: Изд-во МГУ, 1996. С. 90-98.

12. Гельфонд А.О., Фельдман Н.И. О нижних границах линейных форм от трех логарифмов алгебраических чисел // Вестн. МГУ. Сер. матем., механ. 1949. № 5. С. 13-16; см. также Гельфонд А. О. Избранные труды. М.: Наука, 1973. С. 155-158.

13. Матвеев E. M. Об арифметических свойствах значений обобщенных биномиальных многочленов // Матем. заметки. 1993. Т. 54. № 4. С. 76-81.

14. Baker A. A sharpening of the bounds for linear forms in logarithms. III // Acta Arithm. 1975. V. 27. P. 247-252.

15. Baker A., Stark H. M. On a fundamental inequality in number theory // Ann. of Math. 1971. V. 94. P. 190-199.

16. Bombieri E., Vaaler J. On Siegel's Lemma // Invent. Math. 1983. V. 73. P. 11-32; Addendum in: Invent. Math. 1984. V. 75. P. 377.

17. Yu Kunrui. $p$-adic logarithmic forms and group varieties. I // J. reine angew. Math. 1998. V. 502. P. 29-92. 
18. Waldschmidt $M$. Minorations de combinaisons linéaires de logarithmes de nombres algébriques // Canad. J. Math. 1993. V. 45. № 1. P. 176-224.

19. Wüstholz $G$. A new approach to Baker's theorem on linear forms in logarithms. III // New advances in transcendence theory / Ed. A. Baker. Cambridge: Cambridge Univ. Press, 1988. P. 399-410.

20. Лейхтвейс К. Выпуклые множества. М.: Наука, 1985.

21. Vaaler J. D. A geometric inequality with application to linear forms // Pacific. J. Math. 1979. V. 83. P. 543-553.

22. Bertrand D. Duality on tori and multiplicative dependence relations // J. Austral. Math. Soc. Ser. A. 1997. V. 62. P. 198-216.

23. Meyer M., Pajor A. Sections of the unit ball of $l_{p}^{n} / /$ J. Func. Anal. 1988. V. 80. P. 109-123.

Московский государственный текстильный

Поступило в редакцию

университет им. А.Н. Косыгина

31.VIII.1999

12.VII. 2000 